REVIEWS OF MODERN PHYSICS, VOLUME 88, OCTOBER-DECEMBER 2016

\title{
Active particles in complex and crowded environments
}

\author{
Clemens Bechinger \\ Physikalisches Institut, Universität Stuttgart, Pfaffenwaldring 57, 70569 Stuttgart, Germany \\ and Max-Planck-Institut für Intelligente Systeme, \\ Heisenbergstraße 3, 70569 Stuttgart, Germany \\ Roberto Di Leonardo \\ Dipartimento di Fisica, Università "Sapienza," I-00185, Roma, Italy \\ and NANOTEC-CNR Institute of Nanotechnology, \\ Soft and Living Matter Laboratory, I-00185 Roma, Italy \\ Hartmut Löwen \\ Institut für Theoretische Physik II: Weiche Materie, Heinrich-Heine-Universität Düsseldorf, \\ D-40225 Düsseldorf, Germany \\ Charles Reichhardt \\ Theoretical Division, Los Alamos National Laboratory, Los Alamos, New Mexico 87545, USA
}

Giorgio Volpe

Department of Chemistry, University College London, 20 Gordon Street, London WC1H OAJ, United Kingdom

\author{
Giovanni Volpe* \\ Department of Physics, University of Gothenburg, SE-41296 Gothenburg, Sweden \\ and Soft Matter Lab, Department of Physics, \\ and UNAM-National Nanotechnology Research Center, \\ Bilkent University, Ankara 06800, Turkey \\ (published 23 November 2016)
}

\begin{abstract}
Differently from passive Brownian particles, active particles, also known as self-propelled Brownian particles or microswimmers and nanoswimmers, are capable of taking up energy from their environment and converting it into directed motion. Because of this constant flow of energy, their behavior can be explained and understood only within the framework of nonequilibrium physics. In the biological realm, many cells perform directed motion, for example, as a way to browse for nutrients or to avoid toxins. Inspired by these motile microorganisms, researchers have been developing artificial particles that feature similar swimming behaviors based on different mechanisms. These man-made micromachines and nanomachines hold a great potential as autonomous agents for health care, sustainability, and security applications. With a focus on the basic physical features of the interactions of self-propelled Brownian particles with a crowded and complex environment, this comprehensive review will provide a guided tour through its basic principles, the development of artificial self-propelling microparticles and nanoparticles, and their application to the study of nonequilibrium phenomena, as well as the open challenges that the field is currently facing.
\end{abstract}

DOI: 10.1103/RevModPhys.88.045006

\section{CONTENTS}

I. Introduction

II. Noninteracting Active Particles in Homogenous Environments

A. Brownian motion versus active Brownian motion

B. Phenomenological models

1. Chiral active Brownian motion

*giovanni.volpe@physics.gu.se
2. Models for active particle reorientation $\quad 7$

3. Nonspherical active particles $\quad 7$

4. Modeling active motion with external forces and torques

5. Numerical considerations

C. Effective diffusion coefficient and effective temperature

D. Biological microswimmers 10

E. Artificial microswimmers 10

1. Propulsion by local energy conversion 11 
2. Propulsion by external fields

3. Synthesis of Janus particles

III. Hydrodynamics

A. Microhydrodynamics of self-propulsion

B. Particle-particle hydrodynamic interactions

C. Hydrodynamic coupling to walls

D. Non-Newtonian media

IV. Interacting Particles

A. Classification of particle interactions

1. Aligning interactions, Vicsek model, and swarming

B. Collective behaviors of active particles

1. Clustering and living crystals

2. Self-jamming and active microrheology

3. Active turbulence

C. Mixtures of active and passive particles

1. Active doping

2. Phase separation and turbulent behavior

3. Active baths

4. Directed motion and gears

5. Active depletion

6. Flexible passive particles and polymers

V. Complex Environments

A. Interaction with a wall

B. Active particles in a confined geometry

1. Non-Boltzmann position distributions for active particles

2. Active matter forces and equation of state

3. Collective behaviors in confined geometries

C. Interaction with obstacles

1. Capture and concentration of active particles

2. Ratchet effects and directed motion

3. Motion rectification in a microchannel

4. Extended landscapes of obstacles

5. Subdiffusion and trapping of microswimmers

D. Sorting of microswimmers

1. Static patterns

2. Chiral particle separation

VI. Toward the Nanoscale

VII. Outlook and Future Directions

Acknowledgments

References

\section{INTRODUCTION}

Active matter systems are able to take energy from their environment and drive themselves far from equilibrium (Ramaswamy, 2010). Thanks to this property, they feature a series of novel behaviors that are not attainable by matter at thermal equilibrium, including, for example, swarming and the emergence of other collective properties (Schweitzer, 2007). Their study provides great hope to uncover new physics and, simultaneously, to lead to the development of novel strategies for designing smart devices and materials. In recent years, a significant and growing effort has been devoted to advancing this field and to explore its applications in a diverse set of disciplines such as statistical physics (Ramaswamy, 2010), biology (Viswanathan et al., 2011), robotics (Brambilla et al., 2013), social transport (Helbing, 2001), soft matter (Marchetti et al., 2013), and biomedicine (Wang and Gao, 2012).
An important example of active matter is constituted by natural and artificial objects capable of self-propulsion. Selfpropelled particles were originally studied to model the swarm behavior of animals at the macroscale. Reynolds (1987) introduced a "Boids model" to simulate the aggregate motion of flocks of birds, herds of land animals, and schools of fish within computer graphics applications. Vicsek et al. (1995) then introduced his namesake model as a special case. In the Vicsek model, a swarm is modeled by a collection of selfpropelling particles that move with a constant speed but tend to align with the average direction of motion of the particles in their local neighborhood (Czirók and Vicsek, 2000; Chaté, Ginelli, Grégoire, Peruani, and Raynaud, 2008). Swarming systems give rise to emergent behaviors, which occur at many different scales; furthermore, some of these behaviors are turning out to be robust and universal, e.g., they are independent of the type of animals constituting the swarm (Buhl et al., 2006). It has in fact become a challenge for theoretical physics to find minimal statistical models that capture these features (Toner, Tu, and Ramaswamy, 2005; Li, Lukeman, and Edelstein-Keshet, 2008; Bertin, Droz, and Grégoire, 2009).

Self-propelled Brownian particles, in particular, have come under the spotlight of the physical and biophysical research communities. These active particles are biological or manmade microscopic and nanoscopic objects that can propel themselves by taking up energy from their environment and converting it into directed motion (Ebbens and Howse, 2010). On the one hand, self-propulsion is a common feature in microorganisms (Lauga and Powers, 2009; Cates, 2012; Poon, 2013) and allows for a more efficient exploration of the environment when looking for nutrients or running away from toxic substances (Viswanathan et al., 2011). A paradigmatic example is the swimming behavior of bacteria such as Escherichia coli (Berg, 2004). On the other hand, tremendous progress has recently been made toward the fabrication of artificial microswimmers and nanoswimmers that can selfpropel based on different propulsion mechanisms. Some characteristic examples of artificial self-propelled Brownian particles are provided in Fig. 1 and Table I.

While the motion of passive Brownian particles is driven by equilibrium thermal fluctuations due to random collisions with the surrounding fluid molecules (Babič, Schmitt, and Bechinger, 2005), self-propelled Brownian particles exhibit an interplay between random fluctuations and active swimming that drives them into a far-from-equilibrium state (Erdmann et al., 2000; Schweitzer, 2007; Hänggi and Marchesoni, 2009; Hauser and Schimansky-Geier, 2015). Thus, their behavior can be explained and understood only within the framework of nonequilibrium physics (Cates, 2012) for which they provide ideal model systems.

From a more applied perspective, active particles provide great hope to address some challenges that our society is currently facing - in particular, personalized health care, environmental sustainability, and security (Nelson, Kaliakatsos, and Abbott, 2010; Wang and Gao, 2012; Patra et al., 2013; Abdelmohsen et al., 2014; Gao and Wang, 2014; Ebbens, 2016). These potential applications can be built around the core functionalities of self-propelled Brownian particles, i.e., transport, sensing, and manipulation. In fact, 


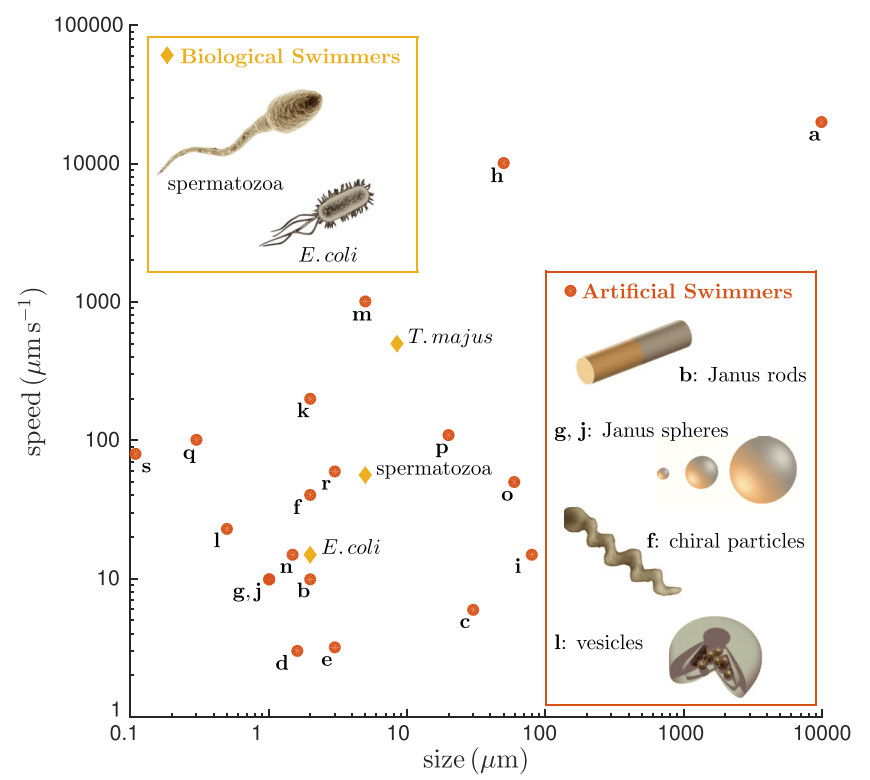

FIG. 1. Self-propelled Brownian particles are biological or manmade objects capable of taking up energy from their environment and converting it into directed motion. They are microscopic and nanoscopic in size and have propulsion speeds (typically) up to a fraction of a millimeter per second. The letters correspond to the artificial microswimmers in Table I. The insets show examples of biological and artificial swimmers. For the artificial swimmers four main recurrent geometries can be identified so far: Janus rods, Janus spheres, chiral particles, and vesicles.

these micromachines and nanomachines hold the promise of performing key tasks in an autonomous, targeted, and selective way. The possibility of designing, using, and controlling microswimmers and nanoswimmers in realistic settings of operation is tantalizing as a way to localize, pick up, and deliver nanoscopic cargoes in several applications - from the targeted delivery of drugs, biomarkers, or contrast agents in health care applications (Nelson, Kaliakatsos, and Abbott, 2010; Wang and Gao, 2012; Patra et al., 2013; Abdelmohsen et al., 2014) to the autonomous depollution of water and soils contaminated because of bad waste management, climate changes, or chemical terroristic attacks in sustainability and security applications (Gao and Wang, 2014).

The field of active matter is now confronted with various open challenges that will keep researchers busy for decades to come. First, there is a need to understand how living and inanimate active matter systems develop social and (possibly) tunable collective behaviors that are not attainable by their counterparts at thermal equilibrium. Then, there is a need to understand the dynamics of active particles in real-life environments (e.g., in living tissues and porous soils), where randomness, patchiness, and crowding can either limit or enhance how biological and artificial microswimmers perform a given task, such as finding nutrients or delivering a nanoscopic cargo. Finally, there is still a strong need to effectively scale down to the nanoscale our current understanding of active matter systems.

With this review, we provide a guided tour through the basic principles of self-propulsion at the microscale and nanoscale, the development of artificial self-propelling microparticles and nanoparticles, and their application to the study of farfrom-equilibrium phenomena, as well as through the open challenges that the field is now facing.

\section{NONINTERACTING ACTIVE PARTICLES IN HOMOGENOUS ENVIRONMENTS}

Before proceeding to analyze the behavior of active particles in crowded and complex environments, we set the stage by considering the simpler (and more fundamental) case of individual active particles in homogeneous environments, i.e., without obstacles or other particles. We first introduce a simple model of an active Brownian particle, ${ }^{1}$ which will permit us to understand the main differences between passive and active Brownian motion (Sec. II.A) and serve as a starting point to discuss the basic mathematical models for active motion (Sec. II.B). We then introduce the concepts of effective diffusion coefficient and effective temperature for selfpropelled Brownian particles, as well as their limitations, i.e., differences between systems at equilibrium at a higher temperature and systems out of equilibrium (Sec. II.C). We then briefly review biological microswimmers (Sec. II.D). Finally, we conclude with an overview of experimental achievements connected to the realization of artificial microswimmers and nanoswimmers including a discussion of the principal experimental approaches that have been proposed so far to build active particles (Sec. II.E).

\section{A. Brownian motion versus active Brownian motion}

In order to start acquiring some basic understanding of the differences between passive and active Brownian motion, a good (and pedagogic) approach is to compare twodimensional trajectories of single spherical passive and active particles of equal (hydrodynamic) radius $R$ in a homogenous environment, i.e., where no physical barriers or other particles are present and where there is a homogeneous and constant distribution of the energy source for the active particle.

The motion of a passive Brownian particle is purely diffusive with translational diffusion coefficient

$$
D_{\mathrm{T}}=\frac{k_{\mathrm{B}} T}{6 \pi \eta R},
$$

where $k_{\mathrm{B}}$ is the Boltzmann constant, $T$ is the absolute temperature, and $\eta$ is the fluid viscosity. The particle also undergoes rotational diffusion with a characteristic time scale $\tau_{\mathrm{R}}$ given by the inverse of the particle's rotational diffusion coefficient

\footnotetext{
${ }^{1}$ The term "active Brownian particle" has mainly been used in the literature to denote the specific, simplified model of active matter described in this section, which consists of repulsive spherical particles that are driven by a constant force whose direction rotates by thermal diffusion. Here we use the term active Brownian particle when we refer to this specific model and its straightforward generalizations (see Sec. II.B.1), while we use the terms "active particle" or "self-propelled particle" when we refer to more general systems.
} 
TABLE I. Examples of experimentally realized artificial microswimmers and relative propulsion mechanisms. The letters in the first column correspond to the examples plotted in Fig. 1.

\begin{tabular}{|c|c|c|c|c|c|}
\hline & Microswimmer & Propulsion mechanism & Medium & Dimensions & $\begin{array}{l}\text { Maximum } \\
\text { speed }\end{array}$ \\
\hline $\mathrm{a}$ & $\begin{array}{l}\text { Polydimethylsiloxane platelets } \\
\text { coated with Pt (Ismagilov } \\
\text { et al., 2002) }\end{array}$ & $\begin{array}{l}\text { Bubbles generated in a } \mathrm{H}_{2} \mathrm{O}_{2} \\
\text { aqueous solution by } \\
\text { asymmetric patterns of } \mathrm{Pt}\end{array}$ & $\mathrm{H}_{2} \mathrm{O}_{2}$ aqueous meniscus & $1 \mathrm{~cm}$ & $2 \mathrm{~cm} \mathrm{~s}^{-1}$ \\
\hline $\mathrm{b}$ & $\begin{array}{l}\text { Rod-shaped particles consisting } \\
\text { of } \mathrm{Au} \text { and Pt segments (Paxton } \\
\text { et al., 2004) }\end{array}$ & $\begin{array}{l}\text { Catalysis of oxygen at the Pt end } \\
\text { of the rod }\end{array}$ & $\begin{array}{l}\text { Near a boundary in } \mathrm{H}_{2} \mathrm{O}_{2} \\
\text { acqueous solution }\end{array}$ & $\begin{array}{l}2 \mu \mathrm{m} \text { (length), } \\
370 \mathrm{~nm} \text { (width) }\end{array}$ & $10 \mu \mathrm{m} \mathrm{s}^{-1}$ \\
\hline $\mathrm{c}$ & $\begin{array}{l}\text { Linear chains of DNA-linked } \\
\text { magnetic colloidal particles } \\
\text { attached to red blood cells } \\
\text { (Dreyfus et al., 2005) }\end{array}$ & $\begin{array}{l}\text { External actuation of the flexible } \\
\text { artificial flagella by oscillating } \\
\text { magnetic fields }\end{array}$ & Aqueous solution & $30 \mu \mathrm{m}$ & $6 \mu \mathrm{m} \mathrm{s}^{-1}$ \\
\hline d & $\begin{array}{l}\text { Janus spherical particles with a } \\
\text { catalytic Pt patch (Howse } \\
\text { et al., 2007) }\end{array}$ & $\begin{array}{l}\text { Self-diffusiophoresis catalyzed } \\
\text { by a chemical reaction on the } \\
\text { Pt surface }\end{array}$ & $\mathrm{H}_{2} \mathrm{O}_{2}$ aqueous solution & $1.6 \mu \mathrm{m}$ & $3 \mu \mathrm{m} \mathrm{s}^{-1}$ \\
\hline $\mathrm{e}$ & $\begin{array}{l}\text { DNA-linked anisotropic doublets } \\
\text { composed of paramagnetic } \\
\text { colloidal particles (Tierno } \\
\text { et al., 2008) }\end{array}$ & $\begin{array}{l}\text { Rotation induced by a rotating } \\
\text { magnetic field }\end{array}$ & $\begin{array}{l}\text { Near a boundary in } \\
\text { aqueous solution }\end{array}$ & $3 \mu \mathrm{m}$ & $3.2 \mu \mathrm{m} \mathrm{s}^{-1}$ \\
\hline f & $\begin{array}{l}\text { Chiral colloidal propellers } \\
\text { (Ghosh and Fischer, 2009) }\end{array}$ & $\begin{array}{l}\text { External actuation by a magnetic } \\
\text { field }\end{array}$ & Aqueous solution & $\begin{array}{l}2 \mu \mathrm{m} \text { (length), } \\
250 \mathrm{~nm} \text { (width) }\end{array}$ & $40 \mu \mathrm{m} \mathrm{s}^{-1}$ \\
\hline g & $\begin{array}{l}\text { Janus particles half-coated with } \\
\text { Au (Jiang, Yoshinaga, and } \\
\text { Sano, 2010) }\end{array}$ & $\begin{array}{l}\text { Self-thermophoresis due to local } \\
\text { heating at the Au cap }\end{array}$ & Aqueous solution & $1 \mu \mathrm{m}$ & $10 \mu \mathrm{m} \mathrm{s}^{-1}$ \\
\hline $\mathrm{h}$ & $\begin{array}{l}\text { Catalytic microjets (Sanchez } \\
\quad \text { et al., 2011) }\end{array}$ & $\begin{array}{l}\mathrm{H}_{2} \mathrm{O}_{2} \text { catalysis on the internal } \\
\text { surface of the microjet }\end{array}$ & $\mathrm{H}_{2} \mathrm{O}_{2}$ aqueous solution & $\begin{array}{l}50 \mu \mathrm{m} \text { (length), } \\
1 \mu \mathrm{m} \text { (width) }\end{array}$ & $10 \mathrm{~mm} \mathrm{~s}^{-1}$ \\
\hline $\mathrm{i}$ & $\begin{array}{l}\text { Water droplets containing } \\
\text { bromine (Thutupalli, Seemann, } \\
\text { and Herminghaus, 2011) }\end{array}$ & $\begin{array}{l}\text { Marangoni flow induced by a } \\
\text { self-sustained bromination } \\
\text { gradient along the drop surface }\end{array}$ & $\begin{array}{l}\text { Oil phase containing a } \\
\text { surfactant }\end{array}$ & $80 \mu \mathrm{m}$ & $15 \mu \mathrm{m} \mathrm{s}^{-1}$ \\
\hline $\mathrm{j}$ & $\begin{array}{l}\text { Janus particles with light- } \\
\text { absorbing patches (Volpe et al., } \\
\text { 2011; Buttinoni et al., 2012; } \\
\text { Kümmel et al., 2013) }\end{array}$ & $\begin{array}{l}\text { Local demixing of a critical } \\
\text { mixture due to heating } \\
\text { associated with localized } \\
\text { absorption of light }\end{array}$ & $\begin{array}{l}\text { Critical mixture (e.g., } \\
\text { water-2,6-lutidine) }\end{array}$ & $0.1-10 \mu \mathrm{m}$ & $10 \mu \mathrm{m} \mathrm{s}^{-1}$ \\
\hline $\mathrm{k}$ & $\begin{array}{l}\text { Rod-shaped particles consisting } \\
\text { of } \mathrm{Au} \text { and } \mathrm{Pt}(\text { or } \mathrm{Au} \text { and } \mathrm{Ru} \text { ) } \\
\text { segments (Wang et al., 2012) }\end{array}$ & $\begin{array}{l}\text { Self-acoustophoresis in a } \\
\text { ultrasonic standing wave }\end{array}$ & Aqueous solution & $\begin{array}{l}1-3 \mu \mathrm{m} \text { (length), } \\
300 \mathrm{~nm} \text { (width) }\end{array}$ & $200 \mu \mathrm{m} \mathrm{s}^{-1}$ \\
\hline 1 & $\begin{array}{l}\text { Pt-loaded stomatocytes (Wilson, } \\
\text { Nolte, and van Hest, 2012) }\end{array}$ & $\begin{array}{l}\text { Bubbles generated in a } \mathrm{H}_{2} \mathrm{O}_{2} \\
\text { aqueous solution by entrapped } \\
\text { Pt nanoparticles }\end{array}$ & $\mathrm{H}_{2} \mathrm{O}_{2}$ acqueous solution & $0.5 \mu \mathrm{m}$ & $23 \mu \mathrm{m} \mathrm{s}^{-1}$ \\
\hline $\mathrm{m}$ & $\begin{array}{l}\text { Colloidal rollers made of PMMA } \\
\text { beads (Bricard et al., 2013) }\end{array}$ & $\begin{array}{l}\text { Spontaneous charge symmetry } \\
\text { breaking resulting in a net } \\
\text { electrostatic torque }\end{array}$ & $\begin{array}{l}\text { Conducting fluid } \\
\text { (hexadecane solution) }\end{array}$ & $5 \mu \mathrm{m}$ & $1 \mathrm{~mm} \mathrm{~s}^{-1}$ \\
\hline $\mathrm{n}$ & $\begin{array}{l}\text { Polymeric spheres encapsulating } \\
\text { most of an antiferromagnetic } \\
\text { hematite cube (Palacci et al., } \\
\text { 2013) }\end{array}$ & $\begin{array}{l}\text { Self-phoretic motion near a } \\
\text { boundary due to the } \\
\text { decomposition of } \mathrm{H}_{2} \mathrm{O}_{2} \text { by the } \\
\text { hematite cube when } \\
\text { illuminated by ultraviolet light }\end{array}$ & $\begin{array}{l}\text { Near a boundary in } \mathrm{H}_{2} \mathrm{O}_{2} \\
\text { acqueous solution }\end{array}$ & $1.5 \mu \mathrm{m}$ & $15 \mu \mathrm{m} \mathrm{s}^{-1}$ \\
\hline $\mathrm{o}$ & Water droplets (Izri et al., 2014) & $\begin{array}{l}\text { Water solubilization by the } \\
\text { reverse micellar solution }\end{array}$ & $\begin{array}{l}\text { Oil phase with surfactants } \\
\text { above the critical } \\
\text { micellar solution }\end{array}$ & $60 \mu \mathrm{m}$ & $50 \mu \mathrm{m} \mathrm{s}^{-1}$ \\
\hline $\mathrm{p}$ & $\begin{array}{l}\text { Janus microspheres with } \mathrm{Mg} \\
\text { core, Au nanoparticles, and } \\
\mathrm{TiO}_{2} \text { shell layer (J. Li et al., } \\
\text { 2014) }\end{array}$ & $\begin{array}{l}\text { Bubble thrust generated from the } \\
\text { Mg-water reaction }\end{array}$ & Aqueous solution & $20 \mu \mathrm{m}$ & $110 \mu \mathrm{m} \mathrm{s}^{-1}$ \\
\hline q & $\begin{array}{l}\text { Hollow mesoporous silica Janus } \\
\text { particles (Ma, Hahn, and } \\
\text { Sanchez, 2015; Ma et al., } \\
\text { 2015) }\end{array}$ & $\begin{array}{l}\text { Catalysis powered by Pt or by } \\
\text { three different enzymes } \\
\text { (catalase, urease, and glucose } \\
\text { oxidase) }\end{array}$ & Aqueous solution & $50-500 \mathrm{~nm}$ & $100 \mu \mathrm{m} \mathrm{s}^{-1}$ \\
\hline $\mathrm{r}$ & $\begin{array}{l}\text { Janus particles half coated with } \\
\text { Cr (Nishiguchi and Sano, } \\
\text { 2015) }\end{array}$ & ac electric field & Aqueous solution & $3 \mu \mathrm{m}$ & $60 \mu \mathrm{m} \mathrm{s}^{-1}$ \\
\hline S & $\begin{array}{l}\text { Enzyme-loaded polymeric } \\
\quad \text { vesicles (Joseph et al., 2016) }\end{array}$ & $\begin{array}{l}\text { Glucose catalysis powered by } \\
\text { catalase and glucose oxidase }\end{array}$ & Aqueous solution & $0.1 \mu \mathrm{m}$ & $80 \mu \mathrm{m} \mathrm{s}^{-1}$ \\
\hline
\end{tabular}




$$
D_{\mathrm{R}}=\tau_{\mathrm{R}}^{-1}=\frac{k_{\mathrm{B}} T}{8 \pi \eta R^{3}}
$$

From the above formulas, it is clear that while the translational diffusion of a particle scales with its linear dimension, its rotational diffusion scales with its volume. For example, for a particle with $R \approx 1 \mu \mathrm{m}$ in water, $D_{\mathrm{T}} \approx 0.2 \mu \mathrm{m}^{2} \mathrm{~s}^{-1}$ and $D_{\mathrm{R}} \approx$ $0.17 \operatorname{rad}^{2} \mathrm{~s}^{-1}\left(\tau_{\mathrm{R}} \approx 6 \mathrm{~s}\right)$, while for a particle 10 times smaller $(R \approx 100 \mathrm{~nm}), D_{\mathrm{T}} \approx 2 \mu \mathrm{m}^{2} \mathrm{~s}^{-1}$ is 1 order of magnitude larger but $D_{\mathrm{R}} \approx 170 \mathrm{rad}^{2} \mathrm{~s}^{-1}$ is 3 orders of magnitude larger $\left(\tau_{\mathrm{R}} \approx 6 \mathrm{~ms}\right)$.

In a homogeneous environment, the translational and rotational motions are independent from each other. Therefore, the stochastic equations of motion for a passive Brownian particle in a two-dimensional space are

$$
\dot{x}=\sqrt{2 D_{\mathrm{T}}} \xi_{x}, \quad \dot{y}=\sqrt{2 D_{\mathrm{T}}} \xi_{y}, \quad \dot{\varphi}=\sqrt{2 D_{\mathrm{R}}} \xi_{\varphi},
$$

where $[x, y]$ is the particle position, $\varphi$ is its orientation [Fig. 2(a)], and $\xi_{x}, \xi_{y}$, and $\xi_{\varphi}$ represent independent white noise stochastic processes with zero mean and correlation $\delta(t)$. Interestingly, the equations for each degree of freedom (i.e., $x, y$, and $\varphi$ ) are decoupled. Inertial effects are neglected because microscopic particles are typically in a low-Reynoldsnumber regime (Purcell, 1977). Some examples of the corresponding trajectories are illustrated in Fig. 2(b).

For a self-propelled particle with velocity $v$ instead, the direction of motion is itself subject to rotational diffusion, which leads to a coupling between rotation and translation. The corresponding stochastic differential equations are

$$
\begin{aligned}
\dot{x} & =v \cos \varphi+\sqrt{2 D_{\mathrm{T}}} \xi_{x}, \quad \dot{y}=v \sin \varphi+\sqrt{2 D_{\mathrm{T}}} \xi_{y}, \\
\dot{\varphi} & =\sqrt{2 D_{\mathrm{R}}} \xi_{\varphi} .
\end{aligned}
$$

Some examples of trajectories for various $v$ are shown in Figs. 2(c)-2(e): as $v$ increases, we obtain active trajectories that are characterized by directed motion at short time scales; however, over long time scales the orientation and direction of motion of the particle are randomized by its rotational diffusion (Howse et al., 2007).

To emphasize the difference between Brownian motion and active Brownian motion, it is instructive to consider the average particle trajectory given the initial position and orientation fixed at time $t=0$, i.e., $x(0)=y(0)=0$ and $\varphi(0)=0$. In the case of passive Brownian motion, this

(a)

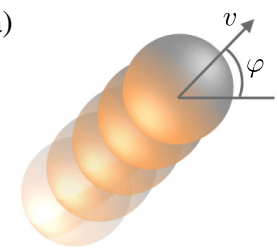

(b)

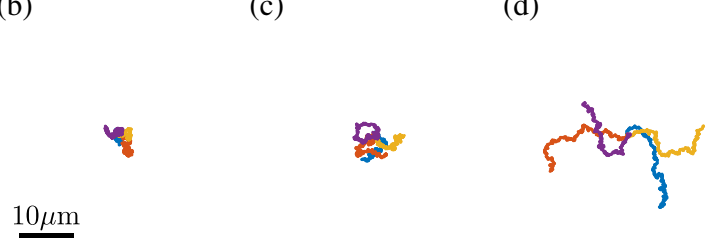

average vanishes by symmetry, i.e., $\langle x(t)\rangle=\langle y(t)\rangle \equiv 0$, where $\langle\cdots\rangle$ represents the ensemble average. For an active particle instead, the average is a straight line along the $x$ direction (determined by the prescribed initial orientation),

$\langle x(t)\rangle=\frac{v}{D_{\mathrm{R}}}\left[1-\exp \left(-D_{\mathrm{R}} t\right)\right]=v \tau_{\mathrm{R}}\left[1-\exp \left(-\frac{t}{\tau_{\mathrm{R}}}\right)\right]$,

while $\langle y(t)\rangle \equiv 0$ because of symmetry. This implies that, on average, an active Brownian particle will move along the direction of its initial orientation for a finite persistence length

$$
L=\frac{v}{D_{\mathrm{R}}}=v \tau_{\mathrm{R}}
$$

before its direction is randomized.

The relative importance of directed motion versus diffusion for an active Brownian particle can be characterized by its Péclet number

$$
\operatorname{Pe} \propto \frac{v}{\sqrt{D_{\mathrm{T}} D_{\mathrm{R}}}}
$$

where the proportionality sign is used because the literature is inconsistent about the value of the numerical prefactor. If $\mathrm{Pe}$ is small, diffusion is important, while if Pe is large, directed motion prevails.

The model for active Brownian motion described by Eqs. (4) can be straightforwardly generalized to the case of an active particle moving in three dimensions. In this case, the particle position is described by three Cartesian coordinates, i.e., $[x, y, z]$, and its orientation by the polar and azimuthal angles, i.e., $[\vartheta, \varphi]$, which perform a Brownian motion on the unit sphere (Carlsson, Ekholm, and Elvingson, 2010).

\section{B. Phenomenological models}

In this section, we extend the simple model introduced in Sec. II.A to describe the motion of more complex (and realistic) active Brownian particles. First, we introduce models that account for chiral active Brownian motion (Sec. II.B.1). We then consider more general models of active Brownian motion where reorientation occurs due to mechanisms other than rotational diffusion (Sec. II.B.2) and where the active particles are nonspherical (Sec. II.B.3). Finally, we discuss the use of external forces and torques when modeling active

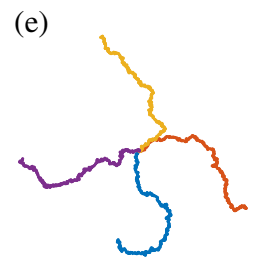

FIG. 2. Active Brownian particles in two dimensions. (a) An active Brownian particle in water $(R=1 \mu \mathrm{m}, \eta=0.001 \mathrm{Pas})$ placed at position $[x, y]$ is characterized by an orientation $\varphi$ along which it propels itself with speed $v$ while undergoing Brownian motion in both position and orientation. The resulting trajectories are shown for different velocities (b) $v=0 \mu \mathrm{m} \mathrm{s}^{-1}$ (Brownian particle), (c) $v=1 \mu \mathrm{m} \mathrm{s}^{-1}$, (d) $v=2 \mu \mathrm{m} \mathrm{s}^{-1}$, and (e) $v=3 \mu \mathrm{m} \mathrm{s}^{-1}$. With increasing values of $v$, the active particles move over longer distances before their direction of motion is randomized; four different 10-s trajectories are shown for each value of velocity. 
Brownian motion (Sec. II.B.4) and we provide some considerations about numerics (Sec. II.B.5).

Before proceeding further, we remark that in this section the microscopic swimming mechanism is completely ignored; in particular, hydrodynamic interactions are disregarded and only the observable effects of net motion are considered. While the models introduced here are phenomenological, they are very effective in describing the motion of microswimmers in homogenous environments. We cast this point in terms of the difference between "microswimmers" and active particles. Microswimmers are force-free and torque-free objects capable of self-propulsion in a (typically) viscous environment and, importantly, exhibit an explicit hydrodynamic coupling with the embedding solvent via flow fields generated by the swimming strokes they perform. Instead, active particles represent a much simpler concept consisting of self-propelled particles in an inert solvent, which provides only hydrodynamic friction and a stochastic momentum transfer. While the observable behavior of the two is the same in a homogenous environment and in the absence of interactions between particles, hydrodynamic interactions may play a major role in the presence of obstacles or other microswimmers. The simpler model of active particles delivers, however, good results in terms of the particle's behavior and is more intuitive. In fact, the self-propulsion of an active Brownian particle is implicitly modeled by using an effective force fixed in the particle's body frame. For this reason, in this review we typically consider active particles, while we discuss hydrodynamic interactions in Sec. III [see also Golestanian, Yeomans, and Uchida (2011), Marchetti et al. (2013), and Elgeti, Winkler, and Gompper (2015) for extensive reviews on the role of hydrodynamics in active matter systems]. We provide a more detailed theoretical justification of why active particles are a good model for microswimmers in Sec. II.B.4.

\section{Chiral active Brownian motion}

Swimming along a straight line, corresponding to the linearly directed Brownian motion considered until now, is the exception rather than the rule. In fact, ideal straight swimming occurs only if the left-right symmetry relative to the internal propulsion direction is not broken; even small deviations from this symmetry destabilize any straight motion and make it chiral. One can assign a chirality (or helicity) to the path, the sign of which determines whether the motion is clockwise (dextrogyre) or anticlockwise (levogyre). The result is a motion along circular trajectories in two dimensions (circle swimming) and along helical trajectories in three dimensions (helical swimming).

The occurrence of microorganisms swimming in circles was pointed out more than a century ago by Jennings (1901) and, since then, has been observed in many different situations, in particular, close to a substrate for bacteria (Berg and Turner, 1990; DiLuzio et al., 2005; Lauga et al., 2006; Hill et al., 2007; Shenoy et al., 2007; Schmidt et al., 2008) and spermatozoa (Woolley, 2003; Riedel, Kruse, and Howard, 2005; Friedrich and Jülicher, 2008). Likewise, helical swimming in three dimensions has been observed for various bacteria and sperm cells (Jennings, 1901; Brokaw, 1958, 1959; Crenshaw, 1996; Fenchel and Blackburn, 1999; Corkidi (a)

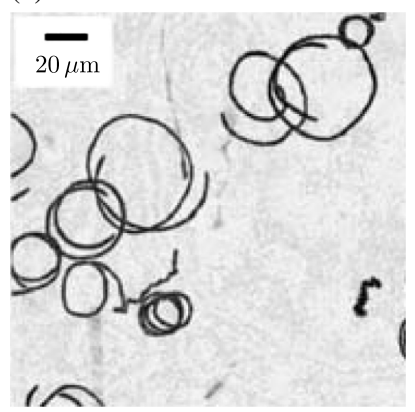

(c)

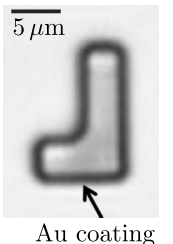

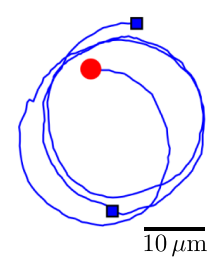

(b)

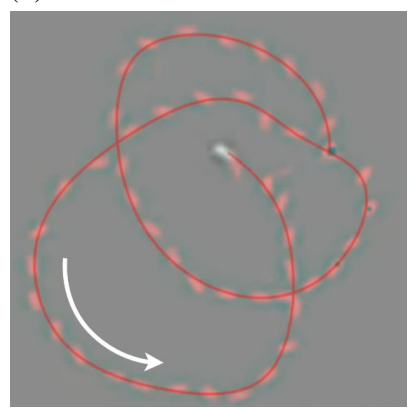

(d)

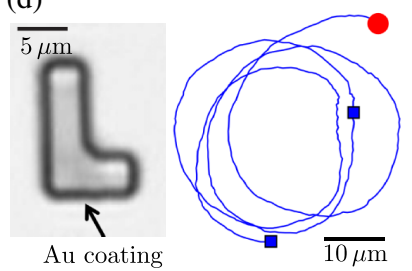

FIG. 3. Biological and artificial chiral active Brownian motion. (a) Phase-contrast video microscopy images showing E. coli cells swimming in circular trajectories near a glass surface. Superposition of 8-s video images. From Lauga et al., 2006. (b) Circular trajectories are also observed for E. coli bacteria swimming over liquid-air interfaces but the direction is reversed. From Di Leonardo et al., 2011. Trajectories of (c) dextrogyre and (d) levogyre artificial microswimmers driven by self-diffusiophoresis: in each plot, the red bullet corresponds to the initial particle position and the two blue squares to its position after 1 and 2 minutes. The insets show microscope images of two different swimmers with the Au coating (not visible in the bright-field image) indicated by an arrow. From Kümmel et al., 2013.

et al., 2008; Jékely et al., 2008). Figures 3(a) and 3(b) show examples of $E$. coli cells swimming in circular trajectories near a glass surface and at a liquid-air interface, respectively. Examples of nonliving but active particles moving in circles are spherical camphors at an air-water interface (Nakata et al., 1997) and chiral (L-shaped) colloidal swimmers on a substrate (Kümmel et al., 2013). Finally, trajectories of deformable active particles (Ohta and Ohkuma, 2009) and even of completely blinded and ear-plugged pedestrians (Obata et al., 2000) can possess significant circular characteristics.

The origin of chiral motion can be manifold. In particular, it can be due to an anisotropy in the particle shape, which leads to a translation-rotation coupling in the hydrodynamic sense (Kraft et al., 2013) or an anisotropy in the propulsion mechanism. Kümmel et al. (2013) experimentally studied an example where both mechanisms are simultaneously present [Figs. 3(c) and 3(d)]. Furthermore, even a cluster of nonchiral swimmers, which stick together by direct forces (Redner, Baskaran, and Hagan, 2013), by hydrodynamics, or just by the activity itself (Buttinoni et al., 2013; Palacci et al., 2013), will in general lead to situations of total nonvanishing torque on the cluster center (Kaiser, Popowa, and Löwen, 2015), thus leading to circling clusters (Schwarz-Linek et al., 2012). Finally, the particle rotation can be induced by external fields; a standard example is a magnetic field perpendicular to the plane of motion exerting a torque on the particles (Cēbers, 
2011). Even though the emergence of circular motion can be often attributed to hydrodynamic effects, in this section we focus on a phenomenological description and leave the proper hydrodynamic description to Sec. III.C.

For a two-dimensional chiral active Brownian particle [Fig. 4(a)], in addition to the random diffusion and the internal self-propulsion modeled by Eqs. (4), the particle orientation $\varphi$ also rotates with angular velocity $\omega$, where the sign of $\omega$ determines the chirality of the motion. The resulting set of equations describing this motion in two dimensions is (van Teeffelen and Löwen, 2008; Mijalkov and Volpe, 2013; Volpe, Gigan, and Volpe, 2014)

$$
\begin{aligned}
& \dot{x}=v \cos \varphi+\sqrt{2 D_{\mathrm{T}}} \xi_{x}, \quad \dot{y}=v \sin \varphi+\sqrt{2 D_{\mathrm{T}}} \xi_{y}, \\
& \dot{\varphi}=\omega+\sqrt{2 D_{\mathrm{R}}} \xi_{\varphi} .
\end{aligned}
$$

Some examples of trajectories are shown in Figs. 4(b)-4(d) for particles of decreasing radius. As the particle size decreases, the trajectories become less deterministic because the rotational diffusion, responsible for the reorientation of the particle direction, scales according to $R^{-3}$ [Eq. (2)]. The model given by Eqs. (8) can be straightforwardly extended to the helicoidal motion of a three-dimensional chiral active particle following an approach along the lines of the discussion at the end of Sec. II.A.

It is interesting to consider how the noise-averaged trajectory given in Eq. (5) changes in the presence of chiral motion. In this case, the noise-averaged trajectory has the shape of a logarithmic spiral, i.e., a spira mirabilis (van Teeffelen and Löwen, 2008), which in polar coordinates is written as

$$
\rho \propto \exp \left\{-D_{\mathrm{R}}[\varphi-\varphi(0)] / \omega\right\}
$$

where $\rho$ is the radial coordinate and $\varphi$ is the azimuthal coordinate. In three dimensions, the noise-averaged trajectory is a concho spiral (Wittkowski and Löwen, 2012), which is the generalization of the logarithmic spiral. Stochastic helical swimming was recently investigated in Colonial Choanoflagellates (Kirkegaard, Marron, and Goldstein, 2016).

\section{Models for active particle reorientation}

The simple models presented so far, and, in particular, the one discussed in Sec. II.A, consider an active particle whose velocity is constant in modulus and whose orientation

(a)

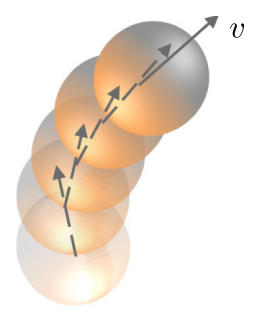

(b)

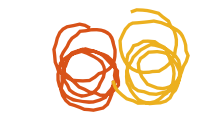

$5 \mu \mathrm{m}$

undergoes free diffusion. This type of dynamics, which we refer to as rotational diffusion dynamics [Fig. 5(a)], is often encountered in the case of self-propelling Janus colloids (Howse et al., 2007; Buttinoni et al., 2012; Palacci et al., 2013). There are, however, other processes that generate active Brownian motion; here we consider, in particular, the run-andtumble dynamics and the Gaussian noise dynamics (Koumakis, Maggi, and Di Leonardo, 2014). More general models include velocity- and space-dependent friction (Taktikos, Zaburdaev, and Stark, 2011; Romanczuk et al., 2012; Babel, ten Hagen, and Löwen, 2014). It has also been recently speculated that finite-time correlations in the orientational dynamics can affect the swimmer's diffusivity (Ghosh et al., 2015).

The run-and-tumble dynamics [Fig. 5(b)] were introduced to describe the motion of E. coli bacteria (Berg and Turner, 1979; Schnitzer, Block, and Berg, 1990; Berg, 2004). They consist of a random walk that alternates linear straight runs at constant speed with Poisson-distributed reorientation events called tumbles. Even though their microscopic (short-time) dynamics are different, their long-time diffusion properties are equivalent to those of the rotational diffusion dynamics described in Sec. II.A (Tailleur and Cates, 2008; Cates and Tailleur, 2013; Solon, Cates, and Tailleur, 2015).

In the Gaussian noise dynamics [Fig. 5(c)], the active particle velocity (along each direction) fluctuates as an Ornstein-Uhlenbeck process (Uhlenbeck and Ornstein, 1930). This is, for example, a good model for the motion of colloidal particles in a bacterial bath, where multiple interactions with the motile bacteria tend to gradually change the direction and amplitude of the particle's velocity, at least as long as the concentration is not so high to give rise to collective phenomena (Wu and Libchaber, 2000).

Finally, we also consider the interesting limit of the rotational diffusion dynamics when the rotational diffusion is zero, or similarly in the run-and-tumble dynamics when the run time is infinite. In this case, the equations of motion of the active particle contain no stochastic terms and the particle keeps on moving ballistically along straight lines until it interacts with some obstacles or other particles. Such a limit is reached, e.g., for sufficiently large active colloids or for active colloids moving through an extremely viscous fluid.

\section{Nonspherical active particles}

The models presented until now, in particular, Eqs. (4) and (8), are valid for spherical active particles. However, while

(c)

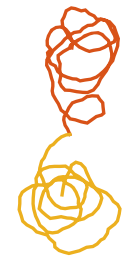

(d)

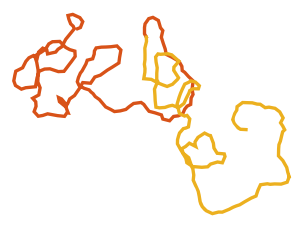

FIG. 4. Chiral active Brownian motion in two dimensions. (a) A two-dimensional chiral active Brownian particle has a deterministic angular velocity $\omega$ that, if the particle's speed $v>0$, entails a rotation around an effective external axis. (b)-(d) Sample trajectories of dextrogyre (red, dark gray) and levogyre (yellow, light gray) active chiral particles with $v=30 \mu \mathrm{m} \mathrm{s}^{-1}, \omega=10 \mathrm{rad} \mathrm{s}^{-1}$, and different radii $[R=1000,500$, and $250 \mathrm{~nm}$ for (b), (c), and (d), respectively]. As the particle size decreases, the trajectories become less deterministic because the rotational diffusion, responsible for the reorientation of the particle direction, scales according to $R^{-3}$ [Eq. (2)]. 
(a)

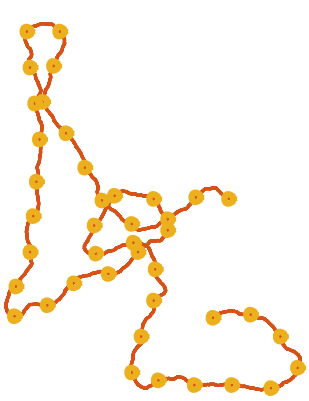

(b)

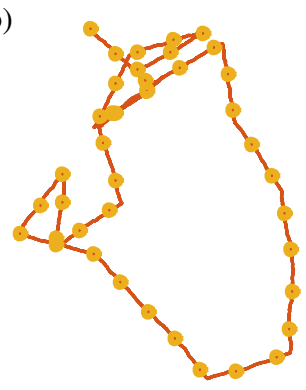

(c)

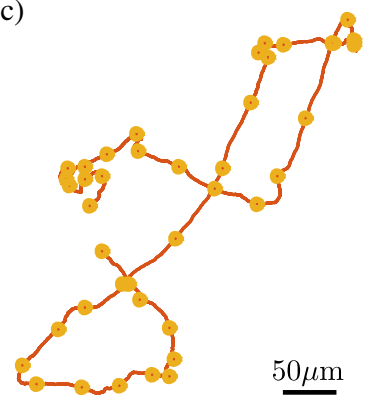

FIG. 5. Sample trajectories of active Brownian particles corresponding to different mechanisms generating active motion: (a) rotational diffusion dynamics, (b) run-and-tumble dynamics, and (c) Gaussian noise dynamics. The dots correspond to the particle position sampled every $5 \mathrm{~s}$.

most active particles considered in experiments and simulations are spherically or axially symmetric, many bacteria and motile microorganisms considerably deviate from such ideal shapes and this strongly alters their swimming properties.

In order to understand how we can derive the equations of motion for nonspherical active Brownian particles, it is useful to rewrite in a vectorial form the model presented in Sec. II.A for the simpler case of a spherical active particle:

$$
\gamma \dot{\mathbf{r}}=F \hat{\mathbf{e}}+\xi
$$

where $\gamma=6 \pi \eta R$ denotes the particle's Stokes friction coefficient (for a sphere of radius $R$ with sticky boundary conditions at the particle surface), $\mathbf{r}$ is its position vector, $F$ is an effective force acting on the particle, $\hat{\mathbf{e}}$ is its orientation unit vector, and $\boldsymbol{\xi}$ is a random vector with zero mean and correlation $2 k_{\mathrm{B}} T \gamma \rrbracket \delta(t)$, where $\rrbracket$ is the identity matrix in the appropriate number of dimensions. If the particle's orientation does not change, i.e., $\hat{\mathbf{e}}(t) \equiv \hat{\mathbf{e}}(0)$ (e.g., being fixed by an external aligning magnetic field), the particle swims with a self-propulsion speed $v=F / \gamma$ along its orientation $\hat{\mathbf{e}}$ and its trajectory is trivially given by $\mathbf{r}(t)=\mathbf{r}(0)+v t \hat{\mathbf{e}}(0)$. If the particle orientation can instead change, e.g., if $\hat{\mathbf{e}}$ is subject to rotational diffusion, the particle will perform active Brownian motion.

We can now generalize these simple considerations for a spherical particle to more complex shapes as systematically discussed by ten Hagen et al. (2015). When the particle has a rigid anisotropic shape, the resulting equations of motion can be written in compact form as

$$
\mathfrak{H} \cdot \mathbf{V}=\mathbf{K}+\chi,
$$

where $\mathbb{W}$ is the grand resistance matrix or hydrodynamic friction tensor (see also Sec. III.A) (Happel and Brenner, 1991; Fernandes and de la Torre, 2002), $\mathbf{V}=[\mathbf{v}, \boldsymbol{\omega}]$ is a generalized velocity with $\boldsymbol{v}$ and $\boldsymbol{\omega}$ the particle's translational and angular velocities, $\mathbf{K}=[\mathbf{F}, \mathbf{T}]$ is a generalized force with $\mathbf{F}$ and $\mathbf{T}$ the effective force and torque acting on the particle, and $\chi$ is a random vector with correlation $2 k_{\mathrm{B}} T \llbracket \delta(t)$. Equation (11) is best understood in the body frame of the moving particle where $\mathbb{H}, \mathbf{K}$, and $\mathbf{V}$ are constant, but it can also be transformed to the laboratory frame (Wittkowski and Löwen, 2012). In the deterministic limit (i.e., $\chi=\mathbf{0}$ ), the particle trajectories are straight lines if $\boldsymbol{\omega}=\mathbf{0}$, and circles in two dimensions (or helices in three dimensions) if $\boldsymbol{\omega} \neq \mathbf{0}$ (Friedrich and Jülicher, 2009; Wittkowski and Löwen, 2012). In the opposite limit when $\mathbf{K}=\mathbf{0}$, we recover the case of a free Brownian particle, which however features nontrivial dynamical correlations (Fernandes and de la Torre, 2002; Makino and Doi, 2004; Kraft et al., 2013; Cichocki, EkielJezewska, and Wajnryb, 2015).

\section{Modeling active motion with external forces and torques}

Equation (10) describes the motion of a spherical active particle using an effective "internal" force $F=\gamma v$ fixed in the particle's body frame. $F$ is identical to the force acting on a hypothetical spring whose ends are bound to the microswimmers and to the laboratory (Takatori, Yan, and Brady, 2014); hence, it can be directly measured, at least in principle. While this force can be viewed as a special force field $\mathbf{F}(\mathbf{r}, \hat{\mathbf{e}})=F \hat{\mathbf{e}}$ experienced by the particle, it is clearly nonconservative, i.e., it cannot be expressed as a spatial gradient of a scalar potential. The advantage in modeling self-propulsion by an effective driving force is that this force can be straightforwardly added to all other existing forces, e.g., body forces from real external fields (like gravity or confinement), forces stemming from the interaction with other particles, and random forces mimicking the random collisions with the solvent. This keeps the model simple, flexible, and transparent. This approach has been followed by many recent works; see, e.g., Chen and Leung (2006), Peruani, Deutsch, and Bär (2006), Li, Lukeman, and Edelstein-Keshet (2008), Mehandia and Prabhu (2008), Wensink and Löwen (2008), van Teeffelen and Löwen (2008), Angelani, Costanzo, and Di Leonardo (2011), ten Hagen, Wittkowski, and Löwen (2011), Bialké, Speck, and Löwen (2012), Kaiser, Wensink, and Löwen (2012), McCandlish, Baskaran, and Hagan (2012), Wensink and Löwen (2012), Wittkowski and Löwen (2012), Yang et al. (2012), Elgeti and Gompper (2013), Kaiser et al. (2013), Mijalkov and Volpe (2013), Redner, Hagan, and Baskaran (2013), Reichhardt and OlsonReichhardt (2013a), Costanzo et al. (2014), Fily, Henkes, and Marchetti (2014), and Wang et al. (2014).

These simple considerations for a spherical active particle can be generalized to more complex situations, such as to Eqs. (11) for nonspherical active particles. In general, the following considerations hold to decide whether a model 
based on effective forces and torques can be applied safely. On the one hand, the effective forces and torques can be used if we consider a single particle in an unbounded fluid whose propulsion speed is a generic explicit function of time (Babel, ten Hagen, and Löwen, 2014) or of the particle's position (Magiera and Brendel, 2015). On the other hand, body forces and torques arising, e.g., from an external field or from (nonhydrodynamic) particle interactions can simply be added to the effective forces and torques, under the sole assumption that the presence of the body forces and torques should not affect the self-propulsion mechanism itself. A classical counterexample to this assumption is bimetallic nanorods driven by electrophoresis in an external electric field (Paxton et al., 2004, 2006), as the external electric field perturbs the transport of ions through the rod and the screening around it and thus significantly affects its propagation (Brown and Poon, 2014).

In order to avoid potential confusion, we remark that the use of effective forces does not imply that the solvent flow field is modeled correctly; contrarily, the flow field is not considered at all. When the propagation is generated by a nonreciprocal mechanical motion of different parts of the swimmer, any internal motion should fulfill Newton's third law such that the total force acting on the swimmer is zero at any time. As we see in more detail in Sec. III, this implies that the solvent velocity field $\mathbf{u}(\mathbf{r})$ around a swimmer does not decay as a force monopole [i.e., $\mathbf{u}(\mathbf{r}) \propto 1 / r$, as if the particle were dragged by a constant external force field], but (much faster) as a force dipole [i.e., $\mathbf{u}(\mathbf{r}) \propto 1 / r^{2}$ ]. The notion of an effective internal force, therefore, seems to contradict this general statement that the motion of a swimmer is force free. The solution of this apparent contradiction is that the modeling via an effective internal force does not resolve the solvent velocity field but is just a coarse-grained effective description for swimming with a constant speed along the particle trajectory. Therefore, the concept of effective forces and torques is of limited utility when the solvent flow field, which is generated by the self-propelled particles, has to be taken into account explicitly. This applies, for example, to the far field of the solvent flow that governs the dynamics of a particle pair [and discriminates between pullers and pushers (Downton and Stark, 2009)], to the hydrodynamic interaction between a particle and an obstacle (Kreuter et al., 2013; Chilukuri, Collins, and Underhill, 2014; Takagi et al., 2014; Sipos et al., 2015), and to the complicated many-body hydrodynamic interactions in a dense suspension of swimmers (Kapral, 2008; Alexander, Pooley, and Yeomans, 2009; Gompper et al., 2009; Reigh, Winkler, and Gompper, 2012). Nonetheless, there are various situations where hydrodynamic interactions do not play a major role. This is the case for dry active matter (Marchetti et al., 2013), for effects close to a substrate where lubrication is dominating, and for highly crowded environments where the hydrodynamic interactions can cancel if no global flow is built up (Wioland et al., 2013).

\section{Numerical considerations}

Numerically, the continuous-time solution to the set of stochastic differential equations given by Eqs. (4), as well as for the other equations presented in this section, can be obtained by approximating it with a set of finite difference equations (Ermak and McCammon, 1978; Volpe and Volpe, 2013; Volpe, Gigan, and Volpe, 2014). Even though in most practical applications the simple first-order scheme works best, care has to be taken to choose the time step small enough; higher-order algorithms can also be employed to obtain faster convergence of the solution (Honerkamp, 1993; Kloeden and Pearson, 1999) and to deal correctly with interactions with obstacles or other particles (Behringer and Eichhorn, 2011; Behringer and Eichhorn, 2012).

\section{Effective diffusion coefficient and effective temperature}

As seen in Sec. II.A, when the speed $v$ of an active particle increases in a homogeneous environment (no crowding and no physical barriers), its trajectories (Fig. 2) are typically dominated by directed motion on short time scales and by an enhanced random diffusion at long times, the latter due to random changes in the swimming direction (Howse et al., 2007). These qualitative considerations can be made more precise by calculating the mean square displacement $\operatorname{MSD}(\tau)$ of the motion for both passive and active particles at different values of $v$, as shown in Fig. 6. The $\operatorname{MSD}(\tau)$ quantifies how a particle moves away from its initial position and can be calculated directly from a trajectory. For a passive Brownian particle, the $\operatorname{MSD}(\tau)$ in two dimensions is

$$
\operatorname{MSD}(\tau)=4 D_{\mathrm{T}} \tau
$$

which is valid for times significantly longer than the momentum relaxation time $\tau_{\mathrm{m}}=m / \gamma$ of the particle, where $m$ is the mass of the particle. ${ }^{2}$

For an active particle instead, the theoretical $\operatorname{MSD}(\tau)$ is given by (Franke and Gruler, 1990; Howse et al., 2007; Martens et al., 2012) ${ }^{3}$

$$
\operatorname{MSD}(\tau)=\left[4 D_{\mathrm{T}}+2 v^{2} \tau_{\mathrm{R}}\right] \tau+2 v^{2} \tau_{\mathrm{R}}^{2}\left[e^{-\tau / \tau_{\mathrm{R}}}-1\right]
$$

${ }^{2}$ To be more precise, the theoretical $\operatorname{MSD}(\tau)$ for a passive Brownian particle is given by the Ornstein-Uhlenbeck formula (Uhlenbeck and Ornstein, 1930), which in two dimensions reads

$$
\operatorname{MSD}(\tau)=4 D_{\mathrm{T}} \tau+\frac{4 k_{\mathrm{B}} T}{m} \tau_{\mathrm{m}}^{2}\left[e^{-\tau / \tau_{\mathrm{m}}}-1\right]
$$

At long time scales, the $\operatorname{MSD}(\tau)$ of a passive Brownian particle is therefore linear in time with a slope controlled by the particle's diffusion coefficient $D_{\mathrm{T}}$. This occurs for $\tau \gg \tau_{\mathrm{m}}$, where $\tau_{\mathrm{m}}$ for small colloidal particles is of the order of microseconds. In a liquid environment, furthermore, also the hydrodynamic memory of the fluid, i.e., the mass of the fluid displaced together with the particle, must be taken into account and can, in fact, significantly increase the effective momentum relaxation time (Lukić et al., 2005; Franosch et al., 2011; Pesce et al., 2014).

${ }^{3}$ Note that Eq. (13) is formally equal to the Ornstein-Uhlenbeck formula for the MSD of a Brownian particle with inertia, which describes the transition from the ballistic regime to the diffusive regime, although at a much shorter time scale than for active particles (Uhlenbeck and Ornstein, 1930). 


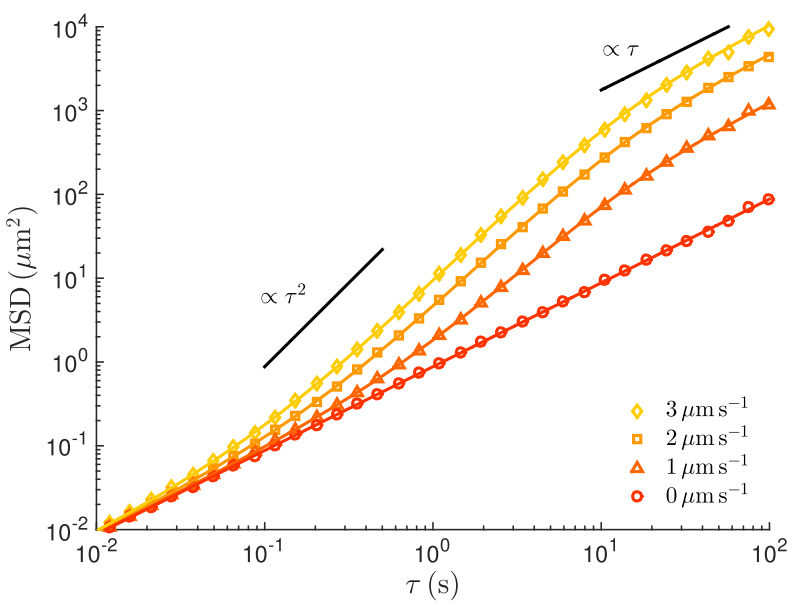

FIG. 6. Mean square displacement (MSD) of active Brownian particles and effective diffusion coefficients. Numerically calculated (symbols) and theoretical (lines) MSD for an active Brownian particle with velocity $v=0 \mu \mathrm{m} \mathrm{s}^{-1}$ (circles), $v=$ $1 \mu \mathrm{m} \mathrm{s}^{-1}$ (triangles), $v=2 \mu \mathrm{m} \mathrm{s}^{-1}$ (squares), and $v=3 \mu \mathrm{m} \mathrm{s}^{-1}$ (diamonds). For a passive Brownian particle $\left(v=0 \mu \mathrm{m} \mathrm{s}^{-1}\right.$, circles) the motion is always diffusive $[\operatorname{MSD}(\tau) \propto \tau]$, while for an active Brownian particle the motion is diffusive with diffusion coefficient $D_{\mathrm{T}}$ at very short time scales $[\operatorname{MSD}(\tau) \propto \tau$ for $\left.\tau \ll \tau_{\mathrm{R}}\right]$, ballistic at intermediate time scales $\left[\operatorname{MSD}(\tau) \propto \tau^{2}\right.$ for $\tau \approx \tau_{\mathrm{R}}$, and again diffusive but with an enhanced diffusion coefficient at long time scales $\left[\operatorname{MSD}(\tau) \propto \tau\right.$ for $\left.\tau \gg \tau_{\mathrm{R}}\right]$.

This expression for the MSD holds whenever the active speed component is characterized by an exponential decay. This is the case, in particular, for all models for active particle reorientation we considered in Sec. II.B.2, i.e., rotational diffusion, run-and-tumble dynamics, and Gaussian noise dynamics. At very short time scales, i.e., $\tau \ll \tau_{\mathrm{R}}$, this expression becomes $\operatorname{MSD}(\tau)=4 D_{\mathrm{T}} \tau$ and, thus, the motion is diffusive with the typical Brownian short-time diffusion coefficient $D_{\mathrm{T}}$. This diffusive short-time regime indeed can be seen in experiments if the strength of self-propulsion is not very large (Zheng et al., 2013). At slightly longer time scales, i.e., $\tau \approx \tau_{\mathrm{R}}, \operatorname{MSD}(\tau)=4 D_{\mathrm{T}} \tau+2 v^{2} \tau^{2}$ so that the motion is superdiffusive. At much longer time scales, i.e., $\tau \gg \tau_{\mathrm{R}}$, $\operatorname{MSD}(\tau)=\left[4 D_{\mathrm{T}}+2 v^{2} \tau_{\mathrm{R}}\right] \tau$ and, thus, the $\operatorname{MSD}(\tau)$ is proportional to $\tau$, since the rotational diffusion leads to a randomization of the direction of propulsion and the particle undergoes a random walk whose step length is the product of the propelling velocity $v$ and the rotational diffusion time $\tau_{\mathrm{R}}$ [equal to the persistence length given by Eq. (6)]. This leads to a substantial enhancement of the effective diffusion coefficient over the value $D_{\mathrm{T}}$, which corresponds to $D_{\text {eff }}=D_{\mathrm{T}}+\frac{1}{2} v^{2} \tau_{\mathrm{R}}$.

One might be tempted to think that the stationary states of active Brownian systems could resemble equilibrium states at a higher effective temperature

$$
T_{\mathrm{eff}}=\frac{\gamma D_{\mathrm{eff}}}{k_{\mathrm{B}}}=T+\frac{\gamma v^{2} \tau_{\mathrm{R}}}{2 k_{\mathrm{B}}} .
$$

This simple picture of active particles as hot colloids may be correct in some simple situations, such as dilute noninteracting active particles that sediment in a uniform external force field (Tailleur and Cates, 2009; Palacci et al., 2010; Maggi et al., 2013). However, as soon as interactions become important or external fields are inhomogeneous, one observes phenomena like clustering in repulsive systems or rectification effects (Koumakis, Maggi, and Di Leonardo, 2014; Volpe, Gigan, and Volpe, 2014) that are not compatible with the picture of a quasiequilibrium state at one effective temperature (Argun et al., 2016).

\section{Biological microswimmers}

Various kinds of biological microswimmers exist in nature, e.g., bacteria (Berg and Brown, 1972; Berg and Turner, 1990; Berg, 2004), unicellular protozoa (Machemer, 1972; Blake and Sleigh, 1974), and spermatozoa (Woolley, 2003; Riedel, Kruse, and Howard, 2005). Typically, the planktonic swimming motion of these microorganisms is generated by flagella or cilia powered by molecular motors (Lauga and Goldstein, 2012; Poon, 2013; Alizadehrad et al., 2015; Elgeti, Winkler, and Gompper, 2015). Alternative methods, such as crawling or swarming, do not involve swimming in a fluid but they rather require cells to move on a substrate or through a gel or porous material.

While many properties of the motion of biological microswimmers can be understood in terms of effective Langevin equations, seen in Sec. II.B, several models have been proposed to understand in more detail their microscopic mechanisms. Lighthill (1952) introduced a model for squirmers in a viscous fluid. The Lighthill model assumes that the movement of a spherical particle, covered by a deformable spherical envelope, is caused by an effective slip velocity between the particle and the solvent. This model was corrected to describe the metachronal wavelike beat of cilia densely placed on the surface of a microorganism by a progressive waving envelope (Blake, 1971). Finally, several approaches have addressed the question of the swimming velocity and rate of dissipation of motile microorganisms with different shapes. Analytically tractable examples of these biological microswimmers consist of point particles connected by active links exerting periodic forcing (Najafi and Golestanian, 2004; Felderhof, 2006), spherical particles that self-propel due to shape modulation of their surface (Felderhof and Jones, 2014), assemblies of rigid spheres that interact through elastic forces (Felderhof, 2014b), or one large sphere propelled by a chain of three little spheres through hydrodynamic and elastic interactions (Felderhof, 2014a). The latter configuration, in particular, has been realized and studied using optically trapped particles (Leoni et al., 2009).

\section{E. Artificial microswimmers}

Various methods have been developed to realize artificial microswimmers that can reproduce the swimming behavior of motile biological cells making use of diverse propulsion mechanisms. As seen in Sec. I, these man-made selfpropelling particles in fact hold the great promise to change the way in which we perform several tasks in, e.g., health care and environmental applications (Nelson, Kaliakatsos, and Abbott, 2010; Wang and Gao, 2012; Patra et al., 2013; Abdelmohsen et al., 2014; Gao and Wang, 2014). See 
Table I and Fig. 1 for examples of experimentally realized active particles.

The basic idea behind the self-propulsion of microparticles and nanoparticles is that breaking their symmetry leads to propulsion through various phoretic mechanisms. Independent of the specific propulsion mechanism, the absence of inertial effects requires nonreciprocal driving patterns in Newtonian liquids (Purcell, 1977). Indeed, the temporal motion of flagella or beating cilia found in biological systems follows nonreciprocal and periodic patterns (see also Sec. III.A). Accordingly, this also needs to be taken into account in the design of synthetic microswimmers.

The first demonstration of the concept of swimming powered by asymmetrical chemical reactions can be traced back to the seminal work by Whitesides and co-workers who made millimeter-scale chemically powered surface swimmers (Ismagilov et al., 2002), while the pioneering demonstration of microswimming in bulk was done by Paxton et al. (2004), who reported the propulsion of conducting nanorod devices. Subsequently, in an attempt to realize a synthetic flagellum, Dreyfus et al. (2005) fabricated a linear flexible chain of colloidal magnetic particles linked by short DNA segments. Such chains align and oscillate with an external rotating magnetic field and closely resemble the beating pattern of flagella; moreover, the strength of their swimming speed can be controlled by the rotation frequency of the field.

In general, microswimmers can be powered by two main categories of propulsion mechanisms (Ebbens and Howse, 2010): they can be powered by local conversion of energy (e.g., catalytic processes) or they can be driven by external (e.g., electric, magnetic, acoustic) fields. In this context, it is now important to remark that a distinction exists between internally driven active matter and particles that are brought out of equilibrium by external fields: while microswimmers powered by these two mechanisms feature a motion that can be described with similar effective models (see, e.g., Secs. II.A and II.B), they present quite different microscopic details in their interaction with their environment (see, e.g., their hydrodynamic properties discussed in Sec. III). In some cases, a combination of both is possible, e.g., an external field may be required to induce local energy conversion.

In this section, we first discuss the main physical principles of the propulsion mechanisms based on local energy conversion (Sec. II.E.1) and external fields (Sec. II.E.2). We then introduce the main experimental methods that are used to build a very successful class of artificial microswimmers, i.e., Janus particles (Sec. II.E.3).

\section{Propulsion by local energy conversion}

A versatile method to impose propulsion forces onto colloidal particles is the use of phoretic transport due to the generation of chemical, electrostatic, or thermal field gradients. When such gradients are generated externally, passive colloidal particles move of phoretic motion: for example, when colloids are exposed to an electrolyte concentration gradient, they migrate toward the higher salt regions (Ebel, Anderson, and Prieve, 1988). Therefore, if a particle generates its own local gradient, a self-phoretic motion can take place (Golestanian, Liverpool, and Ajdari, 2007).
The self-generation of gradients by a particle requires some type of asymmetry in its properties, e.g., its shape, material, or chemical functionalization. Based on such considerations, first Paxton et al. (2004) and then Fournier-Bidoz et al. (2005) observed that gold-platinum (Au-Pt) and gold-nickel (Au-Ni) microrods displayed considerably enhanced directed motion in hydrogen peroxide $\left(\mathrm{H}_{2} \mathrm{O}_{2}\right)$ solutions. An electrokinetic model seems to be consistent with most experimental observations: the bimetallic microrod is considered as an electrochemical cell that supports an internal electrical current in order to maintain a redox reaction at its two extremities, where protons are created ( $\mathrm{Pt} / \mathrm{Ni}$ end) and consumed (Au end). Due to the flux of protons along the rod, a fluid flow is generated that moves the rod. We remark that other mechanisms have also been suggested to explain the motion of these microrods, including the formation of oxygen bubbles (Ismagilov et al., 2002). This, however, would suggest the motion of $\mathrm{Au}-\mathrm{Pt}$ microrods to be in the direction of the Au end (i.e., opposite to the site where the oxygen bubbles are created), which is in disagreement with experimental observations.

Bubble formation in $\mathrm{H}_{2} \mathrm{O}_{2}$ aqueous solutions as the dominant driving mechanism has been observed in tubular structures of catalytic materials. The internal catalytic wall of these microjets (consisting of $\mathrm{Pt}$ ) decomposes $\mathrm{H}_{2} \mathrm{O}_{2}$ into $\mathrm{H}_{2} \mathrm{O}$ and $\mathrm{O}_{2}$. The produced $\mathrm{O}_{2}$ accumulates inside the tube and forms gas bubbles, which are ejected from one tube extremity, thus causing the propulsion of the microjet in the opposite direction (Solovev et al., 2009, 2010).

Biologically active swimmers have also been created by functionalizing a conductive fiber with glucose oxidase and bilirubin oxidase: in the presence of glucose, a redox reaction takes place leading to a proton flux and thus to a bioelectrochemical self-propulsion (Mano and Heller, 2005).

In contrast to electrically conductive systems, which are essential for the above driving mechanisms, propulsion can also be achieved with dielectric particles (e.g., made of silica, polystyrene, or melamine). The majority of such systems is based on so-called Janus particles (named after the two-faced Roman god), where dielectric colloids are partially coated with thin layers of catalytic materials like Pt or palladium (Pd) (Golestanian, Liverpool, and Ajdari, 2005). When such particles are immersed in an aqueous solution enriched with $\mathrm{H}_{2} \mathrm{O}_{2}$, they locally decompose it into $\mathrm{H}_{2} \mathrm{O}$ and $\mathrm{O}_{2}$, and thus create a local concentration gradient that eventually leads to self-diffusiophoresis. This concept, which was originally pioneered by Howse et al. (2007), has been very successful and has been used and modified by many other groups worldwide. Instead of Pt or Pd, hematite has also been used as a catalyst; this has the advantage of permitting one to control the $\mathrm{H}_{2} \mathrm{O}_{2}$ decomposition using light: in fact the hematite catalyzes the $\mathrm{H}_{2} \mathrm{O}_{2}$ decomposition only when illuminated with blue light (Palacci et al., 2013). The details of the catalytic processes involved in the $\mathrm{H}_{2} \mathrm{O}_{2}$ decomposition are quite complex and subject to current investigation. For example, the propulsion strength and direction show a strong dependence on added salt and ionic surfactants (Brown and Poon, 2014).

When metal-coated Janus particles are illuminated with strong laser light, temperature gradients along the particles can also form due to the selective heating of the metallic cap. This 
leads to a strong self-thermophoretic motion that can be controlled by the incident laser power, as it has been shown in the case of Au-capped colloidal particles (Jiang et al., 2009; Jiang, Yoshinaga, and Sano, 2010; Bregulla, Yang, and Cichos, 2013). However, the need for high intensity gradients can also lead to optical forces (Jones, Maragò, and Volpe, 2015), which can interfere with the self-diffusionphoresis mechanisms.

In contrast to thermophoretic effects, which require sufficiently strong light intensity, much smaller intensity is required when Janus particles are immersed in binary liquid mixtures with a lower critical point. When the bath temperature is kept sufficiently close to the critical temperature, even at small illumination intensities, light absorption at the cap leads to local heating, which results in a local phase separation and in a diffusiophoretic motion due to a concentration gradient across the particle (Volpe et al., 2011; Buttinoni et al., 2012). Because of the much smaller light intensities (compared to the thermophoretic mechanisms described earlier), optical forces are typically negligible. This propulsion mechanism has recently been theoretically investigated by Samin and van Roij (2015) and Würger (2015).

In addition to phoretic forces, Marangoni stresses can also generate particle propulsion. Experimentally, this has been demonstrated, e.g., in a system of water droplets (containing bromine) suspended in an oil phase and stabilized by a surfactant (Thutupalli, Seemann, and Herminghaus, 2011; Schmitt and Stark, 2013). Because of the spontaneous reaction of bromide with the surfactant (bromination), a self-sustained bromination gradient along the drop surface is generated that eventually leads to a Marangoni flow and thus propulsion. A similar mechanism has also been found in pure water droplets that are stabilized in an oil phase with surfactants above the critical micellar concentration (Izri et al., 2014). Capillary forces can also be exploited for self-propulsion through local heating produced by light absorption. Using this mechanism, asymmetric microgears, suspended at a liquid-air interface, can spin at hundreds rpm under wide field illumination with incoherent light (Maggi, Saglimbeni et al., 2015).

Finally, we remark that there are other driving mechanisms that do not rely on asymmetric particles, but where a spontaneous symmetry breaking occurs. For example, the reactive droplets studied by Thutupalli, Seemann, and Herminghaus (2011) produce a spontaneous symmetry breaking of the chemical reaction, which then determines the direction of the propulsion velocity. Izri et al. (2014) reported the spontaneous motion in a system consisting of pure water droplets in an oily surfactant medium. Bricard et al. $(2013,2015)$ demonstrated that self-propulsion can emerge as the result of a spontaneous symmetry breaking of the electric charge distribution around a colloidal particle immersed in a conducting fluid and in the presence of an electrical field.

\section{Propulsion by external fields}

In addition to mechanisms based on local energy conversion, a swimming motion can be achieved by the periodic nonreciprocal geometrical deformation or reorientation of the swimmer's body, which can be achieved by applying some (time-dependent) external fields that induce forces and mechanical torques on the object. For example, the use of a rotating magnetic field leads to the deformation of semiflexible rods (Dreyfus et al., 2005) and results in a movement resembling that of biological flagella (e.g., in bacteria and spermatozoa); a numerical study of this system was presented by Gauger and Stark (2006). In the case of rigid but chiral magnetic objects (propellers), a rotating magnetic field leads to the rotation of the propeller and thus to a swimming motion that can be fully controlled in three dimensions by using triaxial Helmholtz coils (Ghosh and Fischer, 2009). Alternatively, the application of vertical alternating magnetic fields to a dispersion of magnetic microparticles at a liquid-air interface leads to the formation of magnetic "snakes" due to the coupling between the liquid's surface deformation and the collective response of the particles (Snezhko et al., 2009). An elliptically polarized rotating magnetic field can generate the dynamic assembly of microscopic colloidal rotors, which, close to a confining plate, start moving because of the cooperative flow generated by the spinning particles acting as a hydrodynamic "conveyor belt" (Martinez-Pedrero et al., 2015; Martinez-Pedrero and Tierno, 2015).

Forces can also be applied to microscopic objects by excitation of ultrasound waves. Using the interaction of suspended objects with acoustic waves, the levitation, propulsion, rotation, and alignment of metallic microrods have been achieved (Wang et al., 2012): their directional motion is due to a self-acoustophoretic mechanism, whose driving strength depends sensitively on the shape asymmetry of the microrods (e.g., the curvature at their end).

Finally, the application of alternating electric fields to Janus particles has been predicted to lead to an unbalanced liquid flow due to induced-charge electro-osmosis (Ramos et al., 1998; Ajdari, 2000). Directed motion has indeed been observed when Au-coated colloidal particles in $\mathrm{NaCl}$ solutions are subject to uniform fields with frequencies in the range from $100 \mathrm{~Hz}$ to $10 \mathrm{kHz}$ (Gangwal et al., 2008).

\section{Synthesis of Janus particles}

Janus particles are special types of microparticles and nanoparticles whose surfaces have two or more distinct physical and/or chemical properties (Golestanian, Liverpool, and Ajdari, 2007). The simplest realization is achieved by dividing the particle into two distinct parts, each either made of a different material or bearing different functional groups. For example, a Janus particle may have one-half of its surface covered by hydrophilic groups and the other half by hydrophobic groups. This gives the particle unique properties related to its asymmetric structure and/or functionalization, which can, in particular, be exploited to obtain selfpropulsion. A recent comprehensive review by Walther and Müller (2013) covers all aspects from synthesis and selfassembly to novel physical properties and applications of Janus particles.

The synthesis of Janus nanoparticles requires the ability to selectively create each side of a particle with different chemical properties in a reliable, high-yield, and cost-effective way. Currently, three major methods are used (Lattuada and Hatton, 2011). 
Masking: The two main masking techniques that are commonly employed to produce Janus particles are evaporative deposition and suspension at the interface between two fluid phases (Lattuada and Hatton, 2011). In evaporative deposition (Love et al., 2002), homogeneous particles are placed on a surface (e.g., a cover slip) in such a way that only one hemisphere is exposed, typically forming a monolayer colloidal crystal. They are subsequently placed in an evaporation chamber, where they are covered on one side with some materials, typically a metal (e.g., $\mathrm{Al}, \mathrm{Au}$, or Pt) or carbon, and finally they are released in an aqueous solution (e.g., by sonication). In the phase-separation technique (Gu et al., 2005), the particles are placed at the interface between two phases in a liquid environment; subsequently, one side is exposed to a chemical that changes the particles' properties, and finally the Janus particles are released in a homogeneous solution. This latter technique scales particularly well to the nanoscale.

Self-assembly: This can be achieved by the use of block copolymers or selective absorption (Lattuada and Hatton, 2011). Block copolymers are made up of blocks of different polymerized monomers (Erhardt et al., 2001). Competitive absorption involves two substrates that phase separate due to one or more opposite physical or chemical properties. When these substrates are mixed with colloidal particles (e.g., Au nanoparticles), they maintain their separation and form two phases with the particles in the middle (Vilain et al., 2007).

Phase separation: This method involves the mixing of two or more incompatible substances that then separate into their own domains while still part of a single nanoparticle. This method can produce Janus nanoparticles made of two inorganic or organic substances (Carbone and Cozzoli, 2010; Lattuada and Hatton, 2011).

Nonspherical Janus particles with simple shapes, e.g., rods and cylinders, can be produced with methods similar to the ones discussed previously. For example, in the blockcopolymer method, the production of Janus spheres, cylinders, sheets, and ribbons is possible by adjusting the molecular weights of the blocks in the initial polymer and also the degree of cross-linking. More complex shapes can be obtained using microphotolithography, which has been used to produce the L-shaped particles shown in Figs. 3(c) and 3(d) (Kümmel et al., 2013), or glancing angle deposition, which has been used to produce the chiral colloidal propellers shown in Fig. 1(f) (Ghosh and Fischer, 2009).

\section{HYDRODYNAMICS}

As seen in Sec. II, active particles use a wide variety of mechanisms to achieve self-propulsion in liquid environments. In fact, often they are microswimmers that are capable of perturbing the surrounding fluid in a way that generates a net displacement of their own body. The structure of the dynamical laws governing fluid motion is therefore crucial to understand propulsion of individual swimmers. Furthermore, as an active particle moves in a fluid, a complex fluid flow pattern travels along with it and can influence the motion of nearby active or passive floating bodies. Moreover, whenever this flow pattern is distorted from its bulk structure by the presence of obstacles like confining walls, swimming speed, and direction can change leading to phenomena like swimming in circles or trapping by obstacles. The present section is structured in four main parts discussing hydrodynamic effects involved in generating propulsion in the bulk of a fluid (Sec. III.A), particle-particle hydrodynamic interactions (Sec. III.B), hydrodynamic couplings to confining walls (Sec. III.C), and swimming in a non-Newtonian medium (Sec. III.D).

\section{A. Microhydrodynamics of self-propulsion}

Swimming at the macroscopic scale heavily relies on the inertia of the surrounding fluid. For example, one of the most simple swimming mechanisms is that employed by scallops, which swim by opening and closing their two valves, as shown in Fig. 7. However, swimming strategies of this kind, i.e., involving a reciprocal shape deformation, are doomed to failure at microscopic scales (Purcell, 1977) (at least in purely viscous liquids, as seen in Sec. III.D), where fluid flows obey kinematic reversibility.

Slow flows of incompressible viscous fluids are governed by Stokes equations

$$
\eta \nabla^{2} \mathbf{u}-\nabla p=0, \quad \nabla \cdot \mathbf{u}=0,
$$

where $\eta$ is the fluid viscosity, $\mathbf{u}$ is the velocity of the flow, and $\nabla p$ is the gradient of the pressure. A flow is slow enough for Eqs. (15) to be valid when the typical speed $v \ll v_{\mathrm{c}}=\eta / \rho L$, where $\rho$ is the fluid density and $L$ is a characteristic length scale. The value of the characteristic speed $v_{\mathrm{c}}$ is on the order of $1 \mathrm{~m} \mathrm{~s}^{-1}$ for water when $L \sim 1 \mu \mathrm{m}$ and grows as the system size is reduced. The adimensional ratio between the two speeds is the Reynolds number

$$
\operatorname{Re}=\frac{v}{v_{\mathrm{c}}}=\frac{\rho L v}{\eta}
$$

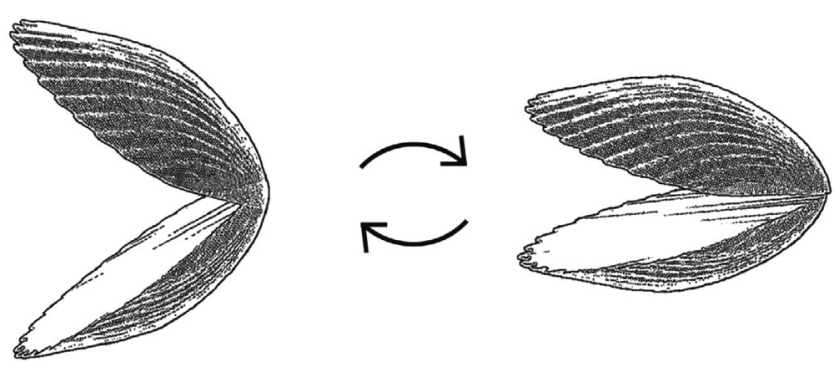

FIG. 7. The scallop theorem. Schematic drawing of a scallop performing a reciprocal motion: by quickly closing its two shells, the scallop ejects two fluid jets behind its hinge so that its body recoils in the opposite direction; then, by slowly opening the shells, the scallop comes back to its initial shape with little net displacement. The scallop theorem states that such a reciprocal motion cannot generate propulsion in a (viscous) fluid at a lowReynolds-number regime (Purcell, 1977). From Qiu et al., 2014. 
which is usually interpreted as the relative importance of inertial forces in comparison with viscous forces. Generally, $\operatorname{Re} \ll 1$ in all problems involving self-propulsion at the microscale, where typical flow speeds rarely exceed $100 \mu \mathrm{m} \mathrm{s}^{-1}$ (see Fig. 1 and Table I). Considering, for example, an $E$. coli bacterium swimming in water, $L \approx 1 \mu \mathrm{m}$, $v \approx 30 \mu \mathrm{m} \mathrm{s}^{-1}, \eta=0.001 \mathrm{Pas}$, and $\rho=1000 \mathrm{~kg} \mathrm{~m}^{-3}$, so that $\operatorname{Re} \approx 3 \times 10^{-5} \ll 1$.

A solid object that is dragged through the fluid by external forces imposes a boundary condition to the flow field given by the rigidity condition

$$
\mathbf{u}=\mathbf{v}+\omega \times \rho,
$$

where $\mathbf{v}$ and $\boldsymbol{\omega}$ are the instantaneous translational and rotational speeds of the object, and $\rho$ is a generic point on the body surface. The resulting flow and pressure fields can be obtained by solving Stokes equations [Eqs. (15)] with the boundary conditions [Eq. (17)]. By integrating the stress tensor $\boldsymbol{\sigma}=$ $\eta\left(\nabla \mathbf{u}+\nabla \mathbf{u}^{\mathrm{T}}\right)$ over the object's boundary, one obtains the following total force and torque acting on the body:

$$
\mathbf{F}=\oint_{S} \boldsymbol{\sigma} \cdot \hat{\mathbf{n}} d S
$$

and

$$
\mathbf{T}=\oint_{S} \boldsymbol{\rho} \times \boldsymbol{\sigma} \cdot \hat{\mathbf{n}} d S
$$

where $S$ is a surface enclosing the body, and $\hat{\mathbf{n}}$ is the unit vector perpendicular to the surface. Note that, as seen later, microswimmers are force-free and torque-free objects. From the linearity of Stokes equation the integrations result in a linear relationship

$$
\left[\begin{array}{c}
\mathbf{F} \\
\mathbf{T}
\end{array}\right]=\left[\begin{array}{cc}
\mathbf{A} & \mathbf{B} \\
\mathbf{B}^{\mathrm{T}} & \mathbf{D}
\end{array}\right]\left[\begin{array}{c}
\mathbf{v} \\
\boldsymbol{\omega}
\end{array}\right],
$$

where $\mathbf{A}, \mathbf{B}$, and $\mathbf{D}$ are tensors determined by the shape and orientation of the object. In particular, $\mathbf{A}$ represents the translational resistance tensor connecting the linear speed $\mathbf{v}$ to the force $\mathbf{F}$ that is needed to move a body in a purely translatory motion with speed $\mathbf{v}$. Similarly, $\mathbf{D}$ connects angular speed $\boldsymbol{\omega}$ to the torque $\mathbf{T}$ in pure rotations, and $\mathbf{B}$ and $\mathbf{B}^{\mathrm{T}}$ represent the coupling between rotational and translational motion. For example, for a sphere of radius $R, \mathbf{A}=6 \pi \eta R \rrbracket$, $\mathbb{D}=8 \pi \eta R^{3} \rrbracket$, and $\mathbf{B}=\mathbf{B}^{\mathrm{T}}=\mathbf{0}$. In the most general case, however, $\mathbf{A}$ and $\mathbf{D}$ are nonisotropic. Furthermore, for chiral bodies, i.e., bodies that do not possess three mutually orthogonal planes of symmetry, $\mathbf{B}$ and $\mathbf{B}^{\mathrm{T}}$ do not vanish. Therefore, a rotating chiral body can be used as a propeller generating a thrust force $\mathbf{B} \cdot \boldsymbol{\omega}$. The resistance tensor can be calculated analytically only in very few simple cases (Kim and Karrila, 2005). Approximation schemes with various degrees of complexity exist for slender bodies (Lighthill, 1976). Different schemes have been compared in the particular case of a helical propeller by Rodenborn et al. (2013). Direct experimental measurements of the resistance coefficients of a real flagellar bundle is possible by using optical tweezers (Chattopadhyay et al., 2006; Bianchi et al., 2015).

For example, swimming bacteria like E. coli use a helical bundle of flagellar filaments as a propeller for the cell body. The whole cell can be thought of as composed of two rigid bodies that translate as a single rigid object but can rotate with different speeds around a common axis. Assuming that the only nonvanishing speed components are those lying on the cell axis, we can write the following two scalar resistance equations for the cell body and the flagellar bundle:

$$
F_{\mathrm{b}}=A_{\mathrm{b}} v, \quad F_{\mathrm{f}}=A_{\mathrm{f}} v+B_{\mathrm{f}} \omega_{\mathrm{f}},
$$

where subscripts " $b$ " and " $f$ " refer, respectively, to cell body or flagellar bundle. Since no external forces are applied on the swimmer, $F_{\mathrm{b}}+F_{\mathrm{f}}=0$ and the swimming speed is obtained as

$$
v=\frac{B_{\mathrm{f}} \omega_{\mathrm{f}}}{A_{\mathrm{b}}+A_{\mathrm{f}}} .
$$

Imposing the torque-free condition one can similarly obtain the body rotation frequency.

The simplified picture of a microswimmer composed of two counterrotating rigid units holds only for swimming procaryotes like bacteria whose flagella are passive filaments connected to the cell body via a rotary motor (Berg, 2003). More generally, the swimming problem can be formulated starting from the common feature that active particles perturb the flow field by imposing specific boundary conditions for $\mathbf{u}(\boldsymbol{\rho})$, where $\boldsymbol{\rho}$ is the position vector of a generic point on the particle surface (Lauga and Powers, 2009). Self-propelling particles use two main mechanisms for imposing boundary conditions for the flow field over their surface:

(1) They can modify their shape and displace fluid at contact with sticky boundary conditions (swimmers).

(2) They can produce a gradient in some thermodynamic quantity resulting in a phoretic slip velocity that is tangential to the particle surface (squirmers).

The first strategy is mainly adopted by biological active particles that swim by waving or rotating flagella. The second mechanism typically applies to artificial self-propelling particles, like Janus colloids, that use some form of energy stored in the environment to maintain a stationary local gradient of, e.g., concentration or temperature. In both cases the hydrodynamic swimming problem can be formulated in general as follows:

(1) Solve Stokes equations with prescribed boundary conditions for the flow field on the particle surface [Eq. (17)], obtaining $\mathbf{u}(\mathbf{r})$.

(2) Integrate the viscous stress over the particle surface to get the total force and torque acting on the particle [Eqs. (18) and (19)].

(3) Impose force-free $(\mathbf{F}=\mathbf{0})$ and torque-free $(\mathbf{T}=\mathbf{0})$ conditions and solve for the rigid speeds $\mathbf{v}$ and $\boldsymbol{\omega}$.

The self-propulsion speed $\mathbf{v}(t)$ at a given time $t$ is then a function of the instantaneous values of the boundary speed distribution $\mathbf{u}(\boldsymbol{\rho})$ evaluated at the same time $t$. While most squirmers impose a stationary slip velocity on their boundary 
resulting in a constant $\mathbf{v}$, all swimmers necessarily deform in a cyclic (but not reciprocal) way resulting in a time-varying propulsion speed that cycles with the period $T_{\mathrm{p}}$ of the shape deformation. It can be shown that, since Stokes equations are linear and do not have any explicit dependence on time, the total net displacement after a cycle, i.e., $\int_{0}^{T_{\mathrm{p}}} \mathbf{v}(t) d t$, vanishes if the shape deformation is reciprocal, i.e., if the cycle is closed by retracing the same sequence of shapes in reverse order (Lauga and Powers, 2009). Najafi and Golestanian (2004) proposed a simple one-dimensional low-Reynolds-number swimmer consisting of three spheres linked by rigid rods whose lengths can change periodically but not reciprocally. Recently, Bet et al. (2016) derived a generalized scallop theorem that allows one to optimize the speed, power, and efficiency of a swimmer by altering its geometry. As a consequence of the scallop theorem a micron-sized scallop cannot swim at low Reynolds number but only move back and forth with no net displacement. Hydrodynamic reversibility, however, breaks down in non-Newtonian fluids (see Sec. III.D) allowing self-propulsion with reciprocal deformations (Qiu et al., 2014).

\section{B. Particle-particle hydrodynamic interactions}

Both forced colloids and active particles generate a flow that obeys Stokes equations [Eqs. (15)] subject to the appropriate boundary conditions for the flow field. Although analytical solutions can be obtained only in some very simple cases, the structure of the far-field flows can be discussed on a general basis and, as seen in the following, can be used to anticipate, at least qualitatively, the peculiar interactions that active particles manifest between themselves or with external obstacles and confining walls.

Since Stokes equations are linear, flow fields can be conveniently expressed as the superposition of singular solutions (Pozrikidis, 1992). The dominant singularity for particles driven by external forces is the Stokes flow generated by a point force, also known as the Stokeslet. For a point force of magnitude $F$ and direction $\hat{\mathbf{e}}$ the Stokeslet flow at a position $\mathbf{r}=r \hat{\mathbf{r}}$ from the force origin is given by

$$
\mathbf{u}_{\mathrm{s}}(\mathbf{r})=\frac{F}{8 \pi \eta r}[(\hat{\mathbf{e}} \cdot \hat{\mathbf{r}}) \hat{\mathbf{r}}+\hat{\mathbf{e}}] .
$$

The corresponding field is presented in Fig. 8(a). The magnitude of a Stokeslet decays as the inverse distance from the force origin and describes with good approximation hydrodynamic couplings between externally driven colloidal particles (Meiners and Quake, 1999; Di Leonardo et al., 2007) up to very short interparticle distances. Importantly, active particles propelled by internal forces are instead force free so that the leading term cannot be a Stokeslet.

The next singularity is the Stokes dipole, which can be obtained from the Stokeslet by differentiation and represents the far-field flow generated by two nearby and opposite point forces. There is a close analogy with the multipolar expansion of electric fields, although in the hydrodynamic case the field sources are vector forces rather than scalar charges. Therefore, while electric dipoles are represented by vectors, force dipoles are instead tensors, which can be symmetric when the two point forces are parallel to their separation direction, or antisymmetric when they point in the opposite orthogonal direction to their separation direction. However, since selfpropelled particles need to be torque free, the antisymmetric part, that would correspond to a point torque, vanishes and the far-field flow is usually dominated by a symmetric force dipole where forces and separation direction are parallel with the unit vector $\hat{\mathbf{e}}$ :

$$
\mathbf{u}_{\mathrm{d}}(\mathbf{r})=\frac{P}{8 \pi \eta r^{2}}\left[3(\hat{\mathbf{e}} \cdot \hat{\mathbf{r}})^{2}-1\right] \hat{\mathbf{r}},
$$

where $P$ is the magnitude of the force dipole having the dimensions of force times length. In flagellated swimming bacteria like $E$. coli the dipole arises from having most of the drag on the cell body side and pushing the fluid along the swimming direction while an opposite force is generated on the fluid all along the rotating flagellar bundle. The resulting flow pattern, shown in Fig. 8(b), is purely radial and pushes fluid in the forward and backward directions, while a lateral inward flow guarantees mass conservation. Swimmers of this (a) Stokeslet

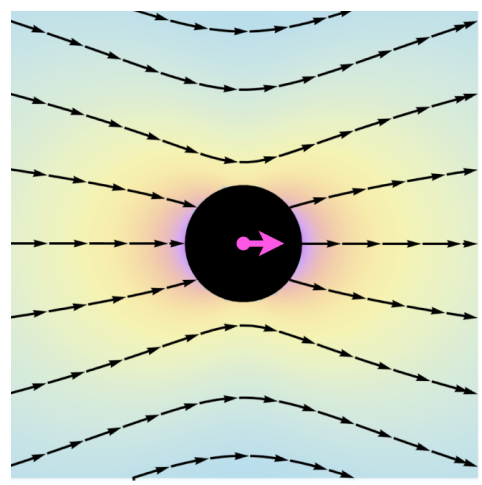

(b) Stokes dipole (pusher)

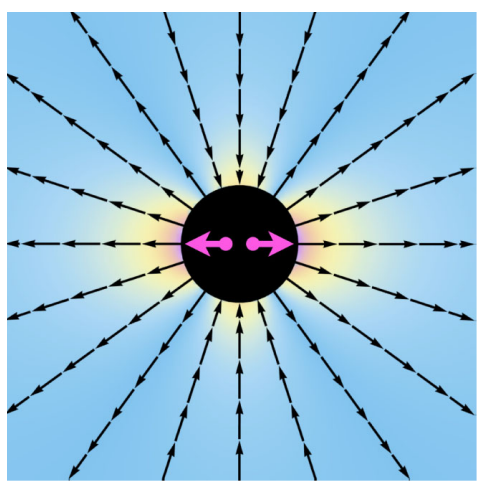

(c) Stokes dipole (puller)

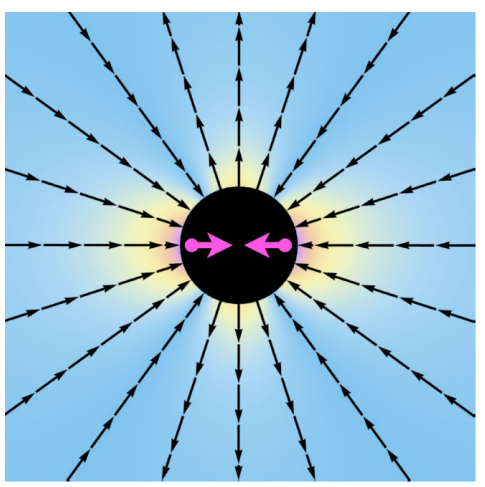

FIG. 8. Flow singularities. (a) Stokeslet corresponding to the far field of an active particle driven by an external force. (b), (c) Stokes dipoles corresponding to active particles driven by an internal force corresponding to (b) pushers and (c) pullers, both moving horizontally. 
kind are called pushers $(P>0)$ as opposed to pullers $(P<0)$ like the algae Chamydomonas reinhardtii, where two waving flagella propel a cell body that lags behind in the swimming direction [Fig. 8(c)]. ${ }^{4}$

Flows around swimming bacteria have been observed by microparticle imaging velocimetry (Drescher et al., 2011). The measured flow fields were well approximated by a dipole of Stokeslet with magnitude $F=0.4 \mathrm{pN}$ separated by a distance $d=2 \mu \mathrm{m}$. The magnitude of the force is consistent with the expected thrust force generated by the flagellar bundle, which is connected to the swimming speed $v$ by $F \approx \eta d v$. The generated flow field is approximately $v(d / r)^{2}$ representing a small perturbation over the free swimming speed $v$ of a nearby swimmer located at a distance $r$ larger than $d$. These perturbations could, however, manifest themselves as small couplings in the velocities of nearby swimming cells that are advected by the flows generated by neighbors. It was shown that, if one neglects correlations in the orientation of nearby swimmers, velocity couplings arise from quadrupolar terms, while the effect of dipolar flows cancels out on average (Liao et al., 2007). Baskaran and Marchetti (2009), starting with a minimal physical model of a stroke-averaged swimmer in a fluid, derived a continuum description of a suspension of active organisms that incorporates fluidmediated, long-range hydrodynamic interactions among the swimmers.

Dipolar flow fields also have an associated vorticity given by

$$
\Omega(\mathbf{r})=\nabla \times \mathbf{u}_{\mathrm{d}}=\frac{3 P}{4 \pi \eta r^{3}}(\hat{\mathbf{e}} \cdot \hat{\mathbf{r}})(\hat{\mathbf{e}} \times \hat{\mathbf{r}}) .
$$

Two nearby swimmers will therefore reorient each other through advection by the vorticity field produced by the neighbor. Since the vorticity field vanishes when either $\hat{\mathbf{e}} \cdot \hat{\mathbf{r}}=0$ or $\hat{\mathbf{e}} \times \hat{\mathbf{r}}=0$, two possible equilibrium relative orientations exist: the first one, where the swimming direction is orthogonal to the separation distance, is only stable for pushers $[P>0$, Figs. 9(a) and 9(c)]. The second one, with $\hat{\mathbf{e}}$ parallel to $\mathbf{r}$, becomes stable for pullers $[P<0$, Figs. 9(b) and 9(d)]. Interestingly, in both cases, the equilibrium relative orientation gives rise to reciprocally attractive flows [Eq. (24)]. The reorienting action exerted by nearby swimmers contributes to the bending of swimming trajectories. There are other mechanisms contributing to the reorientation of active particles, such as Brownian rotational diffusion, tumbles in flagellar locomotion, and cell-cell collisions through steric forces. The first two mechanisms do not depend on density and are always present even in diluted suspensions. In concentrated samples, however, cell-cell scattering, through either hydrodynamic interactions or direct contact, may become the dominant reorientation mechanism. Often, at those concentrations where hydrodynamic interactions become relevant, average interparticle distances are so small

\footnotetext{
${ }^{4}$ It has been shown experimentally that the flow field around Chamydomonas is not a simple dipole (Drescher et al., 2010; Guasto, Johnson, and Gollub, 2010).
}

(a)

(c)

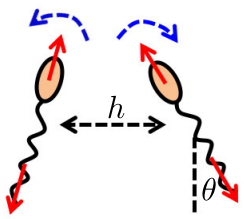

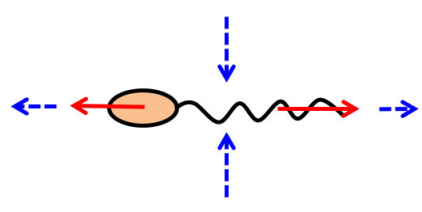

(b)

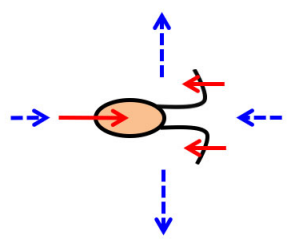

(d)

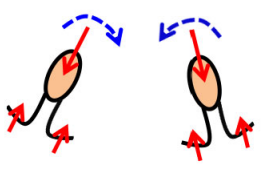

FIG. 9. Flow fields created by swimmers at low Reynolds numbers. (a) Pushers have a positive force dipole $(P>0)$ and induce an outgoing flow field directed along their swimming direction (repulsion) and an incoming flow field from their sides (attraction). In all panels, the solid red arrows represent the local forcing from the swimmer on the surrounding fluid and the dashed blue arrows represent the fluid flows around the swimmer. (b) Pullers have a negative force dipole $(P<0)$, inducing an incoming flow field along their swimming direction and an outgoing flow field along their sides. (c) Two pushers on a converging course reorient each other, tending toward a configuration where they are parallel and swimming side by side ( $h$ is the separation distance between the swimmers, and $\theta$ is the angle between the swimmers direction and the direction normal to their separation). (d) Two pullers on a diverging course reorient each other, tending toward a configuration in which they are antiparallel and swimming away from each other. From Lauga and Powers, 2009.

that higher-order singularities may become predominant (Liao et al., 2007; Drescher et al., 2011).

\section{Hydrodynamic coupling to walls}

Active particles may behave very differently when approaching a solid boundary. Although far-field hydrodynamics is often capable of anticipating the correct behavior (Spagnolie and Lauga, 2012), most reorientation dynamics take place when the particle is in close contact with the boundary. Near-field effects, steric interactions, and direct flagellar contact dynamics (Kantsler et al., 2013) become predominant in this regime giving rise to a broad range of behaviors, from stable wall trapping [Fig. 31(b)] to wall scattering (Fig. 10). Furthermore, active particles may exhibit rheotaxis (i.e., movement in response to a flow) near walls (Uspal et al., 2015a, 2015b).

Far-field predictions can be obtained considering the flow generated by the image singularities that need to be collocated inside a bounding wall in order to satisfy the no-slip boundary condition at the wall surface (Spagnolie and Lauga, 2012). In fact, the leading order effect of image singularities is qualitatively the same as that obtained by replacing the wall with a specular image swimmer located on the opposite side of the wall surface [see Figs. 9(c) and 9(d)]. Therefore, in a very similar way as discussed for swimmer-swimmer interactions, far-field reflected flows will align pushers in a direction that is parallel to the wall surface and attract them to the 
(a)

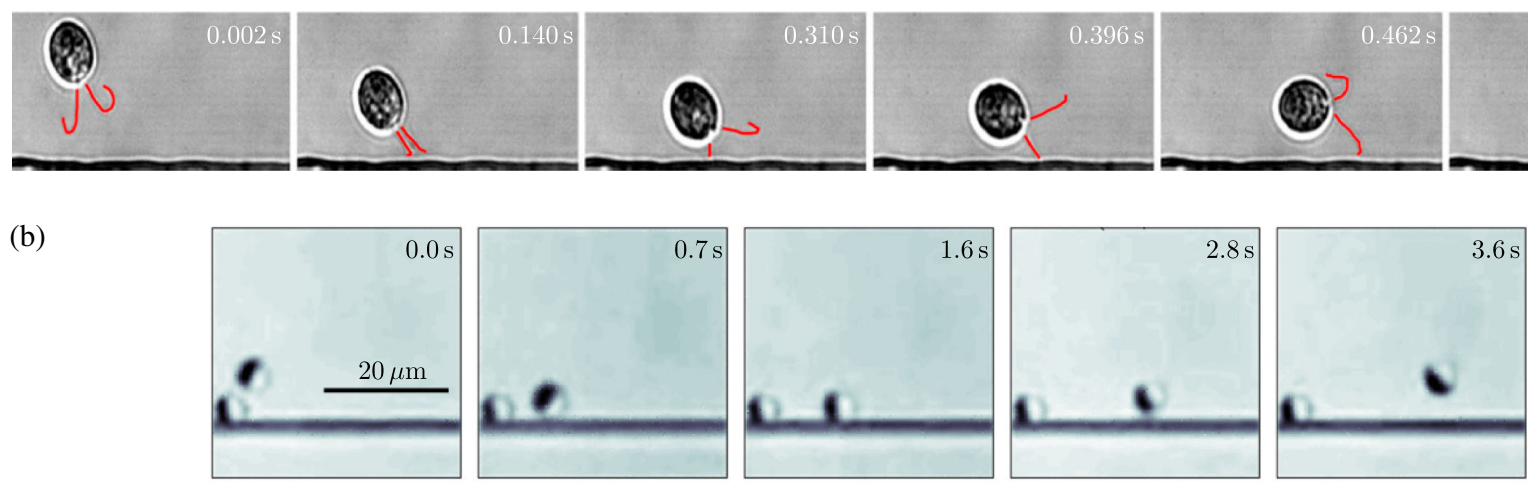

FIG. 10. Scattering of microswimmers by a wall: (a) the surface scattering of Chlamydomonas reinhardtii is governed by ciliary contact interactions (cilia manually marked in red). From Kantsler et al., 2013. (b) Time series of snapshots demonstrating the approach to, contact with, and detachment from a wall of a self-propelled Janus particle. From Volpe et al., 2011.

wall [Fig. 11(a)]. The stable swimming direction for pullers is parallel to the surface normal, leading again to an attractive image flow [Fig. 11(b)].

Although far-field predictions may correctly describe how active particles approach a wall, the actual collision dynamics and the fate of the resulting trajectory will depend on detailed near-field hydrodynamics and contact interactions. Swimming bacteria like E. coli display a remarkable tendency to swim in close contact with walls once they reach them (Frymier et al., 1995). Hydrodynamic effects have been proposed to be at the origin of this wall entrapment via two distinct mechanisms: the first one is via far-field reflected flows (Berke et al., 2008; Drescher et al., 2011) as discussed previously. The second mechanism involves hydrodynamic torques that arise in

(a)

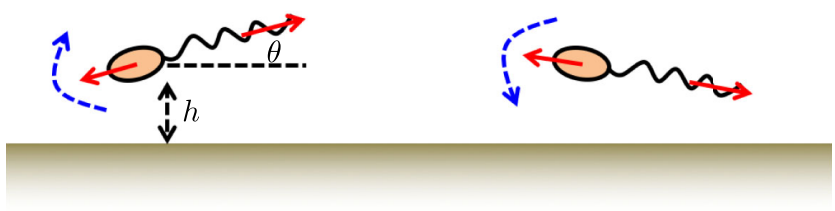

(b)
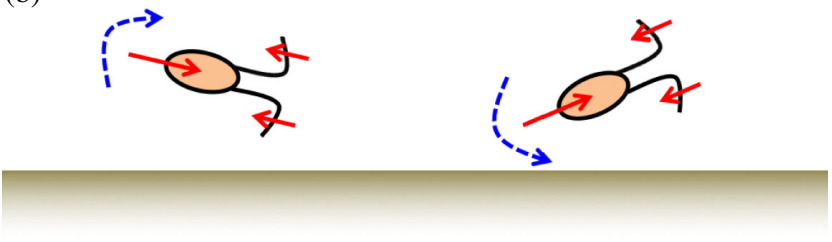

FIG. 11. Wall-induced rotation of microswimmers. A microswimmer is located at a distance $h$ from a solid surface and oriented at an angle $\theta$ with respect to the direction parallel to the surface: (a) pushers are reoriented hydrodynamically in the direction parallel to the surface [equilibrium $\theta=0$, see also Fig. 9(c)]; (b) pullers are reoriented in the direction perpendicular to the surface [equilibrium $\theta= \pm \pi / 2$, see also Fig. 9(d)]. The solid red arrows represent local forcing from the microswimmer on the surrounding fluid, and the dashed blue arrows represent the torque acting on the microswimmer. From Lauga and Powers, 2009. anisotropic bodies swimming in close contact with a wall and leading to tilted swimming, i.e., with the cell swimming direction pointing into the confining wall (Vigeant et al., 2002; Spagnolie and Lauga, 2012). It has been suggested that steric repulsion and rotational Brownian motion might be already enough to reproduce the observed accumulation of bacteria in the proximity of solid flat walls (Li and Tang, 2009; Volpe, Gigan, and Volpe, 2014). However, recent experiments have demonstrated that stable trapping is also observed around cylindrical pillars ruling out steric effects as the sole origin for wall entrapment in E. coli bacteria (Sipos et al., 2015). While pusher swimmers like E. coli mostly interact with the wall through the cell body that moves ahead of the flagella, puller swimmers like $C$. reinhardtii, moving with beating flagella ahead of the body, display more complex interaction dynamics with confining walls (Kantsler et al., 2013; Contino et al., 2015): near-field effects and direct ciliary contact lead to wall scattering, rather than wall trapping, with the cells escaping from the wall at a characteristic angle that does not depend on the initial direction of approach to the wall. Direct flagellar contact is also important in wall interactions for sperm cells due to the large amplitude of flagellar waveforms (Kantsler et al., 2013). In swimming bacteria like E. coli, the chiral nature of the flagella, when combined with the presence of a nearby wall, also results in a tendency to swim along clockwise circular trajectories above solid surfaces (Berg and Turner, 1990; Frymier et al., 1995; Lauga et al., 2006). Such a mechanism can be exploited to direct bacterial motions in microchannels (DiLuzio et al., 2005), or to design microfluidic devices that can sort bacteria according to their motility or size (Hulme et al., 2008). When the boundary condition on the wall changes from no slip on a solid wall to the almost perfect slip on a liquid-air interface, the direction of circular swimming is reversed (Di Leonardo et al., 2011).

\section{Non-Newtonian media}

Until now we have considered living microorganisms and artificial particles swimming in Newtonian liquids at low Reynolds numbers $(\operatorname{Re} \ll 1)$. However, most biological fluids, where also artificial microswimmers will be required to operate in future envisaged biomedical applications, are 
non-Newtonian (Lauga and Powers, 2009; Nelson and Peyer, 2014), e.g., blood, synovial, and cerebrospinal fluids, vitreous humour, mucus, and saliva (Fung, 1981; Nelson and Peyer, 2014). Despite this, propulsion of microswimmers in nonNewtonian fluids remains relatively unexplored.

Generally speaking, the physical properties of a fluid can be described by its viscosity $\eta$, which represents the following relationship between the shear stress $\tau_{\mathrm{s}}$ and the local shear velocity $\partial u / \partial y$ in a fluid along one direction $x$ :

$$
\tau_{\mathrm{s}}=\eta \frac{\partial u_{x}}{\partial y}
$$

where $u_{x}$ is the fluid velocity along the $x$ direction and the shear velocity is the gradient of the velocity along the perpendicular direction $y$. In a Newtonian fluid, $\eta$ is constant so that the viscous stresses arising from its flow, at every point, are linearly proportional to the local shear rates. NonNewtonian media, instead, are any fluids whose flow properties differ in any way from this case (i.e., where $\eta$ changes): the viscosity $\eta$ can change in different ways, and different types of non-Newtonian fluids can be defined, such as shearthinning and shear-thickening fluids where $\eta$ decreases or increases with the shear rate, as well as viscoelastic and viscoplastic fluids. For example, most of the fluids in the human body are viscoelastic (e.g., sputum, mucus, and vitreous humor) (Fung, 1981) with many of them featuring a shear-thinning behavior (e.g., saliva, blood, and synovial fluid) (Nelson and Peyer, 2014; Qiu et al., 2014). In the study of viscoelastic fluids, it is often useful to introduce the Weissenberg number, which is a dimensionless number that compares the viscous forces to the elastic forces; it is often given as

$$
\mathrm{Wi}=\dot{\gamma} \lambda,
$$

where $\dot{\gamma}$ is the shear rate and $\lambda$ is the stress relaxation time of the fluid.

The behavior of non-Newtonian media is different from that of simple Newtonian media, such as water, because of their very different microscopic organization, which typically includes the presence of molecules, microparticles, or other complex macromolecular structures. The motion of swimmers will, therefore, also be affected by the physical properties of the fluid in which they are placed: early experiments, for example, showed that $E$. coli and other types of bacteria can swim more efficiently in high-viscosity gel-forming fluids rather than in water (Schneider and Doetsch, 1974; Berg and Turner, 1979). The explanation of these experiments is still a matter of debate: the current standard model postulates the presence of bacteria-sized pores that allow them relative easy passage (Berg and Turner, 1979; Magariyama and Kudo, 2002). More recent studies suggest that the fast-rotating bacterial flagellum gives rise to a lower local viscosity in its vicinity (Martinez et al., 2014). In the low-Reynoldsnumber regime, Newtonian fluids are characterized by instantaneous and time-reversible flows that are described by the time-independent Stokes equations. As seen with the scallop theorem, a consequence of this fact is that a swimmer will not be able to move if it is just executing geometrically reciprocal motion (i.e., a sequence of changes in its shapes that are perfectly identical when time reversed) (Purcell, 1977): locomotion at low Reynolds numbers therefore generally requires nonreciprocal actuation of the swimmer that is achieved in nature, e.g., by breaking time-reversal symmetry with rotating helices (Turner, Ryu, and Berg, 2000) and with cilia that show flexible oarlike beats (Brokaw, 1965). However, theoretical and experimental work showed that breaking time-reversal symmetry is no longer a requirement in non-Newtonian fluids (Fu, Wolgemuth, and Powers, 2009; Keim, Garcia, and Arratia, 2012; Montenegro-Johnson, Smith, and Loghin, 2013; Qiu et al., 2014), where motion by periodic body-shape changes is possible when backward and forward strokes occur at different rates (MontenegroJohnson, Smith, and Loghin, 2013; Qiu et al., 2014). Since the scallop theorem no longer holds in complex non-Newtonian fluids, it is possible to design and build novel swimmers that specifically operate in these complex fluids: for example, fluid elasticity can be used to either enhance or retard propulsion in non-Newtonian fluids (Lauga, 2007; Leshansky, 2009; Teran, Fauci, and Shelley, 2010; Liu, Powers, and Breuer, 2011; Espinosa-Garcia, Lauga, and Zenit, 2013; Schamel et al., 2014). Qiu et al. (2014), in particular, reported a symmetric "microscallop" [Fig. 12(a)], a single-hinge microswimmer that can propel itself in a shear-thickening fluid by reciprocal

(a)

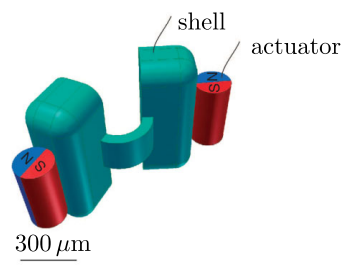

(b)

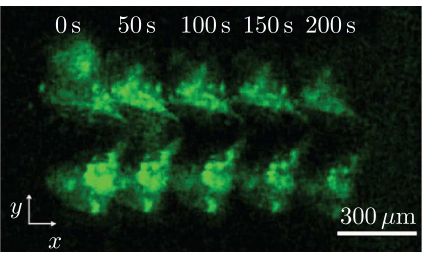

(c)
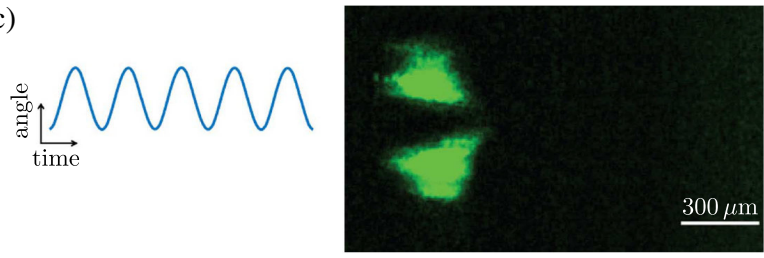

FIG. 12. Reciprocal swimming in a low-Reynolds-number nonNewtonian medium. (a) A 3D model of a submillimeter-sized "microscallop" capable of swimming in a low-Reynolds-number non-Newtonian fluid. (b), (c) Displacement of the microscallop in a shear-thickening and a shear-thinning fluid. (b) Forward net displacement of the microscallop in a shear-thickening fluid and asymmetric actuation (blue curve). The image is a time-lapse composite picture of five frames at intervals of $50 \mathrm{~s}$, with the net displacement occurring along the $x$ direction. (c) Corresponding image of the microscallop in shear-thickening fluid with symmetric actuation (blue curve) and no discernible net displacement. From Qiu et al., 2014. 
motion even at low Reynolds numbers when the activation is asymemtric [Fig. 12(b)], but not when it is symmetric [Fig. 12(c)].

\section{INTERACTING PARTICLES}

We now move toward the study of interacting active particles. This is both fundamentally interesting and important in terms of potential applications because various classes of active particles, such as bacteria and artificial microswimmers, are more realistically found in crowded environments, where they interact with both passive particles and other active particles. We first give an overview of the kinds of interactions that take place between colloidal particles, also introducing swarming models (Sec. IV.A). We then proceed to review two main aspects: we first discuss dense suspensions of active Brownian particles, where interesting transitions, such as clustering and self-jamming, can appear as a function of particle density or activity (Sec. IV.B). We then discuss the interactions between active and passive particles, where tantalizing phenomena, such as phase-separation and activedepletion forces, can emerge (Sec. IV.C).

\section{A. Classification of particle interactions}

As seen in Secs. II and III, the dynamical properties of isolated active particles are rather well understood and can be modeled in various ways, ranging from particle-based descriptions, where active particles can be assumed to be point particles, disks, ellipses, or otherwise-shaped objects that obey an overdamped equation of motion, to full continuum-based models, where hydrodynamic effects are explicitly taken into account. In all these models, the motion of a single active particle in a homogenous environment is ballistic at short times and diffusive at long times. However, the presence of other active particles in the surroundings leads to mutual interactions, which not only change the single-particle dynamics but also lead to the emergence of cooperative phenomena such as dynamic clustering or phase separation.

Active particles are often subject to the same interaction forces as particles at thermal equilibrium, but the final effects on the particles' dynamics can be strikingly different. We start by summarizing the most important effective interactions between passive colloids. First, colloidal stabilization can be achieved sterically, which is modeled by an excludedvolume interaction such that two colloids are treated as impenetrable objects that cannot overlap in space. Steric interactions are often numerically implemented in the following way: when a displacement makes two particles overlap, the particles are separated by moving each one-half the overlap distance along their center-to-center axis. In the numerical implementation of Brownian dynamics simulations, the hard-core interaction is typically softened to avoid discontinuities at overlap. Therefore soft repulsive interactions, such as a steep Yukawa model or a truncated-andshifted Lennard-Jones potential, are frequently employed to describe steric interactions. With the necessary adjustments, these considerations can be extended to particles with more complex shapes (Kirchhoff, Löwen, and Klein, 1996). Second, charge-stabilized suspensions are described by the traditional Derjaguin-Landau-Verwey-Overbeek (DLVO) theory (Derjaguin, 1941; Verwey, 1947), which involves a repulsive electrostatic part (often described by an effective pairwise screened Coulomb interaction) and an attractive part stemming from mutual van der Waals interactions. Other kinds of interactions can also emerge such as hydrodynamic interactions (discussed in Sec. III).

More complex models for the interactions can be considered for nonspherical particles by adding torques, bending effects, or rotations, which affect the direction of the propulsion or motor force (Vicsek and Zafeiris, 2012). Furthermore, multiple particles can be connected together by springs or other potentials to create active polymers or active membranes.

\section{Aligning interactions, Vicsek model, and swarming}

We now turn our attention to systems where there are interactions capable of aligning the motion of active particles. In practice, several types of interactions can lead to alignment. For example, alignment can be the result of hydrodynamic interactions between swimmers as seen in Sec. III.B and Fig. 9, steric interactions between elongated particles and even between self-propelled hard disks (Lam, Schindler, and Dauchot, 2015), and hydrodynamics and electrostatic between rolling colloids (Bricard et al., 2013).

Aligning interactions are particularly important in active matter systems, as they can lead to collective motion and swarming. One of the best-known and used collective motion models is the Vicsek model (Vicsek et al., 1995; Czirók, Stanley, and Vicsek, 1997). In its original version, the particles move with a constant velocity and interact only through an alignment term, whereby the direction of motion of particle $n$ is adjusted based on the average direction of motion of all neighboring particles within a flocking radius. A generalized version of the Vicsek model can be derived from the discretetime version of Eq. (4), which describes an active Brownian particle in two dimensions, by modifying the equation for the orientation of the particle, obtaining

$$
\begin{aligned}
x_{n}(t+\Delta t) & =x_{n}(t)+v \Delta t \cos \varphi_{n}+\sqrt{2 D_{\mathrm{T}} \Delta t} \xi_{x, n}, \\
y_{n}(t+\Delta t) & =y_{n}(t)+v \Delta t \sin \varphi_{n}+\sqrt{2 D_{\mathrm{T}} \Delta t} \xi_{y, n}, \\
\varphi_{n}(t+\Delta t) & =\left\langle\varphi_{m}(t)\right\rangle,
\end{aligned}
$$

where the averaging is over all the particles within a flocking radius. The Vicsek model exhibits a phase transition as a function of increasing particle density from undirected motion to a state where all the particles move in the same direction and bands of particles appear (Grégoire and Chaté, 2004; Chaté, Ginelli, Grégoire, and Raynaud, 2008; Ginelli and Chaté, 2010; Ramaswamy, 2010; Solon, Chaté, and Tailleur, 2015). Furthermore, the Vicsek model can be modified to include steric interactions between particles, which cause crystallization at high densities, as well as interactions with barriers or with a substrate (Drocco, Olson-Reichhardt, and Reichhardt, 2012). 


\section{B. Collective behaviors of active particles}

In this section we consider dense suspensions of active particles. First, we see how the presence of interacting active particles can lead to the formation of clusters, also known as "living crystals" (Sec. IV.B.1). Then, we see how the presence of active matter can change the rheological properties of the medium, inducing, in particular, active self-jamming (Sec. IV.B.2) and active turbulence (Sec. IV.B.3).

\section{Clustering and living crystals}

A dilute suspension of passive colloidal particles does not spontaneously form clusters unless there are strong attractive interactions. This is nevertheless possible in suspensions of active particles even in the presence of purely repulsive interactions. This was first predicted theoretically (Tailleur and Cates, 2008; Fily and Marchetti, 2012; Redner, Baskaran, and Hagan, 2013) and was recently observed experimentally (Buttinoni et al., 2013; Palacci et al., 2013).

A simple qualitative explanation of this phenomenon is shown in Figs. 13(a)-13(d): when two active particles collide, they block each other due to the persistence of their motion [sequence of Figs. 13(a) to 13(b)]. Such a two-particle cluster

(a)

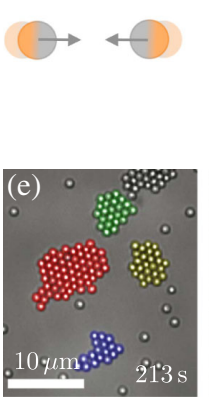

(i)

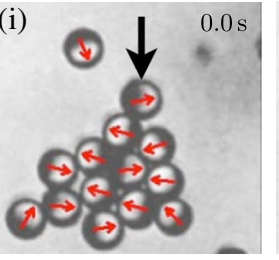

(b)

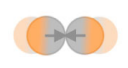

(c)

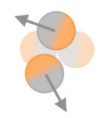

(d)
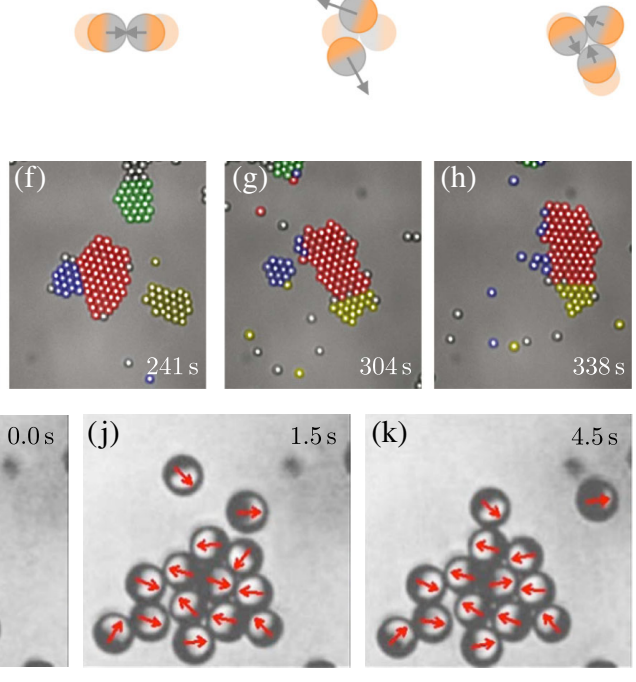

FIG. 13. Clustering and living crystals. Qualitative explanation of the clustering process: (a) when two active particles collide head on, (b) they block each other and form a two-particle cluster; (c) the cluster breaks apart on the time scale of the rotational diffusion; and (d) depending on the particle speed and density, another particle might collide before the two-particle cluster has broken apart and, thus, the cluster grows into a three-particle cluster. (e)-(h) Clusters assembled from a homogeneous distribution of active particles (solid area fraction $\phi=0.14$ ). The false colors show the time evolution of particles belonging to different clusters. The clusters are not static but rearrange, exchange particles, and merge. From Palacci et al., 2013. (i)-(k) Consecutive snapshots of a cluster of active Janus particles. The small dark gray (red) arrows indicate the projected orientations of the caps. Particles along the rim mostly point inward. The particle indicated by the large black arrow in (i) leaves the cluster (j) and is replaced by another particle (k). From Buttinoni et al., 2013. breaks when one of the two particles points away, which happens on a time scale comparable to the particle reorientation time [e.g., the rotational diffusion time $\tau_{\mathrm{R}}$ given by Eq. (2)] [Fig. 13(c)]. Since the mean time between collisions is controlled by the particle speed and density, depending on these parameters another particle might collide before the twoparticle cluster has broken, forming a three-particle cluster [Fig. 13(d)]. This leads to metastable clusters of a few particles or, if the mean collision time falls below a certain value, to the growth of ever larger clusters via the setting of a dynamical instability.

These qualitative considerations have in fact been confirmed by experiments where one observes the formation of clusters at intermediate particle densities (Theurkauff et al., 2012; Ginot et al., 2015) [see also the theoretical work by Pohl and Stark $(2014,2015)]$. Because of the steady particle collisions, these clusters are subjected to strong changes in size and shape with their average size increasing with the particle activity. Similar dynamic clusters of finite size were reported for hematite swimmers, as shown in Figs. 13(e)-13(h) (Palacci et al., 2013): in these experiments, the presence of attractive diffusiophoretic interactions could be directly measured. Comparison of the experiments with simulations suggested the presence of a diffusiophoretic aggregation mechanism which was caused by the interaction of the concentration profiles around each particle.

Experiments with active suspensions where diffusiophoretic interactions are negligible also show clustering and phase separation into dense clusters and a dilute gas phase at higher particle concentrations, as shown in Figs. 13(g)-13(k) (Buttinoni et al., 2013). These experimental observations were corroborated by numerical simulations of a minimal model, where only pure (short-ranged) repulsive interactions between the particles were considered and the behavior rationalized in terms of the self-trapping of active particles (Bialké, Löwen, and Speck, 2013).

The formation of active crystals was also observed as the result of collective dynamics of fast swimming bacteria (X. Chen et al., 2015; Petroff, Wu, and Libchaber, 2015).

Theoretical work has highlighted the connection between clustering and phase separation. Tailleur and Cates (2008) introduced the concept of motility-induced phase separation [for a recent review, see Cates and Tailleur (2015)]. Assuming a density dependent motility $v(\phi)$, there is an instability from a perturbed homogeneous suspension of swimmers if the gradient of $d v(\phi) / d \phi$ is sufficiently negative such that $[d v(\phi) / d \phi] / v(\phi)<-1 / \phi$. Microscopic approaches combined with an instability analysis start from the Smoluchowski equation (Bialké, Löwen, and Speck, 2013). For large length scales, clustering of active Brownian spheres can be mapped onto phase separation of a passive system with attractive interactions (Speck et al., 2014; Cates and Tailleur, 2015).

We finally remark that hydrodynamic near fields acting between squirmers can play a crucial role in determining their aggregation and collective motion (Zöttl and Stark, 2014). Interestingly, in the presence of a harmonic trapping potential, they can form a self-assembled fluid pump at large enough Péclet numbers (Hennes, Wolff, and Stark, 2014). 


\section{Self-jamming and active microrheology}

Jamming occurs when the density of a loose ensemble of particles such as grains or bubbles becomes high enough that the system can support a shear as if it were a solid. This phenomenon has been intensely studied in the contexts of granular matter, colloids, and emulsions (Van Hecke, 2010; Berthier, Jacquin, and Zamponi, 2011; Coulais, Behringer, and Dauchot, 2014). In certain cases jamming has been shown to have properties consistent with a phase transition (Liu and Nagel, 2010; Reichhardt and Olson-Reichhardt, 2014c). It is thus interesting to ask whether active matter systems can exhibit jamminglike features in the dense limit.

It might seem that increasing the activity would tend to suppress jamming. However, as seen in Sec. IV.B.1, active matter systems can form a self-clustering state in which the particles inside a cluster are locally jammed. Henkes, Fily, and Marchetti (2011) considered the active jamming of selfpropelled soft disks. They observed an aligned phase at low densities and a transition to a jammed phase for higher densities. Even within the jammed state, the activity induces large correlated motions of the particles. Berthier and Kurchan (2013) and Berthier (2014) analyzed the collective dynamics of self-propelled particles in the high-density regime, where passive particles undergo a kinetic arrest to an amorphous glassy state, and found that the critical density for dynamic arrest continuously shifts to higher densities with increasing activity.

One method for exploring the onset of jamming in a (passive or active) system is through microrheology. A probe particle is driven through a medium of other particles, and changes in the effective viscosity (or fluctuations of the probe motion) can be used to quantify changes in the medium (Squires and Brady, 2005; Candelier and Dauchot, 2009; Olson-Reichhardt and Reichhardt, 2010): if the medium is gaslike [Fig. 14(a)], the effective viscosity felt by the probe will be well defined with relatively small fluctuations, while if the particles in the medium are jammed [Fig. 14(b)], the effective viscosity will fluctuate more strongly and eventually acquire a dichotomic distribution (corresponding to motion
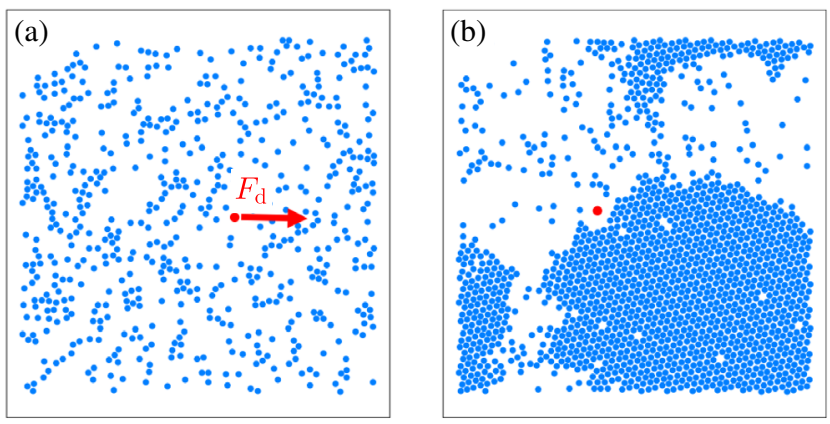

FIG. 14. Self-jamming. (a) Particle locations for an active matter system [dark gray (blue) particles] in a uniform liquid state at density $\phi \approx 0.2$ with an externally driven probe particle [light gray (red) particle]. The arrow indicates the direction of the probe driving force $F_{\mathrm{d}}$. (b) Phase-separated cluster state at $\phi \approx 0.5$, consisting of a high-density phase with local crystal ordering coexisting with a low-density liquid phase. From Reichhardt and Olson-Reichhardt, 2015. either in the gas or in the solid phase of the active particle solution).

Foffano et al. (2012) numerically studied a colloid driven through an active nematic bath, finding that the drag on the colloid was non-Stokesian and not proportional to the radius of the colloidal particle. They even observed instances of negative drag in which the particle moves in a direction opposite to an externally applied driving force.

Reichhardt and Olson-Reichhardt (2015) numerically studied a probe particle driven with a fixed external driving force $F_{\mathrm{d}}$ through a bath of run-and-tumble disks whose run length is fixed. At low densities $(\phi \approx 0.2)$, the active particles are in a liquid state [Fig. 14(a)], while as the density increases, there is a transition to a phase-separated or clustered state [Fig. 14(b) for $\phi=0.5$ ]. Previous studies of an active probe particle near a jamming transition also showed that the probe particle exhibits avalanche behaviors, which were argued to provide evidence that the jamming transition is a continuous phase transition with critical properties (Mognetti et al., 2013; Redner, Baskaran, and Hagan, 2013). In passive systems, jamming is associated with a specific critical density $\phi_{\mathrm{c}}$, while in active systems the clustered states self-organize into locally jammed regions with local density $\phi_{c}$, indicating that active systems can exhibit critical fluctuations well below the bulk jamming density of passive systems.

Even though the results available until now are only numerical, it should be possible to translate these to experiments. For example, it is in principle experimentally feasible to study the drag on a driven probe particle placed in an active bath of swimming bacteria or active colloids as the bath conditions are varied.

\section{Active turbulence}

A particularly interesting manifestation of collective behavior in microscopic active matter systems is the emergence of turbulent motion with the continuous formation and decay of whirls, jets, and vortices in dense solutions of active particles (Mendelson et al., 1999; Dombrowski et al., 2004; Riedel, Kruse, and Howard, 2005; Cisneros et al., 2007; Saintillan and Shelley, 2007; Sokolov et al., 2007; Breier et al., 2014). The observation of active turbulent patterns in a microscopic active system can be traced back to the experimental work of Mendelson et al. (1999), who observed the organization of swimming cells of Bacillus subtilis into short-lived dynamic patterns. The formation of these patterns seems to be ubiquitous among active matter systems since similar active turbulence has been reported in other active systems on widely varying length and time scales, from suspensions of microtubules, filaments, and molecular motors (Schaller et al., 2010; Sanchez et al., 2012; Sumino et al., 2012) to topological defects in nematic vesicles (Keber et al., 2014), vortices in active nematics (Giomi, 2015), agitated granular matter (Narayan, Ramaswamy, and Menon, 2007), and schools of fish and flocks of birds (Vicsek and Zafeiris, 2012).

Normally turbulence is a consequence of inertia, which is usually negligible at micrometric and smaller length scales; hence, the active turbulence seen in the microscopic active systems needs a different explanation, although, to date, a unified description of the formation and structure of these 
patterns remains lacking (Ishikawa et al., 2011; Wensink et al., 2012; Dunkel et al., 2013). The full characterization of the active turbulent spectra needs first a clear justification of power-law scaling over several decades and the corresponding derivation of new classes of nonuniversal exponents; an important step forward in this direction was recently done by Bratanov, Jenko, and Frey (2015).

\section{Mixtures of active and passive particles}

In this section we consider the interaction between passive and active particles. We start by considering the effect that relatively few active particles can have on the properties of a solution of passive particles (Sec. IV.C.1). We then consider the case of a mixture with similar numbers of active and passive particles, where one can observe phase separation and turbulent behaviors (Sec. IV.C.2). Afterward, we consider the limit where a few passive particles are immersed in a dense suspension of active particles: we therefore introduce the concept of active bath (Sec. IV.C.3), active-particle-powered directed motion and gears (Sec. IV.C.4), and active depletion and active-depletion forces (Sec. IV.C.5). Finally, we consider the role of the shape and flexibility of the passive particles on the effects of an active bath (Sec. IV.C.6). Interesting reviews on some aspects of the topics covered in this section can be found in Bialké, Speck, and Löwen (2015), Cates and Tailleur (2015), and Elgeti, Winkler, and Gompper (2015).

\section{Active doping}

Doping of colloidal suspensions with a very small amount of active particles can strongly influence their properties and dynamics.

Ni, Cohen Stuart, and Dijkstra (2013) and Ni et al. (2014) showed with numerical simulations that the crystallization of hard-sphere glasses can be dramatically promoted by doping the system with small amounts of active particles.

Kümmel et al. (2015) demonstrated with experiments and numerical simulations that the structure and dynamics of a suspension of passive particles is strongly altered by adding a very small $(<1 \%)$ number of active particles. Figure 15 illustrates the typical temporal changes in a colloidal suspension when doped with active particles; the active particles herd the passive particles favoring the formation of metastable clusters. Above a minimum passive particle concentration, it is possible to observe the formation of isolated dynamic clusters of passive colloids, which are surrounded by active particles. At higher passive particle concentrations, such activityinduced clusters start to merge and undergo further compression. When exceeding the threshold for spontaneous crystallization, active particles are found to accumulate at the interfacial regions between crystalline domains, where they lead to surface melting.

Using numerical simulations, van der Meer, Filion, and Dijkstra (2016) showed that active dopants can provide a route to removing grain boundaries in polycrystals: since, as we have seen, active dopants both generate and are attracted to defects, such as vacancies and interstitials, they tend to cluster at grain boundaries; thus, the active particles both broaden and enhance the mobility of the grain boundaries, causing rapid coarsening of the crystal domains. Finally, the remaining defects can be made to recrystallize by turning off the activity of the dopants, resulting in a large-scale single-domain crystal.

\section{Phase separation and turbulent behavior}

A few numerical studies looked into the behavior of dense mixtures with approximately comparable numbers of both active and passive particles. These investigations have reported the emergence of interesting novel phenomena including active-passive segregation between rodlike particles (McCandlish, Baskaran, and Hagan, 2012), emergence of flocking and turbulence (Hinz et al., 2014), and phase separation (Yang et al., 2012; Das et al., 2014; Yang, Manning, and Marchetti, 2014; Farage, Krinninger, and Brader, 2015; Stenhammar et al., 2015; Takatori and Brady, 2015; Tung et al., 2016).

$\mathrm{Su}$, Wang, and I (2015) performed a numerical study of self-propelled rods interacting with passive particles where the passive particles also interact with each other via a Yukawa repulsion that can be tuned from weak to strong. When the coupling between the passive particles is weak, they feature a turbulent behavior, while a strong coupling suppresses the turbulent behavior and allows the formation of a nearly triangular lattice.

\section{Active baths}

A passive particle is in an active bath when it is in an environment where a wealth of active particles are present, e.g., motile bacteria, as shown in Fig. 16(a) (Wu and
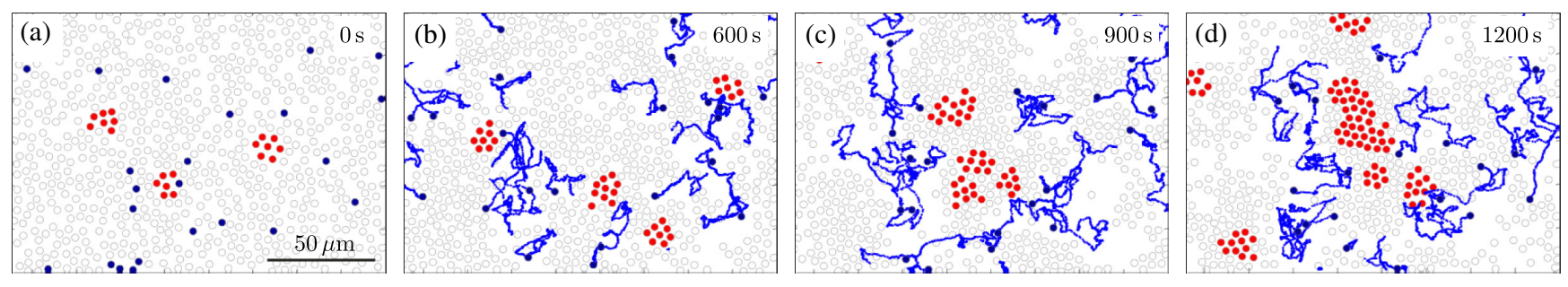

FIG. 15. Doping of a passive particle solution with active particles. Experimental snapshots of the temporal evolution of a mixture of passive $\left(\phi_{\mathrm{p}}=0.40\right)$ and active $\left(\phi_{\mathrm{a}} \approx 0.01\right)$ particles at (a) 0 , (b) 600, (c) 900, and (d) $1200 \mathrm{~s}$ for Péclet number Pe $\approx 20$. The passive particles belonging to clusters are represented as light gray (red) circles, while those not belonging to clusters are represented as open circles. Active particles are shown as dark gray (blue) circles and their trajectories over $300 \mathrm{~s}$ prior to each snapshot are represented as solid lines. From Kümmel et al., 2015. 
(a)

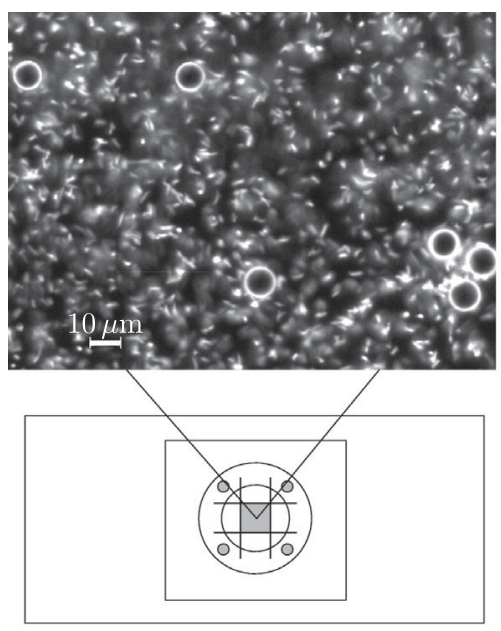

(b)

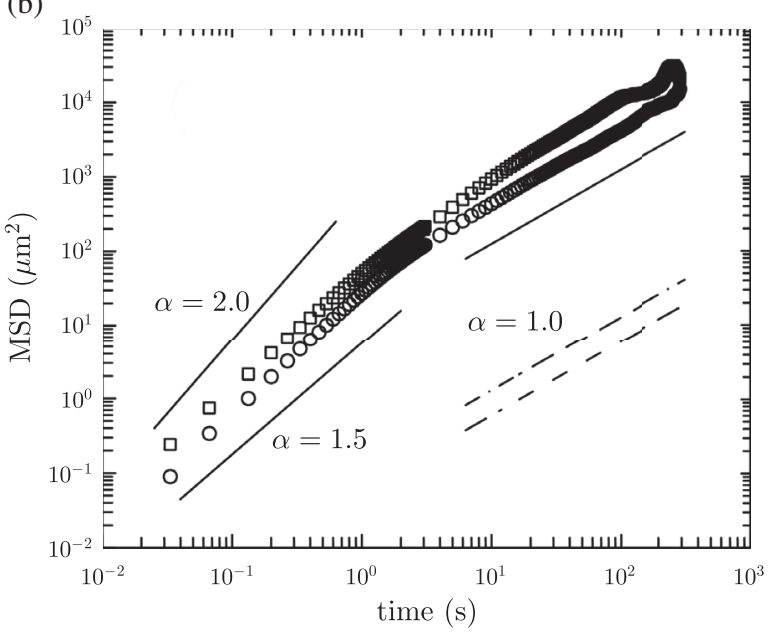

FIG. 16. Superdiffusion of passive particles in an active bath. (a) Experimental setup and fluorescence image of a solution with E. coli mixed with $10-\mu$ m polystyrene spheres. (b) MSD measurements of polystyrene spheres with diameters 4.5 (squares) and $10 \mu \mathrm{m}$ (circles) at a particle concentration of $\approx 10^{-3} \mu^{-2}$ in an active bath. The solid lines with slopes $\alpha=2.0,1.5$, and 1.0 are guides to the eyes. The two dashed lines correspond to the thermal diffusion of $4.5-\mu \mathrm{m}$ and $10-\mu \mathrm{m}$ particles. From Wu and Libchaber, 2000.

Libchaber, 2000). These particles will exert nonthermal forces on the passive object so that it will experience nonthermal fluctuations and will behave widely different from a passive Brownian particle in a thermal bath. For example, a passive colloidal particle in a dense bacterial bath behaves like an active particle due to multiple interactions with the selfpropelled bacteria: the measured mean square displacements indicate superdiffusion at short times and normal diffusion at long times (Wu and Libchaber, 2000), as shown in Fig. 16(b). However, the characteristic time at which this transition occurs is not related to the rotational diffusion, but rather to the density and activity of the bacteria acting as active particles. The long-time diffusivity of passive tracers can be orders of magnitude larger than their thermal counterparts ( $\mathrm{Wu}$ and Libchaber, 2000; Miño et al., 2011) and also displays exponential tails in the distribution of displacements (Leptos et al., 2009; Lin, Thiffeault, and Childress, 2011). Active particles in the bath can contribute to the enhanced diffusivity of passive tracers through both hydrodynamic interactions (Grégoire, Chaté, and Tu, 2001; Thiffeault and Childress, 2010; Lin, Thiffeault, and Childress, 2011; Pushkin and Yeomans, 2013, 2014) and direct contact forces (Grégoire, Chaté, and Tu, 2001; Valeriani et al., 2011).

The presence of an active bath can also significantly influence the microscopic thermodynamics of a particle. For example, Argun et al. (2016) considered the applicability of Jarzynski equality in the presence of an active bath, finding that it fails because of the presence of non-Boltzmann statistics. This observation points toward a new direction in the study of nonequilibrium statistical physics and stochastic thermodynamics, where also the environment is far from equilibrium.

Moving a step farther, it is also interesting to consider the interactions that emerge between passive particles in an active bath. Angelani et al. (2011) conducted simulations and experiments of passive particles immersed in a bath of runand-tumble swimming bacteria. Figure 17(a) shows a snapshot of the simulation where the bacteria are represented as spherocylinders with white-colored "heads" indicating their direction of swimming and the passive particles are modeled as spheres. Figure 17(b) shows a corresponding experimental snapshot. As the level of activity of the bacterial bath increases, a short-range "attraction" emerges between passive particles as evidenced by a pronounced peak in the radial distribution function in Fig. 17(c). Figure 17(d) indicates the radial distribution function obtained in experiment, in good agreement with the simulation. The origin of this shortrange attraction is a nonequilibrium effect arising from the persistent character of the fluctuating forces exerted by bacteria.

\section{Directed motion and gears}

Differently from spherical particles, asymmetric passive particles placed in an active bath can feature a directed motion. For example, we consider the case of a V-shaped wedge, such as the one shown in Fig. 18. If the wedge is surrounded by passive particles [Fig. 18(a)], there is no directed motion, because the pressure due to the passive particles is equal on all sides of the object. However, in an active bath, the active particles accumulate in the cusp of the wedge, producing its net propulsion [Fig. 18(b)]. This simple model system has been studied by various groups both numerically and experimentally (Angelani and Di Leonardo, 2010; Mallory, Valeriani, and Cacciuto, 2014; Wensink et al., 2014; Smallenburg and Löwen, 2015). Angelani and Di Leonardo (2010) employed asymmetric wedges to create bacteriapowered shuttles. Kaiser et al. (2014) performed experiments and simulations of a passive wedge placed in a bacterial bath and found that the wedge can undergo directed motion. Maximal efficiency occurs for an intermediate bacterial density, so that a turbulent bath maximizes the speed of the carrier. This can be qualitatively understood by considering two limits: for high dilution, the pushing effect is small since only few swimmers are contributing. For high density, the bath 
(a)

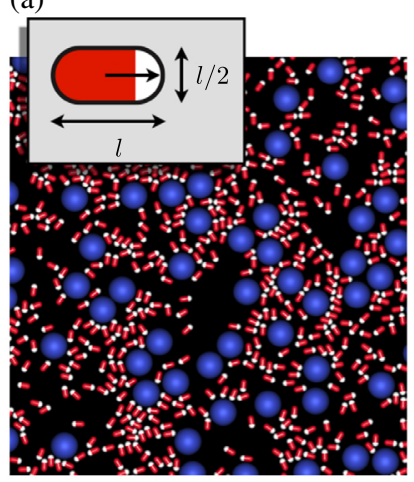

(b)

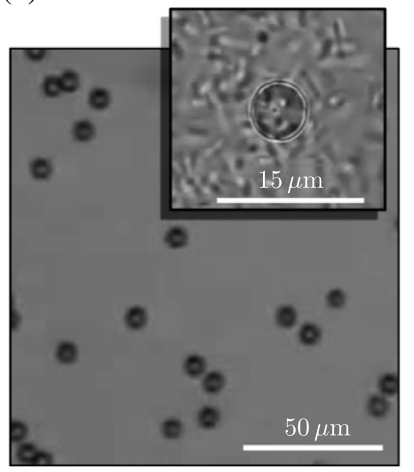

(c)

(d)

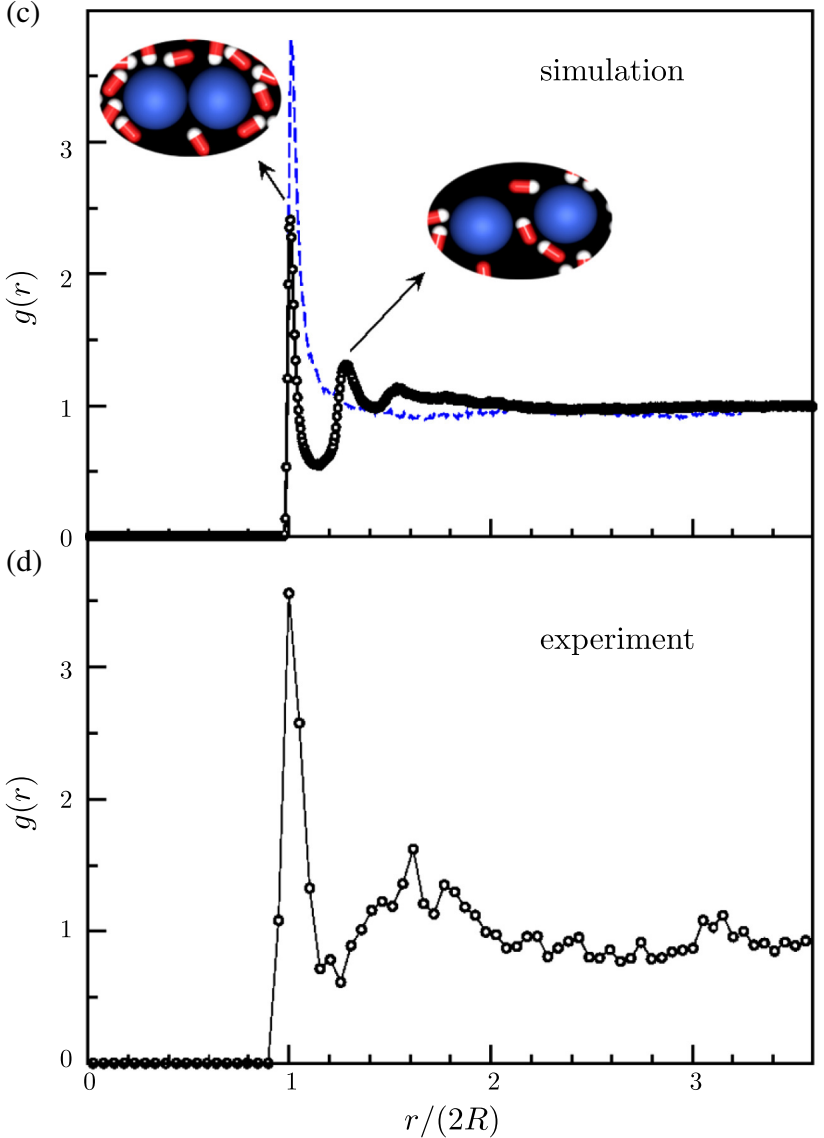

FIG. 17. Interactions mediated by an active bath. (a) Snapshot of a simulation of passive (spherical) particles immersed in an active bath. The active particles are motile bacteria (represented by spherocylinders with white heads pointing in the direction of selfpropulsion). (b) Microscopy image from experiment. The bacteria are visible in the enlarged image in the inset. (c) Simulated and (d) experimental radial distribution functions $g(r)$. Typical two-bead configurations corresponding to the first two peaks are also shown in the insets in (c). Distances are in units of particle diameter $2 R$ (bacteria width is about 0.25 in such a unit). The dashed line is the $g(r)$ obtained in simulations with a mixture of bacteria interacting with only one of the particles, i.e., without depletion. From Angelani et al., 2011.

is jammed, which leaves no mobility for the carrier; hence, there must be an optimal density that maximizes the transport efficiency in the intermediate turbulent state. As a function of the wedge apex angle the carrier speed attains a maximum at (a)

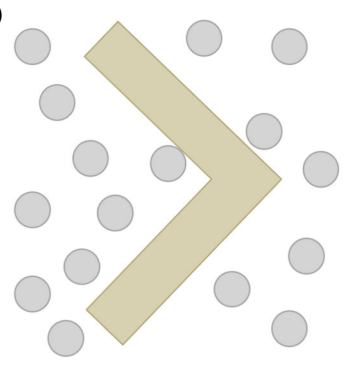

(b)

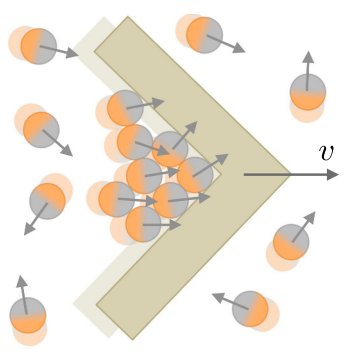

FIG. 18. Motion of a passive wedge in an active bath. (a) If the wedge is surrounded by passive particles, there is no directed motion, because the pressure due to the passive particles is equal on all sides of the object. (b) However, in an active bath, the active particles accumulate in the corner of the wedge, producing its net propulsion.

about $90^{\circ}$ (Kaiser et al., 2014). Mallory, Valeriani, and Cacciuto (2014) numerically studied a system of asymmetric tracers (partial circles with varied curvature) placed in a bath of self-propelled Janus particles and found that even low densities of active particles can induce pronounced directed motion of the tracers. U-shaped particles were found to be optimal to obtain enhanced transport (Smallenburg and Löwen, 2015). Typically, the tracer speed increases with increasing persistence time of the individual self-propelled particles.

Going beyond a simple wedge, it is also possible to consider self-starting cogwheels that exhibit a spontaneous rotation when in an active bath (Angelani, Di Leonardo, and Ruocco, 2009; Di Leonardo et al., 2010; Sokolov et al., 2010). These bacterial-driven micromotors work as active matter ratchets, whose key operational feature is the trapping of the self-propelled particles in the funnel tips, against which the particles exert their motor force. Purely thermal particles will not accumulate in the funnel tips but will instead diffuse uniformly throughout the system. In the active matter case, the particles exert sufficient force on the cogwheel to move it, indicating that active matter can be used to perform work on appropriately shaped objects. Angelani, Di Leonardo, and Ruocco (2009) performed a numerical study where motile bacteria were modeled as rod-shaped particles undergoing run-and-tumble dynamics and large mobile objects in the shape of asymmetric gears were placed in the bacterial bath. Figures 19(a) and 19(b) illustrate the microscopic particlewall interactions responsible for the rotation of the gear: in Fig. 19(a), a particle enters the frame from the left, runs along the wall of the gear (outlined in gray), and is shunted back into the bulk. In Fig. 19(b), instead, a particle entering from the right is trapped at the corner of the gear and continues swimming into the corner, generating a torque that drives the rotation of the gear. Thus, the active particles collect in the inner corners of the gear teeth and produce a net torque on it, gradually rotating it in one direction as illustrated by the plot of the angular velocity $\omega$ versus time in Fig. 19(c), and producing a realization of a bacteria-powered motor. When a symmetric gear is used instead, as shown in the inset of Fig. 19(c), it does not rotate since the forces exerted on it by the active bath are symmetric. Di Leonardo et al. (2010) 
(a)

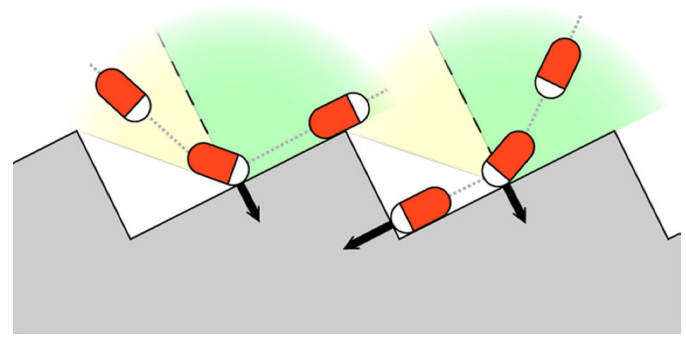

(c)

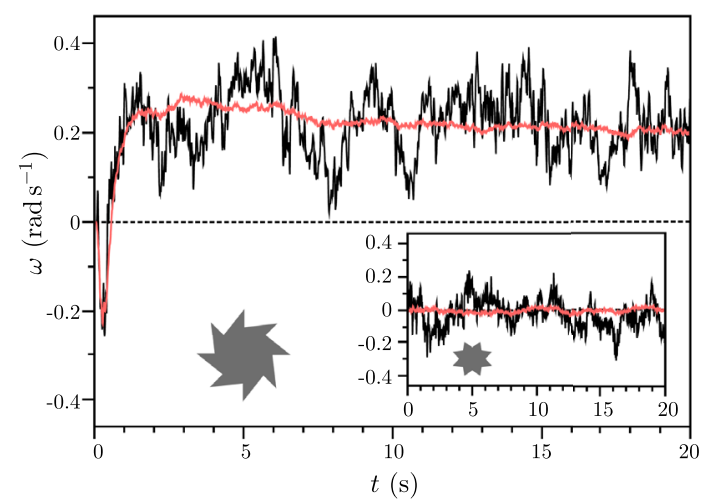

(d)

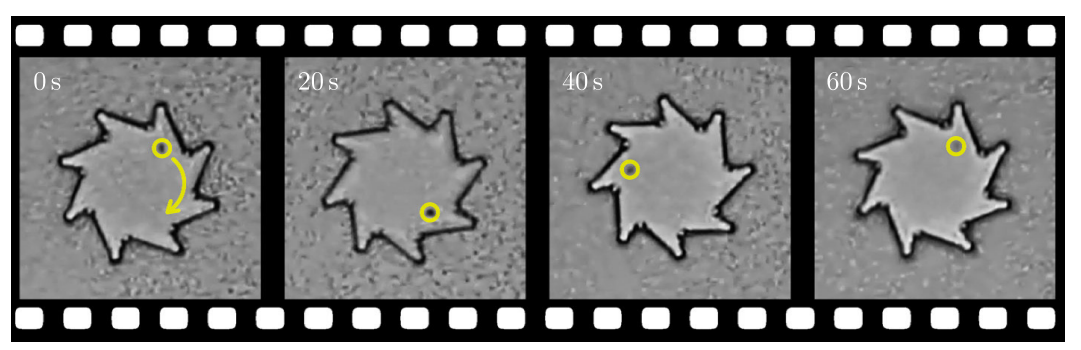

FIG. 19. Bacteria-driven micromotors. (a), (b) Sketch of the collision of a single bacterium with the rotor boundary: (a) bacteria coming from the left area with respect to the normal leave the gear, while (b) bacteria from the right get stuck at the corner exerting a torque on the rotor. The arrows represent the forces exerted by the bacteria on the rotor. (c) Angular velocity $\omega$ of the micromotor as a function of time: the black line refers to a single run; the red (lighter) line is the average over 100 independent runs. After a short transient regime (due to the initial configuration of bacteria), a fluctuating velocity around a mean value $\omega_{0} \approx 0.21 \mathrm{rad} \mathrm{s}^{-1}$ is observed. Inset: The same as the main plot for a symmetrically shaped micromotor, which does not rotate (on average). From Angelani, Di Leonardo, and Ruocco, 2009. (d) A nanofabricated asymmetric gear (48 $\mu$ m external diameter, $10 \mu$ m thickness) rotates clockwise at $1 \mathrm{rpm}$ when immersed in an active bath of motile $E$. coli cells, visible in the background. The gear is sedimented at a liquid-air interface to reduce friction. The circle points to a black spot on the gear that is used for visual angle tracking. From Di Leonardo et al., 2010.

confirmed these results experimentally as shown in Fig. 19(d), showing that an asymmetric gear rotates. Sokolov et al. (2010) also performed experiments on asymmetric gears placed in a bath of swimming bacteria and emphasized that the collective effect of many swimming bacteria can considerably enhance the rotation rate. At high bacteria concentrations, however, the motility of the bacteria decreases, causing a decrease in the rotation rate of the gear. Rectification of asymmetric gears has also been studied for the case where the active particles are small robots (Li and Zhang, 2013) or Janus particles (Maggi et al., 2016). In the latter case, it was shown that a perfectly ordered micromotor can self-assemble when the edge lengths of the microgear are properly chosen with respect to Janus particle diameter.

\section{Active depletion}

Going beyond the directed motion (or rotation) that an active bath can produce on a single passive object, it is also interesting to consider what kind of interactions emerge between multiple passive particles in the presence of an active bath. We can talk about active depletion, as this case is the natural generalization of the depletion forces arising in passive baths (Asakura and Oosawa, 1954).

Ray, Reichhardt, and Olson Reichhardt (2014) studied one of the simplest configurations, i.e., the case of two parallel plates surrounded by a bath of run-and-tumble active particles. As shown schematically in Fig. 20(a), the plates are separated by a distance $d$ and the net force acting on the plates can be measured as $d$ is varied. The active particles are not allowed to interact with each other but only with the walls, corresponding to a very low active particle density regime. ${ }^{5}$ Particles can enter the region only between the plates by approaching from the sides over a limited range of angles, which becomes more limited as $d$ decreases. In contrast, particles can reach the outer edges of the plates from any angle. This generates an effective active-depletion force that pushes the two plates together. It is also possible to exploit this shadowing effect by constructing very long walls with a very small aperture in order to preferentially trap particles between the two plates, resulting in a negative (i.e., repulsive) effective force. This opens the possibility that objects with carefully chosen geometries, when placed in an active bath, could experience a crossover from attractive to repulsive forces as their spacing $d$ diminishes, producing a tunable mimic of an interatomic potential.

${ }^{5}$ Ray, Reichhardt, and Olson Reichhardt (2014) also considered the effect of including particle-particle interactions in the dilute limit and found that the attraction between the plates persists but is reduced in magnitude. 

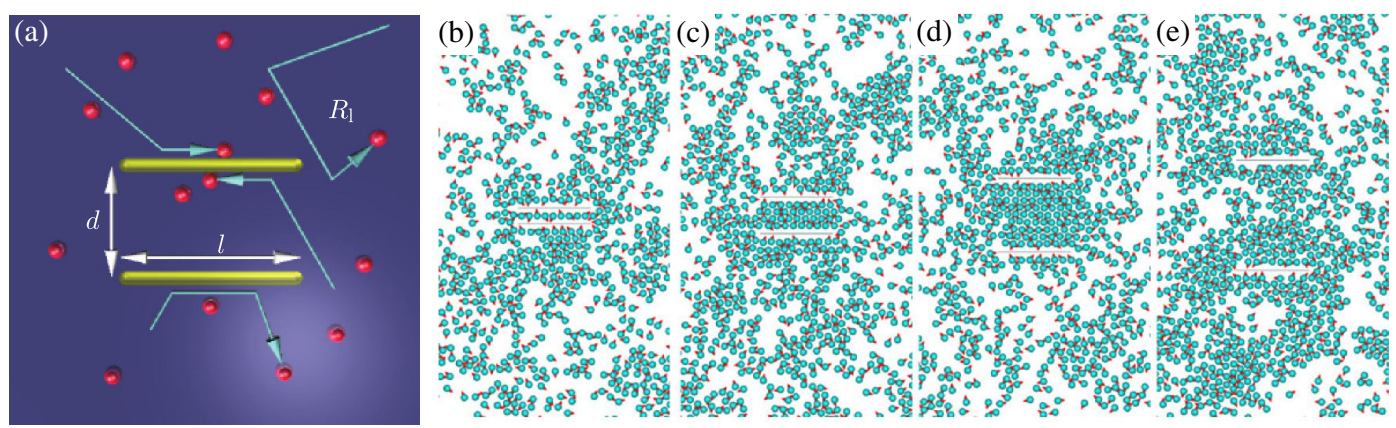

FIG. 20. Active-depletion interactions between two plates in an active bath. (a) A schematic of the system containing run-and-tumble particles (spheres) with some particle trajectories indicated by lines and arrows. The run length is $R_{1}$. The two parallel walls (bars) of length $l$ are separated by a distance $d$. When a particle moves along a wall, it imparts a force against the wall. From Ray, Reichhardt, and Olson Reichhardt, 2014. (b)-(e) Typical snapshots of systems for density distributions with a wall-to-wall distance $d / R$ equal to (b) 2.1 , (c) 5, (d) 10, and (e) 20, where $R$ is the radius of the active particles. If the particles are modeled as hard spheres, repulsive as well as attractive active-depletion forces can emerge. From Ni, Cohen Stuart, and Bolhuis, 2015.

$\mathrm{Ni}$, Cohen Stuart, and Bolhuis (2015) studied a similar geometry consisting of two plates and active particles modeled as repulsively interacting colloidal particles whose direction of motion undergoes a gradual rotational diffusion rather than sudden run-and-tumble changes. For a density of $\phi=0.4$, well below the passive crystallization density of $\phi=0.9$, they found that the force between the plates has an oscillatory nature, with a net repulsive force. This effect arises due to the formation of a bridge between the two plates composed of a densely packed, partially crystalline cluster of active particles, while in the bulk no clusters are present, as illustrated in Figs. 20(b)-20(e). As seen in Sec. IV.B.2, in interacting active matter systems without walls or obstacles, there is a transition from a liquid state to a phase-separated cluster state when the particle density or activity level is high enough. The walls act as nucleation sites for clusters of active particles even for particle densities well below the clean phase-separated regime. When the nucleated clusters grow large enough, they form a bridge between the two walls and the repulsive interactions between the particles in the cluster produce a net repulsive force between them. When the spacing $d$ between the plates is large enough, the bridge can no longer span the two plates and the net force between the plates is strongly reduced. Oscillations of the force arise from the crystalline ordering of the particles between the walls: due to the finite radius of the particles, certain values of $d$ are commensurate with integer numbers of particle diameters and at these spacings well-ordered crystals can form between the plates, producing a stronger effective plate-plate repulsion. Ni, Cohen Stuart, and Bolhuis (2015) also considered the dilute limit and found an exponential attractive interaction between the walls, similar to that obtained by Ray, Reichhardt, and Olson Reichhardt (2014). The magnitude and range of the force increase with increasing self-propulsion. Ni, Cohen Stuart, and Bolhuis (2015) also observed that the attraction arises due to a reduction of the particle density between the walls.

Similar effects have also been considered for two spherical objects immersed in an active bath (Das et al., 2014; Harder et al., 2014). Here the concept of swim pressure has turned out to be helpful (Takatori, Yan, and Brady, 2014; Smallenburg and Löwen, 2015; Solon, Fily et al., 2015; Solon, Stenhammar et al., 2015; Yan and Brady, 2015). Understanding the wall curvature dependence of the swim pressure gives access to a general theory of depletion in an active bath, so that the anaytical Asakura-Oosawa model for a thermal noninteracting bath can be appropriately generalized to the active case (Smallenburg and Löwen, 2015). For example, Harder et al. 2014)), using numerical simulations, considered the interactions between large objects immersed in an active bath, as shown in Fig. 21(a), and found that the shape of the objects plays an important role in determining the polarity of the force: as shown in Fig. 21(b), there is an attractive force between two interacting passive disks due to a depletion effect even when the active motor force is null (i.e., $F_{\mathrm{a}}=0$ ), while when the disks are active (i.e., $F_{\mathrm{a}} \neq 0$ ), the force between them becomes repulsive. In this case, the repulsion arises due to the accumulation of active particles in the corner regions between two closely spaced disks. The active particles generate a net outward force that pushes the disks apart. When the disks are inactive, the accumulation of active particles in the corners does not occur. When the large passive particles are rod shaped rather than disk shaped, there is an attractive force between the rods that increases with increasing activity. The case of parallel rods is then similar to the Casimir geometry in the dilute limit where attractive forces arise.

We remark that the basic notion of active depletion is that the presence of obstacles in an active bath causes guided motion of the active particles along the surface of the obstacles, leading to concentration gradients of the bath particles. By contrast, for passive depletion, the obstacles cause gradients by impeding the paths of the bath particles, once the obstacles get close to each other. Ray, Reichhardt, and Olson Reichhardt (2014) provided a clean demonstration of depletion which is purely active, with no passive component. In fact, the active particles have zero size and, therefore, passive depletion must play no role, because zerosize particles cannot be excluded by any obstacle.

There are also many other types of fluctuation-induced force effects that occur in active matter systems and can depend on the shape or flexibility of both the active and passive particles. For example, Parra-Rojas and Soto (2014) 
(a)

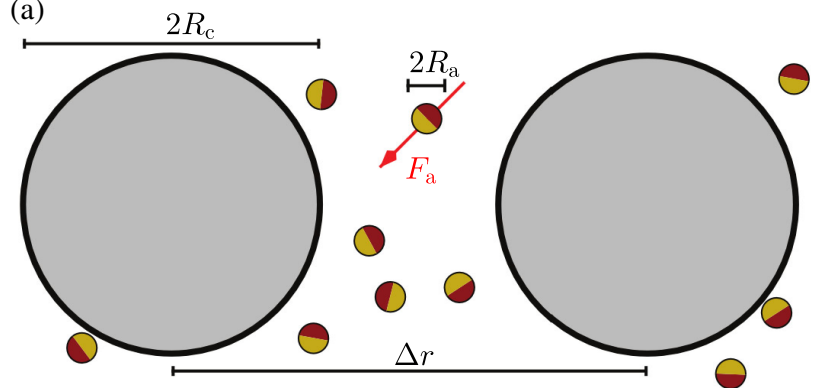

(b)

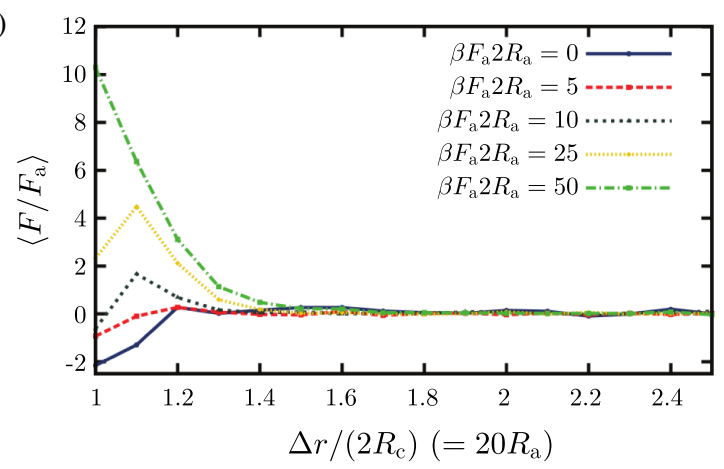

FIG. 21. Emergence of repulsive active-depletion forces in active baths. (a) Schematic representation of two colloidal disks in a bath of active particles. The persistent force $F_{\mathrm{a}}$ acts along a defined axis, which is shown by the arrow as well as the colors, where dark gray (red) corresponds to the back of the particle and light gray (yellow) to its front. (b) Effective rescaled forces $\left\langle F / F_{\mathrm{a}}\right\rangle$ experienced by two colloidal disks as a function of their separation for different values of depletant's activity for $R_{\mathrm{c}}=10 R_{\mathrm{a}}$, where $R_{\mathrm{c}}$ is the radius of the colloids and $R_{\mathrm{a}}$ is the radius of the active particles $(\phi=0.1)$. Rescaling is applied only as long as $\beta F_{\mathrm{a}} 2 R_{\mathrm{a}} \neq 0$. Positive values correspond to a repulsion, which dominates any depletion-driven interaction when the bath is active. The larger the active force and the larger the colloid-to-depletant size ratio, the stronger the repulsion. From Harder et al., 2014.

argued that genuine Casimir effects ${ }^{6}$ can occur in microswimmer suspensions since, due to the discreteness of the particles in suspension, the fluctuations in the system depend on the local particle density.

Adding active particles to an ensemble of passive particles could lead to new routes of rapid self-assembly or tunable selfassembly in which the structures could rapidly disassemble when the activity is reduced. Additionally, many biological systems may be exploiting active fluctuations to bring objects together, such as within or around cells (Machta, Veatch, and Sethna, 2012).

\section{Flexible passive particles and polymers}

The scaling behavior of polymers or chains under thermal fluctuations has a rich history (de Gennes, 1979). Methods for

\footnotetext{
${ }^{6}$ When two plates are placed in some form of fluctuating environment, confinement effects can produce an attractive force between them that is known as the Casimir effect (Casimir, 1948; Lamoreaux, 1997; Munday, Capasso, and Parsegian, 2009; Intravaia et al., 2013). As a function of the spacing $d$ between the plates, Casimir forces typically obey a power law $F_{\mathrm{C}} \propto d^{-\alpha}$, where $\alpha>3$.
}

characterizing polymers include Flory scaling exponents in which the extension $S$ of a polymer goes as $S \propto N_{\mathrm{m}}^{\nu}$, where $N_{\mathrm{m}}$ is the number of units along the chain (or the molecular weight) and $\nu$ is the Flory exponent. Since polymers, chains, and elongated structures are common in biological systems, it is interesting to ask how polymers behave in the presence of an active bath rather than a thermal bath.

Kaiser and Löwen (2014) performed two-dimensional simulations of a flexible polymer chain in a bacterial bath in a relatively diluted limit and found that for very long chains the polymer extension follows a two-dimensional Flory scaling. This occurs since the chains are considerably longer than the bacterial running lengths, so the polymers sample the bacterial bath fluctuations only at long time scales, at which the bacteria effectively undergo regular diffusion. When the chains are short, i.e., of the order of the bacterial running length or smaller, the activity becomes important and the chains are expanded or compressed by the bacterial bath.

Harder, Valeriani, and Cacciuto (2014) also performed numerical studies of a chain in an active bath and confirmed the results of de Gennes (1979) in the fully flexible limit. They then added rigidity to the chains and observed a variety of new types of behaviors, including a crumbling transition to a metastable hairpin structure at intermediate activity levels. This is illustrated in Fig. 22, which shows fluctuations between a hairpin configuration and an elongated configuration of the polymer. Protein folding has been a long-standing problem and is generally understood to occur under thermal fluctuation conditions; however, the results of Harder,

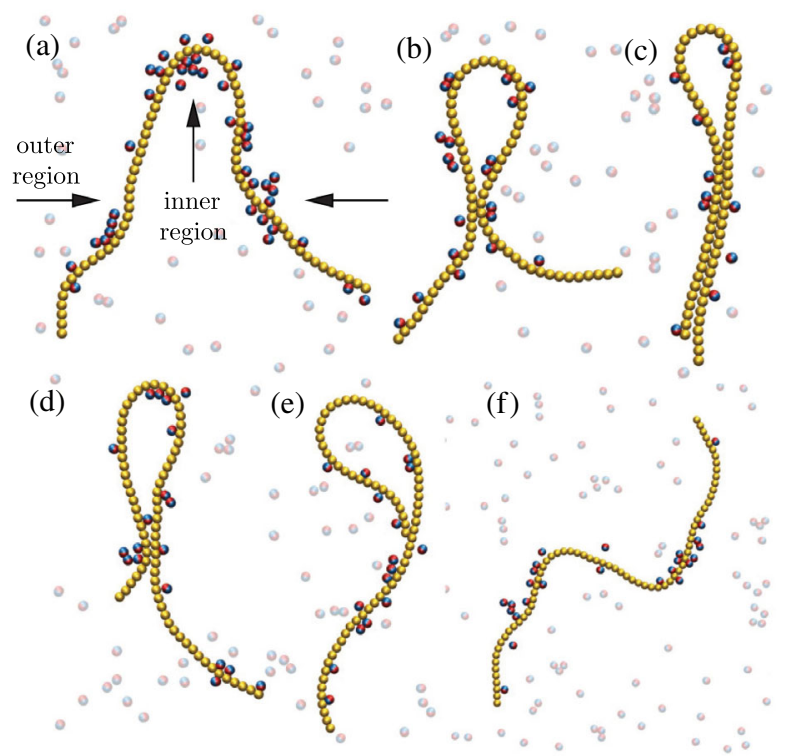

FIG. 22. Flexible chain in an active bath. (a)-(f) Sequence of snapshots from simulations depicting a filament at various stages of folding and unfolding. The propelling force acts along the axis connecting the poles of the two hemispheres used to depict the active particles in the light-to-dark gray (blue-to-red) direction. For the sake of clarity, active particles away from the filament have been rendered with a semitransparent filter. In (a), the convention for the definition of the inner and outer regions of a bent filament are explicitly shown. From Harder, Valeriani, and Cacciuto, 2014. 
Valeriani, and Cacciuto (2014) indicate that folding configurations may be facilitated by the presence of active fluctuations. This suggests that active matter dynamics could play an important role in certain biological functions such as the folding of chains or membranes (Suzuki et al., 2015). Therefore, this is an important direction for future study in active matter systems (Isele-Holder, Elgeti, and Gompper, 2015; Mallory, Valeriani, and Cacciuto, 2015; Shin et al., 2015).

Kaiser et al. (2015) numerically and analytically studied the case of a single chain composed of active particles in the absence of any bath, either thermal or active. They considered a free chain, a chain confined by an external trap, and a chain being dragged by one end, and observed Flory exponents of $\nu=0.5,0$, and 1 , respectively. Here the activity does not change the Flory exponents but it does modify the prefactor of the scaling law. When self-avoidance is added to the model, the equilibrium Flory exponents still appear, but the chain extension becomes nonmonotonic for increasing activity.

\section{COMPLEX ENVIRONMENTS}

So far we have considered only individual and collective behaviors of active particles moving in homogeneous environments without any physical boundaries. Another avenue of research in active matter systems is the study of the interaction of active particles with physical obstacles and boundaries. On the one hand, such situations are relevant for biological microswimmers moving in many natural habitats, from soil and the guts to culture media such as agar (Berg, 2004). For example, it is a tantalizing possibility that motile biological systems might have evolved specific rules of motion that facilitate navigation through similar complex environments. On the other hand, understanding how to control the motion of artificial active particles through their interaction with complex environments could prove beneficial also for applications, e.g., inside lab-on-a-chip devices or living organisms (Chin, Linder, and Sia, 2007).

This section will therefore focus on the role of boundaries on the swimming properties of microswimmers and nanoswimmers. We first consider how microswimmers interact with a planar wall and explain the basic particle-obstacle interaction mechanisms as well as their modeling (Sec. V.A). We then consider active particles confined within a pore and show how their behavior differs from that of passive particles in similar confined environments (Sec. V.B). Next we consider the effects that emerge when microswimmers interact with various kinds of complex environments (Sec. V.C). Finally, we discuss how microswimmers can be sorted exploiting micropatterned environments (Sec. V.D).

\section{A. Interaction with a wall}

There are various ways in which a microswimmer can interact with an obstacle. The two most important are arguably hydrodynamic and steric interactions, even though other (e.g., electrostatic, depletion) interactions can also occur. The basic concepts have already been introduced in Sec. III for hydrodynamic effects, and in Sec. IV.A for other kinds of particleparticle interactions.
The simplest situation in which an active particle interacts with an object is arguably when it interacts with a planar (vertical) wall; some examples (where hydrodynamic interactions are important) are reproduced in Fig. 10. In this case, there is a basic asymmetry between approaching and leaving the wall: when approaching the boundary, the particle will remain effectively stuck at the wall until its orientation points away from it; when leaving, it will just swim away. This asymmetry leads to a tendency for swimmers to accumulate near confining boundaries, which is not observed for particles at thermodynamic equilibrium. In fact, even if the wall is purely repulsive, there is a large accumulation which would require large attraction strengths for passive particles at equilibrium. This effect has been observed for rods and spheres in a linear channel (Wensink and Löwen, 2008; Elgeti and Gompper, 2009; Volpe et al., 2011; Wysocki, Elgeti, and Gompper, 2015) as well as for bacteria in circular cavities (Vladescu et al., 2014). Chiral active particles, however, have been shown to behave differently, as they can slide along a planar wall using their effective driving torque (van Teeffelen and Löwen, 2008; van Teeffelen, Zimmermann, and Löwen, 2009).

Both steric and hydrodynamic interactions typically lead to an alignment of the active particle such that its self-propulsion is directed along the wall of the obstacle. Steric effects of linear (Wensink and Löwen, 2008) and circle swimmers (van Teeffelen and Löwen, 2008; van Teeffelen, Zimmermann, and Löwen, 2009; Kümmel et al., 2013) with a planar wall can be qualitatively understood using the effective force description introduced in Sec. II.B. When a self-propelled particle hits an obstacle such as a planar wall, the propelling force can be decomposed into two components: one tangential and one normal to the wall. The tangential component leads to sliding along the wall while the normal component is compensated by the steric wall-particle interaction. Numerically, this process can be modeled using reflective boundaries, as shown in Fig. 23 (Volpe, Gigan, and Volpe, 2014), even though more accurate higher-order algorithms are also available (Behringer and Eichhorn, 2011, 2012). Importantly, Elgeti and Gompper (2013) showed that these steric effects are sufficient to produce the accumulation and alignment of particles with a wall, without the need for hydrodynamic interactions. (a)

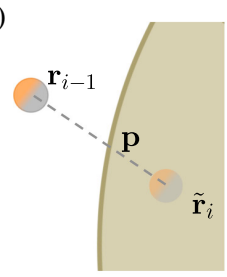

(b)

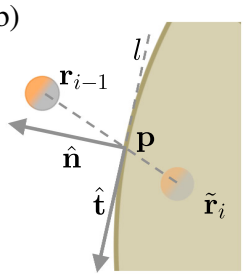

(c)

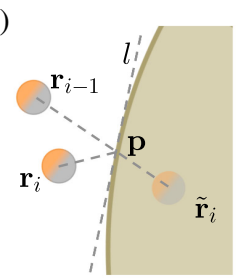

FIG. 23. Numerical implementation of reflective boundary conditions. At each time step, (a) the algorithm checks whether a particle has moved inside an obstacle. If this is the case, (b) the boundary of the obstacle is approximated by its tangent $l$ at the point $\mathbf{p}$ where the particle entered the obstacle and (c) the particle position is reflected on this line. In (b), $\hat{\mathbf{t}}$ and $\hat{\mathbf{n}}$ represent the tangential and normal unitary vectors to the surface at point $\mathbf{p}$. Note that the orientation of the particle is not flipped. 
Hydrodynamic interactions between the active particle and the wall are also an important kind of interaction (although frequently neglected). They give rise to stable wall entrapment so that the motion of microswimmers is mainly along the hard boundary of the obstacle even if, in qualitative difference to steric interactions, the object boundary is convex. This has been seen in experiments and appropriately described by hydrodynamics in Takagi et al. (2014), Schaar, Zöttl, and Stark (2015), and Sipos et al. (2015). Furthermore, a crucial difference emerges between the cases of pushers and pullers discussed in detail in Sec. III.C and in Fig. 11.

\section{B. Active particles in a confined geometry}

In the previous section, we have seen that active particles tend to preferentially spend some time at walls because of the asymmetry between the processes that take them toward and away from boundaries. We now discuss the consequences of this for active particles confined within a finite space such as a pore. We first discuss how non-Boltzmann particle distributions emerge in confined pores, which is in fact a signature of the difference between passive and active particles (Sec. V.B.1). We then introduce the important (and largely open to future investigation) topic of the derivation of an equation of state for active matter systems (Sec. V.B.2). Finally, we deal with the emergence of collective behaviors in confined geometries (Sec. V.B.3).

\section{Non-Boltzmann position distributions for active particles}

We consider a microswimmer confined within a circular pore, as shown in Fig. 24. Figure 24(a) shows four 10-s trajectories of passive Brownian particles $\left(v=0 \mu \mathrm{m} \mathrm{s}^{-1}\right)$ : they uniformly explore the configuration space within the (a)
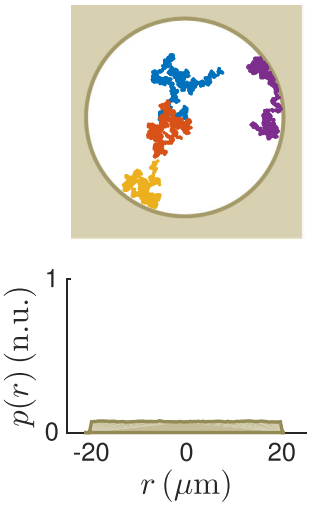

(b)
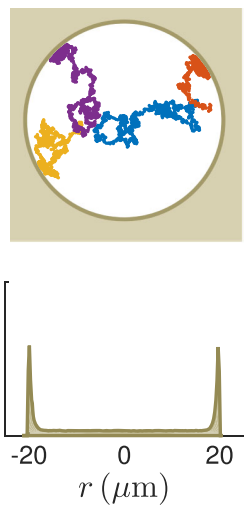

(c)
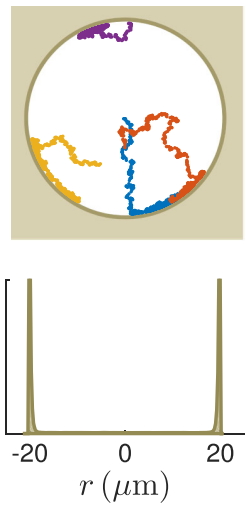

FIG. 24. Non-Boltzmann position distributions for active particles in a pore. (a)-(c) Simulated trajectories (10 s, solid lines) of active Brownian particles (radius $R=1 \mu \mathrm{m}$ ) moving within a circular pore (radius $20 \mu \mathrm{m}$ ) with reflective boundaries at velocity (a) $v=0$, (b) $v=5$, and (c) $v=10 \mu \mathrm{m} \mathrm{s}^{-1}$. The histograms on the bottom show the probability distribution along a diameter of the circular pore: while the probability is uniform across the whole pore in the case of passive Brownian particles, the probability increases toward the walls in the case of active Brownian particles together with the particle velocity and the associated persistence length $L$ [Eq. (6)]. pore. Active particles, shown in Figs. 24(b) $\left(v=5 \mu \mathrm{m} \mathrm{s}^{-1}\right)$ and 24(c) $\left(v=10 \mu \mathrm{ms}^{-1}\right)$, tend to spend more time at the pore boundaries. When a microswimmer encounters a boundary, it keeps on pushing against it and diffusing along the cavity perimeter until the rotational diffusion orients the propulsion of the particle toward the interior of the pore. The chance that the active particle encounters the pore boundary in one of its straight runs increases as its velocity [and thus its persistence length $L$ given by Eq. (6)] increases. These observations can be made more quantitative by using the particle probability distribution. The histograms on the bottom of Figs. 24(a)-24(c) show a section along a diameter of the pore of the probability distribution of finding the particle at the boundaries: it increases together with the particle velocity. This accumulation has been seen in experiments by Bricard et al. (2015).

The fact that active particles tend to accumulate at the boundaries of a pore is a consequence of their out-ofequilibrium nature. For a passive Brownian particle at thermodynamic equilibrium with its environment, the probability distribution $p(x, y)$ is connected to the external potential $U(x, y)$ by the Boltzmann relation $p(x, y) \propto$ $\exp \left\{-U(x, y) / k_{\mathrm{B}} T\right\}$. In the case presented in Fig. 24, there are no external forces acting on the particle and, therefore, $U(x, y)$ and the corresponding Boltzmann distribution are homogeneous, as shown in Fig. 24(a). However, the fact that the distributions in Figs. 24(b) and 24(c) are not homogeneous, despite the homogeneous potential, is a clear deviation from the Boltzmann distribution, i.e., from the behavior of matter at thermodynamic equilibrium.

While the Boltzmann distribution holds for every equilibrium system, a generalized form for the stationary probability distribution characterizing all types of active particles does not exist. However, for some specific classes of active particles analytic solutions can be found, and the traditional Boltzmann equation can also be generalized to some active systems (Schnitzer, 1993; Tailleur and Cates, 2008, 2009; Thüroff, Weber, and Frey, 2013, 2014). The first case is that of noninteracting run-and-tumble particles moving over a onedimensional, conservative force field $f(x)=-d U(x) / d x$. It can be shown that, in a close system with no fluxes, run-andtumble particles will distribute in space with probability (Schnitzer, 1993; Tailleur and Cates, 2008)

$$
p(x)=p\left(x_{0}\right) \frac{D\left(x_{0}\right)}{D(x)} \exp \left[\int_{x_{0}}^{x} \frac{\mu f(s)}{D(s)} d s\right],
$$

where $\mu=\gamma^{-1}$ is the particle's mobility and

$$
D(x)=v^{2} \tau-\mu^{2} f(x)^{2} \tau
$$

is an effective space-dependent diffusion coefficient that is smaller when the magnitude of the external force $f(x)$ is larger. Taking the limit of a vanishing tumble rate $\tau$ while keeping the free-space diffusion $D_{0}=v^{2} \tau$ finite, a Boltzmann-type distribution is recovered with the effective temperature $T_{\text {eff }}=D_{0} / \mu k_{\mathrm{B}}$. In the simple case of a harmonic potential well with stiffness $k$, i.e., $U(x)=k x^{2} / 2$, 
run-and-tumble particles will be confined in a region $|x|<$ $v / \mu k$ with a probability density (Tailleur and Cates, 2009)

$$
p(x)=p(0)\left[1-\left(\frac{\mu k x}{v}\right)^{2}\right]^{\tau / 2 \mu k-1},
$$

which can be very different from the equilibrium Gaussian shape and, in particular, becomes bimodal for $\tau>1 /(2 \mu k)$ with particles accumulating at the edges of the allowed region. These results are exact but valid only for one-dimensional and noninteracting particle systems. For the more generic case of interacting particles in any dimension $d$, a good approximation can be found in the case of colored Gaussian noise. Calling $U(\mathbf{x})$ the potential energy function of an ensemble of $N$ active particles described by the $N d$ coordinate vector $\mathbf{x}$, a flux-free stationary probability distribution can be obtained within the unified colored noise approach (Maggi, Marconi et al., 2015; Marconi and Maggi, 2015) and it reads

$p(\mathbf{x})=\frac{1}{Q} \exp \left[-\frac{U(\mathbf{x})}{D_{0}}-\frac{\tau|\nabla U(\mathbf{x})|^{2}}{2 D_{0}}\right]\|\square+\tau \nabla \nabla U(\mathbf{x})\|$

with $Q$ a normalization factor, $\square$ the $N d$-dimensional identity matrix, and $\|\cdot\|$ representing the absolute value of the determinant. Interestingly, within the same approximation, an analytical expression for the velocity distribution can also be found establishing an explicit link between the mean square velocity and the system's configuration (Marconi et al., 2015). Again, in the limit of vanishing $\tau$, the distribution reduces to the Boltzmann form for an effective temperature $T_{\text {eff }}=D_{0} / \mu k_{\mathrm{B}}$. Contrary to run-and-tumble particles, the onedimensional case of an external harmonic potential always presents a Gaussian shape although with an effective temperature $T_{\text {eff }}=\left(D_{0} / \mu k_{B}\right)(1+2 \mu k \tau)^{-1}$ that depends on the potential curvature. The coexistence of multiple effective temperatures associated with different curvatures in a twodimensional external potential landscape has been observed by Maggi et al. (2014).

Recent studies have generalized the results about the dynamics, diffusivity, and density distributions of active particles in circular confined geometries to geometries of general shape (Sandoval and Dagdug, 2014; Fily, Baskaran, and Hagan, 2015). Ezhilan, Alonso-Matilla, and Saintillan (2015) examined the swim pressure in the regions between two planar walls for run-and-tumble swimmers and found that for large widths the pressure obeys the ideal gas law. At lower widths, however, the pressure deviates from the ideal gas law and decreases at the center since the particles spend more time confined near the walls rather than moving in the bulk.

\section{Active matter forces and equation of state}

When considering active-depletion forces in Sec. IV.C.5, it was shown that active particles can generate (attractive or repulsive) forces between passive plates. This raises the questions of whether such forces depend on the microscopic nature of the interactions of the active particles with the plates and, more generally, of whether an equation of state exists for active particles.
At thermodynamic equilibrium, an ideal gas in a container of volume $V$ can be described by the standard ideal gas equation of state $P=\rho K_{\mathrm{B}} T$, which relates the pressure $P$ to the temperature $T$ and the number density of gas molecules $\rho$. As the temperature increases, the pressure experienced by the container walls increases, independent of the nature or shape of the walls. To address the question of what happens to such a relation when the thermal gas particles are replaced by selfpropelled active particles, Mallory et al. (2014) performed numerical simulations to extract an equation of state for selfpropelled repulsively interacting disks in two- and threedimensional systems and found a nonmonotonic dependence of the pressure on the temperature. By generalizing to active dynamics the definition of pressure as the trace of the microscopic stress tensor, Takatori, Yan, and Brady (2014) and Yang, Manning, and Marchetti (2014) found that mechanical pressure in active systems can be decomposed as the sum of the usual stress component arising from interparticle interactions and a swim pressure component analogous to kinetic pressure in equilibrium systems. In contrast to equilibrium kinetic pressure, however, where particles speed is solely controlled by temperature, swim pressure depends on the actual average speed of the particles that is indirectly controlled by density and persistence length (Solon, Stenhammar et al., 2015). In the special case where particles are not subject to any external torque, either from other particles or from container walls, an equation of state can be derived (Solon, Stenhammar et al., 2015) connecting pressure to bulk properties that is not sensitive to the details of the particles' interaction with the wall. It can also be shown that such an equation of state does not exist in the general case where particles are not torque free (Solon, Stenhammar et al., 2015).

Experimentally, equations of state in colloidal systems can be extracted from sedimentation profiles. Palacci et al. (2010) performed experiments with dense sedimenting suspensions of $\mathrm{H}_{2} \mathrm{O}_{2}$-activated Janus particles, which show a strong change in the sedimentation profile compared to passive systems: after $\mathrm{H}_{2} \mathrm{O}_{2}$ is added to the solution, the solid phase remains but the gas phase spreads to much higher heights. Ginot et al. (2015) performed sedimentation experiments and simulations for active Janus particles and found that activity strongly alters the standard thermodynamic equation of state; however, it was possible to model these alterations using ideas developed through equilibrium concepts. In the dilute case, there is an activity-dependent effective temperature, while at higher densities, an increase in the activity can be modeled as an effective increased adhesion between equilibrium particles.

\section{Collective behaviors in confined geometries}

For microscopic matter that is not at thermodynamic equilibrium, collective behaviors can also emerge in confined geometries due to complex interplay with the surrounding fluid. This can lead to the formation of complex patterns, such as vortices (Hernandez-Ortiz, Stoltz, and Graham, 2005; Yang, Manning, and Marchetti, 2014). These behaviors have been experimentally observed for both swimming bacteria (Wioland et al., 2013; Lushi, Wioland, and Goldstein, 2014) and colloidal rollers (Bricard et al., 2015). Similar behaviors 
have also been reported for vibrated polar granular disks and rods (Kudrolli et al., 2008; Deseigne et al., 2012).

\section{Interaction with obstacles}

The interaction of active particles with obstacles within complex environments lends itself to be exploited in a wealth of potential applications. In general, these may take advantage of the fact that the motion of active particles is nonthermal in order to use the features of the environment to perform complex tasks such as the separation, trapping, or sorting of active particles on the basis of their swimming properties. In this section we explore these possibilities; in particular, we consider how active particles can be gathered by wedges (Sec. V.C.1), directed by ratcheted walls (Sec. V.C.2), funneled along patterned channels (Sec. V.C.3), sorted in periodic arrays of obstacles (Sec. V.C.4), and trapped within random environments (Sec. V.C.5).

\section{Capture and concentration of active particles}

In many applications, it is important to catch groups of autonomously navigating microbes and man-made microswimmers in a controlled way. This can be achieved, for example, by using some obstacles in the shape of wedges. The condition for trapping is a pretty sharp cusp on the length scale of the swimmer extension. For example, Kaiser, Wensink, and Löwen (2012) considered active self-propelled rods interacting with stationary wedges as a function of the wedge angle $\alpha$, finding three regimes of behavior, whose phase diagram is shown in Fig. 25(a): for small angles, a partial trapping of the active particles occurs [Fig. 25(b) for $\alpha=40^{\circ}$ ]; for intermediate angles, complete trapping of the particles occurs [Fig. 25(c) for $\alpha=116^{\circ}$ ]; and, for large angles, there is no longer any trapping of the particles [Fig. 25(d) for $\alpha=140^{\circ}$ ]. We remark that the trapping by wedges is distinct from carrier motion discussed in Fig. 18 because it is triggered and selfamplified by the strong aligning excluded-volume forces between the rods, while carrier motion already occurs for an ideal gas of swimmers. In a subsequent work, Kaiser et al. (2013) found enhanced trapping when the wedge is moved in certain orientations (see Sec. IV.C.4). This trapping behavior can be enhanced employing a system of multilayered asymmetric barriers (Y.-F. Chen et al., 2015). The self-trapping behavior of active rods was then confirmed in experiments on artificial rodlike swimmers (Restrepo-Pérez et al., 2014) and sperm cells (Guidobaldi et al., 2014).

Galajda et al. (2007) placed swimming bacteria in a confined area containing an array of funnel shapes [Figs. 26(a) and 26(b)] and observed that the bacteria concentrated in the portion of the container toward which the funnel apertures pointed, indicating that the bacteria were undergoing a ratchet motion [Figs. 26(c)-26(e)]. Wan et al. (2008) performed simulations of a simplified model of the system in which the bacteria are treated as run-and-tumble particles that move along any wall they encounter. For small run lengths, the system behaves thermally and no ratchet effect occurs; however, for longer run lengths a ratchet effect emerges and, just as in the experimental case, there is a buildup of simulated bacteria on one side of the container. The simulations also showed a buildup of active particle density in the tips of the funnel barriers. Tailleur and Cates (2009) studied run-and-tumble particles interacting with walls and observed a buildup of particle density along the walls as well as a ratchet effect in the presence of funnel-shaped walls. They found that changing the interaction of the particles with the wall, e.g., by introducing a reflection of the particles from the wall, can destroy the ratchet effect. Galajda et al. (2008) constructed a macroscale version of the ratchet system and observed the same behavior as in the bacterial version, indicating that detailed hydrodynamic interactions with the walls are not solely responsible for the ratchet effect.

\section{Ratchet effects and directed motion}

Thermal Brownian particles moving over an asymmetric substrate do not spontaneously drift in one direction (if the spatial average of the force is zero). If, however, an external periodic drive is added, or if the substrate is flashed on and off to create nonequilibrium conditions, a net continuous drift of particles known as a ratchet effect can arise (Reimann, 2002).

Numerous studies of ratchet effects have been performed for a variety of active matter systems in the presence of (a)

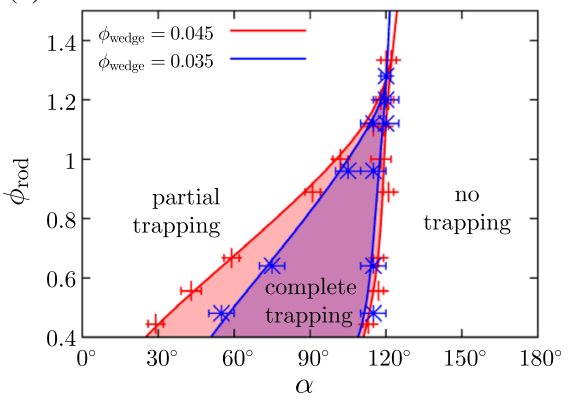

(b)

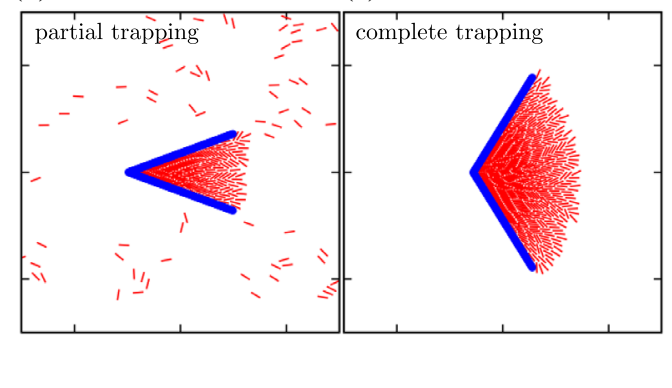

(d)

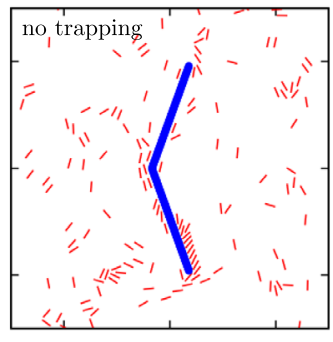

FIG. 25. Capturing active particles with a wedge. (a) Phase diagram marking three different collective trapping states of self-propelled rods at a wedge upon variation of the reduced rod packing fraction $\left(\phi_{\text {rod }}\right)$ : no trapping at large apex angle $\alpha$, complete trapping at medium $\alpha$, and partial trapping at small $\alpha$. Phase boundaries are shown for two different values of the area fraction occupied by the wedge $\left(\phi_{\text {wedge }}\right)$. The region of complete trapping is bounded by a triple point at larger rod concentration beyond which a smooth transition from no trapping to partial trapping occurs. (b)-(d) Snapshots depicting the examples of the three stationary states. From Kaiser, Wensink, and Löwen, 2012. 
(a)

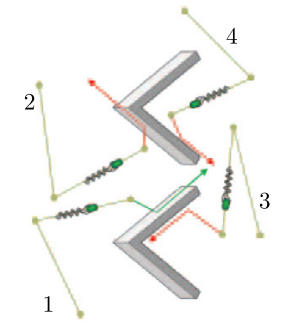

(b)

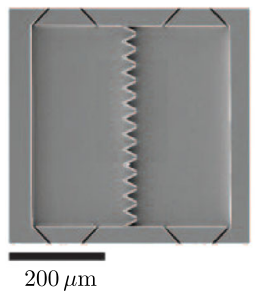

(c)

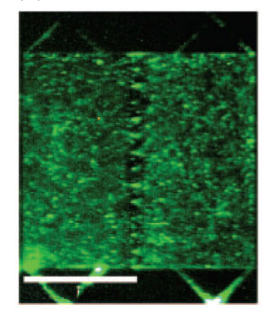

(d)

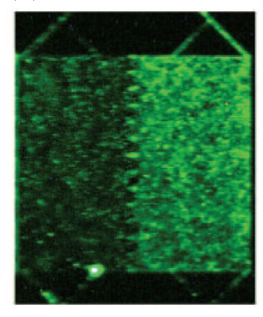

(e)

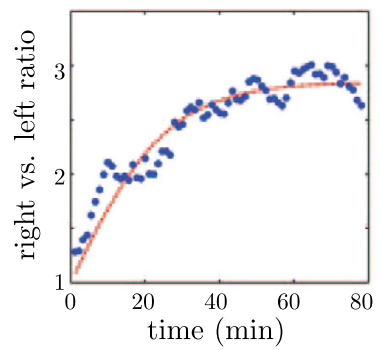

FIG. 26. Concentration of active particles. (a) Schematic drawing of the interaction of bacteria with a funnel opening: on the left side, bacteria may (trace 1) or may not (trace 2) get through the gap, depending on the angle of attack. On the right, almost all bacteria colliding with the wall are diverted away from the gap (traces 3 and 4). (b) Scanning electron micrograph of the device. (c) Uniform distribution of bacteria in a structure with a funnel wall after injection and (d) steady-state distribution after 80 min. (e) Ratio of densities in the right and left compartments vs time: the circles are experimental data, and the dashed line is a fit to the relative theory. From Galajda et al., 2007.

asymmetric barriers, including swimming eukaryotes (Kantsler et al., 2013), swimming sperm cells (Guidobaldi et al., 2014), active particles moving in corrugated channels (Ai et al., 2013; Pototsky, Hahn, and Stark, 2013; Koumakis, Maggi, and Di Leonardo, 2014; Yariv and Schnitzer, 2014), active polymers (Wan and Jho, 2013), active ellipsoids (Ai and Wu, 2014), crawling cells (Mahmud, Campbell, and Grzybowski, 2009), and systems containing a variety of asymmetric objects with varied swimming strategies (Berdakin et al., 2013; Ghosh et al., 2013; Ai, He, and Zhong, 2014; Potiguar, Farias, and Ferreira, 2014).

Variations of the active ratchet effect include active drift ratchets, in which an external continuous drive is applied to the active particles as they pass through an array of asymmetric obstacles (Volpe et al., 2011; Reichhardt and OlsonReichhardt, 2013a). There are also chiral ratchet effects that arise for active particles that have an intrinsic swimming asymmetry oriented in either the clockwise or counterclockwise direction (Mijalkov and Volpe, 2013; Reichhardt and Olson-Reichhardt, 2013b). When such particles are placed in an asymmetric substrate, particles with different chiralities move in different directions, permitting the chiral species to be separated. The next step along this research line is to (experimentally) realize substrate geometries to sort chiral active particles on the nanoscale, which could have numerous applications in biological and medical sciences, as seen in more detail in Sec. V.D.

It is also possible to obtain a reversed ratchet effect in which the particles move in the forward ratchet direction for one set of parameters but in the reverse direction for a different set of parameters. Drocco, Olson-Reichhardt, and Reichhardt (2012) demonstrated a reversible ratchet effect for active flocking particles moving through an array of funnel barriers. They simulated a variant of the Vicsek flocking model in which the particles have an additional short-range steric repulsion in order to give the flocks a finite size. In the dilute or high-noise limit, the particles are disordered and ratchet in the forward or normal direction through the funnels. However, for densities or noise levels in the collective or flocking regime, flocks become effectively jammed when they attempt to pass through the funnel due to the incompressibility of the flock, which behaves as a rigid solid. In contrast, a flock approaching the funnel array from the hard flow direction can split in two and lose a portion of its members to the other side of the array, resulting in a net reversed ratchet motion of the particles. Experimental studies of crawling cells also showed that it is possible for one species of cells to ratchet in one direction through an asymmetric environment while another species of cells ratchets in the opposite direction (Mahmud, Campbell, and Grzybowski, 2009).

Koumakis et al. (2013) demonstrated that a ratchet effect can also be induced on passive colloidal particles that jump over asymmetric barriers when pushed by swimming bacteria. As shown in Fig. 27, the escape rate over an asymmetric barrier was found to be higher when the same barrier is approached from the small slope side. By surrounding a target region with asymmetric walls, colloidal particles can accumulate over the target when the high slope sides of the barriers point inside, or clear the target region when higher slopes point outside. This effect can be interpreted in terms of a nonuniform effective temperature (Koumakis, Maggi, and Di Leonardo, 2014). Ghosh et al. (2013) performed numerical simulations of active Janus particles in asymmetric funnel geometries containing additional passive particles and found that even a small number of active particles can induce a directed motion of the passive particles.

The works described in this section indicate that active ratchet effects can be employed in techniques aiming at the separation, mixing, or directed flow of passive particles.

\section{Motion rectification in a microchannel}

The motion of active particles can be rectified by a patterned microchannel [a recent topical review was given by Ao et al. (2014)]. The inset of Fig. 28(c) shows an example of such a microchannel decorated with a series of asymmetric dents on both its walls. Differently from a group of passive Brownian particles released at time $t=0 \mathrm{~s}$ from position $x=0 \mu \mathrm{m}$ which diffuse symmetrically around the initial position [filled histograms in Figs. 28(a)-28(c)], a group of active Brownian particles is funneled by the channel in such a way that an average directed motion is imposed on the particles, as can be seen in the open histograms in Figs. 28(b) and 28(c). The rectification is the more 

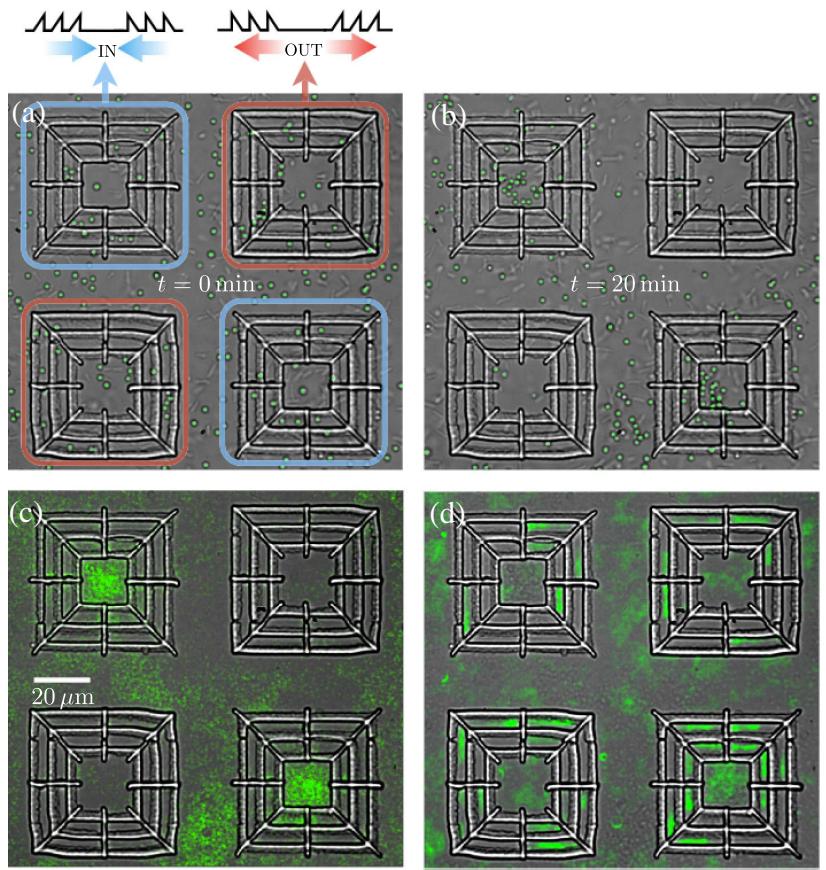

FIG. 27. Observation of particle concentration and depletion by bacteria. Snapshots of particles and bacteria (a) at $t=0$ (the initial state), where particles are randomly distributed, and (b) at $t=20 \mathrm{~min}$, where particle distributions have been strongly affected by bacterial transport over asymmetric barriers. The colloidal particles that are not stuck on the surface are highlighted. Particle distributions averaged over a steady state (c) for particles in the bacterial bath between $t_{1}=15$ and $t_{2}=20 \mathrm{~min}$ $(\Delta t=5 \mathrm{~min})$ and (d) for particles in an experiment without bacteria, undergoing simple Brownian motion for $\Delta t=10 \mathrm{~min}$. In the absence of bacteria, the colloidal particles remain trapped within the structures' compartments. From Koumakis et al., 2013.

pronounced the higher the velocity of the active particle. This and similar effects have been proposed to sort microswimmers on the basis of their velocity or size (Hulme et al., 2008; Mijalkov and Volpe, 2013), to trap microswimmers in moving edges (Kaiser et al., 2013), to deliver microscopic cargoes to a given location (Koumakis et al., 2013), to convey a strategic advantage in trespassing channels against external biases (Locatelli et al., 2015), and even to control pedestrian flows (Oliveira et al., 2016).

\section{Extended landscapes of obstacles}

The motion and properties of active particles can also be influenced by the presence of extended potential landscapes or by the presence of obstacles in the environment.

Reichhardt and Olson-Reichhardt (2014b) performed a numerical study of interacting run-and-tumble disks of radius $R$ moving under a drift force $F_{\mathrm{d}}$ through an assembly of immobile disks that serve as a disordered landscape of obstacles. This is similar to a system of ordinary chargestabilized colloidal particles driven with an electric field over an obstacle array, where the transport can be characterized by the average velocity $\left\langle v_{\mathrm{d}}\right\rangle$ of the particles in the direction of the applied drift force. For the passive particles, when no (a)

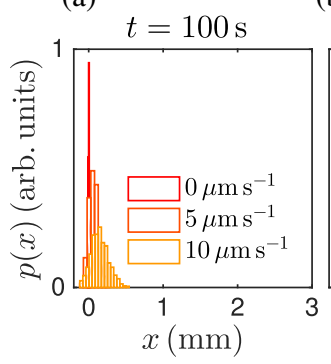

(b)

$t=500 \mathrm{~s}$

(c)

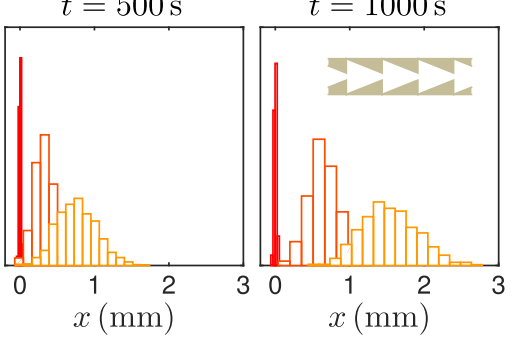

FIG. 28. Rectification of active Brownian motion in an asymmetric ratchetlike microchannel. The distribution of passive [dark gray (red) histograms] and active [light gray (yellow) histograms] Brownian particles (radius $R=1 \mu \mathrm{m}$ ) released at time $t=0 \mathrm{~s}$ from position $x=0 \mathrm{~mm}$ are plotted at times (a) $t=100$, (b) $t=500$, and (c) $t=1000 \mathrm{~s}$. The higher the active particle velocity, the farther the active particles travel along the channel. Every histogram is calculated using 1000 particle trajectories. A segment of the channel, whose dent is $10 \mu \mathrm{m}$ long, is represented by the gray structure in the inset of (c).

obstacles are present, $\left\langle v_{\mathrm{d}}\right\rangle$ increases linearly with the magnitude $F_{\mathrm{d}}$ of the drift force, i.e., $\left\langle v_{\mathrm{d}}\right\rangle \propto F_{\mathrm{d}}$, indicating Ohmic behavior where the damping arises from the Stokes flow. When obstacles (or pinning) are present, there can be a finite critical external driving (or depinning threshold) force $F_{\mathrm{c}}$ required to set the particles in motion, so that $\left\langle v_{\mathrm{d}}\right\rangle=0$ when $F_{\mathrm{d}}<F_{\mathrm{c}}$. Once the particles are moving, $\left\langle v_{\mathrm{d}}\right\rangle$ may increase linearly with $F_{\mathrm{d}}$ or it may follow a power law $\left\langle v_{\mathrm{d}}\right\rangle \propto F_{\mathrm{d}}^{\alpha}$. When thermal fluctuations are also present, the true critical depinning threshold is lost due to particle creep, and the velocity follows the form $\left\langle V_{\mathrm{d}}\right\rangle \propto v \exp \left(-U_{\mathrm{p}} / k_{\mathrm{B}} T\right)$, where $v$ is the velocity of the particle in the obstacle-free limit and $U_{\mathrm{p}}$ is the effective trapping potential created by the disorder. Here the velocity decreases with increasing $U_{\mathrm{p}}$ or decreasing $T$, so that increasing the magnitude of the thermal fluctuations always produces a larger drift velocity. Similarly, increasing $T$ always leads to a larger diffusion of particles in the presence of random disorder. If the fluctuations are active rather than thermal, the velocity can behave very differently. Figure 29(a) shows an image of a system where active particles are moving through an assembly of obstacles. Some plots of $\left\langle v_{\mathrm{d}}\right\rangle$ versus run length $R_{1}$ for the same system are shown in Fig. 29(b). ${ }^{7}$ At $R_{1}=0$, most of the particles become trapped and $\left\langle v_{\mathrm{d}}\right\rangle$ is low. As $R_{1}$ increases, the fluctuations assume a thermal character and local clogs in the assembly break apart, allowing particles to flow in the drift direction and increasing $\left\langle v_{\mathrm{d}}\right\rangle$. When $R_{1}$ is further increased, however, the drift velocity begins to decrease, as shown in Fig. 29(b) by the fact that $\left\langle v_{\mathrm{d}}\right\rangle$ reaches its maximum value near $R_{1}=1$. The drop in velocity is correlated with an onset of clustering (or phase-separation effect) that occurs in active disk systems for long run lengths or high activity. In the regime of low but finite activity, the particles form a liquid state, so an individual particle is at most

${ }^{7}$ Because these plots show a crossing due to the nonmonotonicity of the drift velocity for varied run lengths, they are more informative than plotting the drift velocity versus force since those curves are generally linear. 

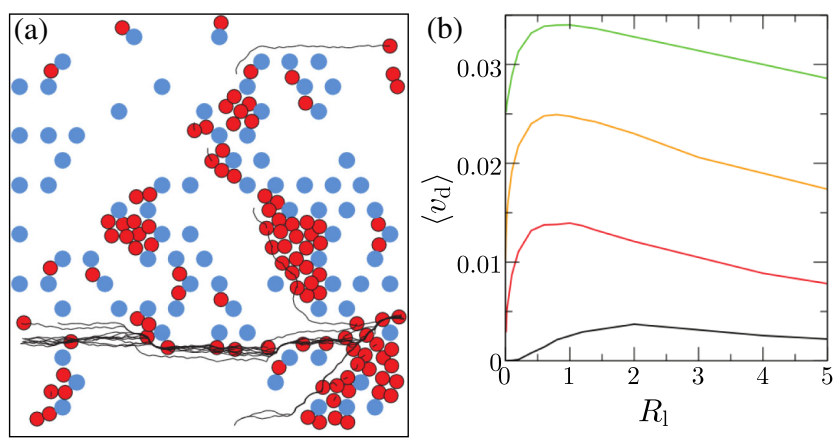

FIG. 29. Effect of a disordered landscape on the driving velocity of active particles. (a) Positions of pinned disks [light gray (blue) circles], active disks [dark gray (red) circles], and disk trajectories over a period of time (lines) in a small section of a sample with obstacle area fraction $\phi_{\mathrm{p}}=0.0235$, total area fraction $\phi=0.188$, and run length $R_{1}=0.004$. (b) The average drift velocity $\left\langle v_{\mathrm{d}}\right\rangle$ as a function of $R_{1}$ passes through a maximum for all the curves; $\phi_{\mathrm{p}}=0.055,0.094,0.1413$, and 0.188 , from top to bottom. From Reichhardt and Olson-Reichhardt, 2014b.

temporarily trapped behind an obstacle before diffusing around it. When the activity level increases and the system enters the phase-separation regime, the formation of clusters of particles makes it possible for an individual obstacle to trap an entire cluster. Further work can be envisaged on this topic; in particular, it would be interesting to determine how the depinning transition is rounded by the particle's activity as well as to study the system's behavior at finite but large run times.

It is possible to create a random energy landscape for active particles using speckle patterns which can be generated by an optical field (Volpe et al., 2014; Volpe, Volpe, and Gigan, 2014; Pesce et al., 2015). Paoluzzi, Di Leonardo, and Angelani (2014) performed molecular dynamics simulations of swimming bacteria interacting with a speckle pattern. The swimmers are modeled as dumbbell particles and interact through short-range repulsive interactions. A crossover is observed from nontrapping to trapping of the particles as the speckle intensity is increased. Pinçe et al. (2016) demonstrated that the presence of spatial disorder can alter the long-term dynamics in a colloidal active matter system, making it switch between gathering and dispersal of individuals: at equilibrium, colloidal particles always gather at the bottom of any attractive potential. However, under nonequilibrium driving forces in a bacterial bath, the colloids disperse if disorder is added to the potential; the depth of the local roughness in the environment regulates the transition between gathering and dispersal of individuals in the active matter system, thus inspiring novel routes for controlling emerging behaviors far from equilibrium.

Reichhardt and Olson-Reichhardt (2014a) considered an active matter system composed of run-and-tumble repulsive disks in the limit of an infinite run time. Using a periodic system, they found that for a sufficiently large density of active particles, most of the particles become trapped in a single large clump and all dynamical fluctuations in the system vanish. The large clump can still be translating, but all trajectories of all particles are periodic. The transient time required to reach such a state diverges as a power law at the critical particle density, suggesting that this is an example of a nonequilibrium phase transition from a strongly fluctuating state to a dynamically frozen state. Such transitions are known as absorbing phase transitions, and they have been studied experimentally and numerically for actin motion, where the system can transition from a fluctuating state to an ordered spiral state containing almost no fluctuations (Reichhardt and Olson-Reichhardt, 2011; Schaller et al., 2011). Reichhardt and Olson-Reichhardt (2014a) added obstacles to the system of infinitely running particles as illustrated in Fig. 30. These obstacles act as nucleation sites for clusters, as shown in Fig. 30(a). Inside a cluster, particles are swimming against each other and are held in the cluster configuration by steric repulsion. In Fig. 30(d) the particles are colored according to the average $y$ component of the active force, making it clear that the upper half of the cluster is moving downward in opposition to the lower half of the cluster which is moving upward, resulting in a completely pinned cluster. When obstacles are present, clusters can form even for very low
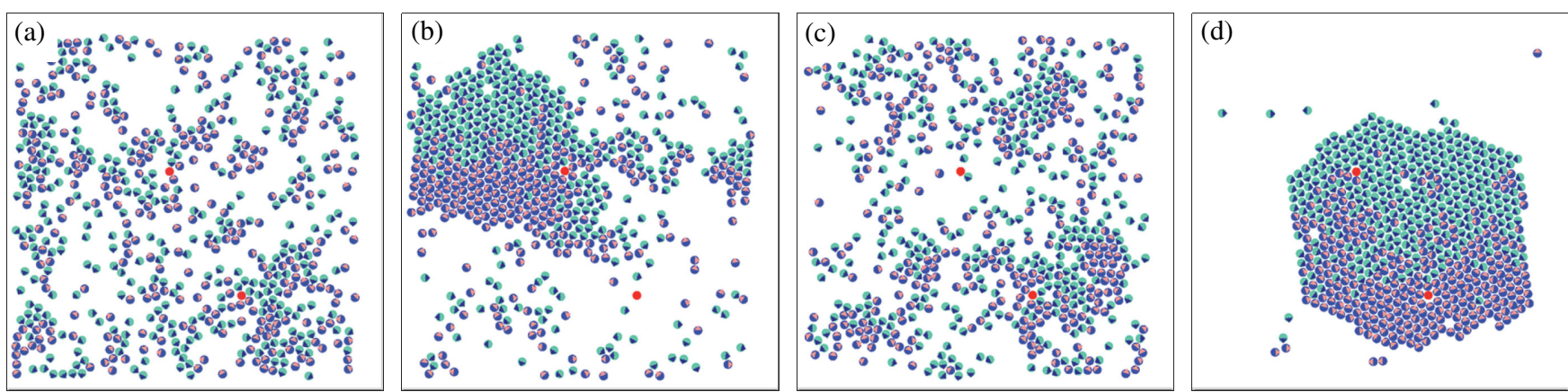

FIG. 30. Cluster formation induced by obstacles. Snapshots of the positions of active disk and obstacles in a system with area fraction $\phi=0.363$ and number of obstacles $N_{\mathrm{p}}=2$. The arrows indicate the direction of the motor force for each disk. Dark particles with light arrows have a net motion in the positive $y$ (vertical) direction; light particles with dark arrows have a net motion in the negative $y$ direction. Red disks are immobile obstacles. (a) The initial fluctuating state contains small transient clusters. (b) After some time, transient clusters are nucleated by the obstacles. (c) At a still later time, the transient cluster shown in (b) has broken apart. (d) Finally, the dynamically frozen steady state appears where a faceted crystal forms around the obstacles. From Reichhardt and Olson-Reichhardt, 2014a. 
active particle densities. Since the active disks are monodisperse in size, triangular ordering appears inside the cluster, which can lead to faceted crystals, as shown in Fig. 30(d). When the obstacle density is higher, the system reaches the frozen state much more rapidly and the cluster becomes increasingly disordered, as shown in Figs. 30(b) and $30(\mathrm{c})$. In the absence of obstacles, the dynamically frozen state forms for active disk densities $\phi>0.5$; however, as the obstacle density $\phi_{\mathrm{p}}$ increases, the density at which the dynamically frozen state appears decreases. Kumar, Ramaswamy, and Sood (2011) considered the large-deviation function of a single polar active particle in a crowded structured environment and Kumar et al. (2014) considered the flocking of active polar objects in a crowded nonmotile background, where the crowdedness is crucial to producing the ordering.

In addition to swimming particles, there have been studies of flocking particles interacting with obstacle arrays. Chepizhko, Altmann, and Peruani (2013) considered a variant of the Vicsek model for self-propelled particles interacting with a heterogeneous environment of obstacles. When a particle interacts with an obstacle, it turns its velocity vector away from the obstacle once it comes within a certain distance from it. There is a stochastic noise term added to the particle alignment direction which affects not only its flocking behavior but also its interaction with the obstacles. In the absence of obstacles, the system reduces to the Vicsek model, in which increasing the magnitude of the noise term results in a transition from a coherent state in which all particles move in the same direction to a disordered state in which the direction of motion is randomized. When obstacles are present, Chepizhko, Altmann, and Peruani (2013) found that the system is disordered when the noise term is small. However, as the noise term increases in magnitude, the system transitions into a state with quasi-long-range order, while for the highest values of noise the system is disordered again. This result indicates that the addition of some noise can induce ordering or coherent motion in an active matter system and can be regarded as an example of producing order through disorder. This effect arises since the noise tends to wash out the effect of the quenched disorder array and to make the particle density more homogeneous; however, when the noise is large enough, the system becomes disordered again.
Another system that has been explored is chiral active particles in random landscapes. Nourhani, Crespi, and Lammert (2015) considered chiral self-propelling particles moving on a periodic potential where they found that particles of different chirality can be separated. They also observed a variety of distinct subclasses of dynamic states in which the particles form orbits that either have a translational propagation or are localized. Another feature of this system is that it is possible to steer particles to arbitrary locations by changing the strength of the substrate. Schirmacher et al. (2015) considered a model of noninteracting particles undergoing circular motion in a random landscape to model electrons in a magnetic field moving through random disorder; however, the same model can be used to represent circularly swimming particles. When the swimming radius decreases, there is a transition from a delocalized to a localized state.

\section{Subdiffusion and trapping of microswimmers}

Various emergent phenomena have been observed when active particles interact with a disordered environment such as subdiffusion and trapping.

Chepizhko and Peruani (2013) considered the diffusion and trapping of active particles in the presence of obstacle arrays using the same flocking model described by Chepizhko, Altmann, and Peruani (2013) (see Sec. IV.C.4). As shown in Fig. 31(a), they studied the system as a function of the turning rate of the particles. They found that the motion is diffusive when the turning rate is small; however, the diffusion constant depends nonmonotonically on the particle density. For high obstacle densities and large turning rates, the obstacles induce particle trapping, producing subdiffusive motion. By exploiting this effect, it could be possible to perform a filtering of active particles using an obstacle array, where a portion of the particles with specific properties would become trapped in the array while other types of particles could diffuse freely across it.

Quint and Gopinathan (2013) studied a swarming model in the presence of static disorder. They observed that even a small amount of disorder can suppress the ordering of the swarm when only aligning interactions are included. However, when additional repulsive forces between particles are added, they found a transition from a collectively moving state to a gaslike state at a finite amount of disorder. This (a)

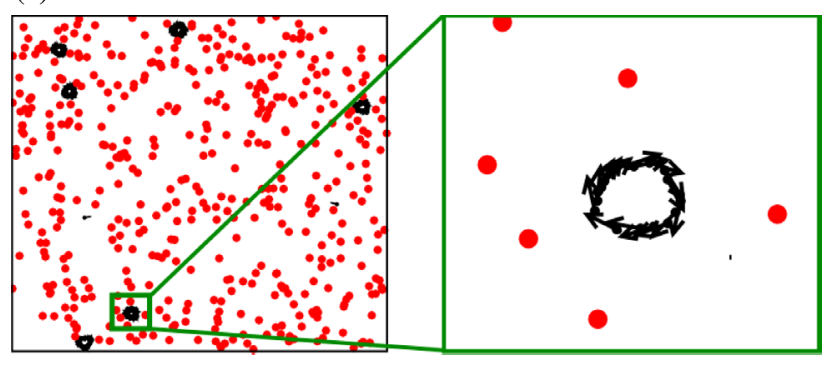

(b)

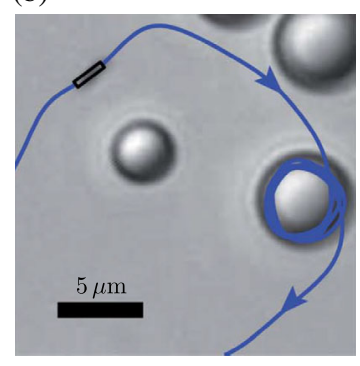

FIG. 31. Trapping of active particles. (a) In a system where the particle orientation is affected by the presence of obstacles, spontaneous particle trapping occurs for large values of the turning speed and of the obstacle density. Obstacles are indicated by gray (red) dots while black arrows correspond to active particles. From Chepizhko and Peruani, 2013. (b) Sample trajectory of a self-propelled rod orbiting around a passive sphere. The sphere has diameter $6 \mu \mathrm{m}$, the rod length is $2 \mu \mathrm{m}$, and its speed is on the order of $20 \mu \mathrm{m} \mathrm{s}{ }^{-1}$. From Takagi et al., 2014. 
transition resembles a percolation transition; however, the transition occurs at densities well below those at which standard percolation would be expected to appear. Chepizhko and Peruani (2015) also numerically considered swarming particles in heterogeneous media.

Studies of active particles interacting with obstacles are often performed in the limit where hydrodynamic effects can be neglected. Takagi et al. (2014) experimentally studied chemically propelled microrods interacting with large spheres and found that the rods become trapped in circles around the spheres as illustrated in Fig. 31(b). The behavior of the trapped rods can be explained using a model that includes the hydrodynamic interactions between the rod and the surface of the sphere. Spagnolie et al. (2015) performed a numerical and theoretical analysis of the capture of microswimmers interacting with obstacles, showing that the time during which the swimmers are trapped by the obstacles before escaping has a distribution with a long tail. Similar hydrodynamic trapping effects were observed in bacteria swimming near large convex obstacles (Sipos et al., 2015).

\section{Sorting of microswimmers}

Sorting microswimmers based on their swimming properties (e.g., velocity, angular velocity, and chirality) is of utmost importance for various branches of science and engineering. Genetically engineered bacteria can be sorted based on phenotypic variations of their motion (Berg, 2004). Velocity-based spermatozoa selection can be employed to enhance the success probability in artificial fertilization techniques (Guzick et al., 2001). Considering the intrinsic variability of microfabrication techniques, the efficiency of artificial microswimmers for a specific task, e.g., drugdelivery or bioremediation, can be increased by selecting only those with the most appropriate swimming properties. In this section we explore how complex environments can be used to sort microswimmers based on their swimming style; in particular, we consider static patterns (Sec. V.D.1) and chiral sorting (Sec. V.D.2).

\section{Static patterns}

Two-dimensional periodic patterns can be used to select active particles based on their swimming style. Volpe et al. (2011) investigated the behavior of self-propelled particles in the presence of a two-dimensional periodic pattern where straight unlimited swims are possible along only certain directions. The structure was made of a series of ellipsoidal pillars arranged in a triangular lattice [lattice constant $L_{\mathrm{c}}=35 \mu \mathrm{m}$, Fig. 32(a)]. Within such structure, long swimming runs are possible along only two main directions: at $\pm 60^{\circ}$ and $\pm 90^{\circ}$ with respect to the $y$ axis. Otherwise the motion is strongly hindered due to collisions with the obstacles. The addition of a drift force $F_{\mathrm{d}}$ along the $y$ direction leads to strong differences in the particle trajectories depending on their swimming length.

The typical trajectory of a Brownian particle is shown in Fig. 32(a). When compared to the effect of the drift force, the effect of the diffusion is rather weak so that the particle meanders almost deterministically through the structure in the direction of $F_{\mathrm{d}}$. For increasing swimming lengths $L$, however, significant changes in the trajectories are observed. These become particularly pronounced for $L>L_{\mathrm{c}}$, where the particles perform swimming runs of increasing length along the diagonal channels [Figs. 32(c) and 32(d)]. For $L=83 \mu \mathrm{m}$ the propulsion becomes so strong that the particles partially move perpendicular to the drift force [Fig. 32(e)]; occasionally even motion against the drift force can be observed.

The direction of the particle motion through the structure is characterized by the direction (with respect to the $y$ axis) of the line connecting points of the trajectory separated by a distance of $100 \mu \mathrm{m}$. The probability distributions of these angles are shown by the light gray (red) polar histograms in Figs. 32(f)-32(j). One observes that with increasing $L$ the propagation of particles along the direction of the applied drift becomes less likely, while trajectories along $\pm 60^{\circ}$, i.e., along the directions that permit long swimming events, become more frequent. Differently from the deflection of Brownian particles in a periodic potential (MacDonald, Spalding, and (a)

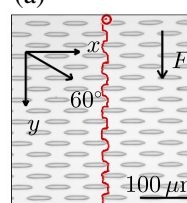

(f)

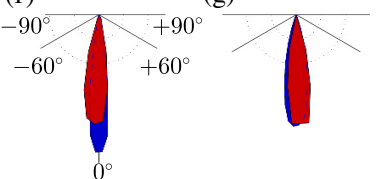

(c)

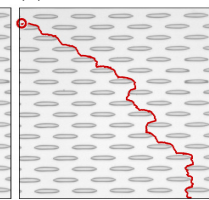

(h)

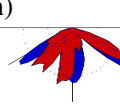

(d)

$(\mathrm{e})$

(c)

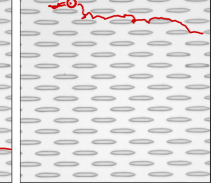

(i)

(j)

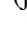

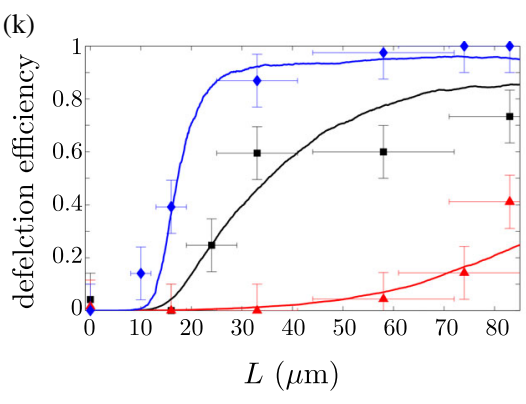

FIG. 32. Sorting of microswimmers in a periodic environment. (a)-(e) Typical trajectories of self-propelled particles moving through a triangular lattice (lattice constant $L_{\mathrm{c}}=35 \mu \mathrm{m}$ ) of elliptical obstacles when a drift force $F_{\mathrm{d}}=0.12 \mathrm{pN}$ is applied along the $y$ direction. The characteristic swimming length is (a) $L=0$ (Brownian particle, no propulsion), (b) 16, (c) 24, (d) 33, and (e) $83 \mu \mathrm{m}$. (f)(j) Corresponding histograms of the experimentally measured [light gray (red)] and simulated [dark gray (blue)] directions of the particle trajectories as defined by two points in the trajectory separated by $100 \mu \mathrm{m}$. The experimental histograms were obtained considering more than 100 trajectories in each case. (k) Measured probability that particles are deflected by more than $30^{\circ}$ after a traveling length of $100 \mu \mathrm{m}$ as a function of $L$ for various imposed drift forces $F_{\mathrm{d}}=0.06 \pm 0.02$ (diamonds), $0.12 \pm 0.05$ (squares), and $0.28 \pm 0.12 \mathrm{pN}$ (triangles). The solid lines are the results of numerical calculations. From Volpe et al., 2011. 
Dholakia, 2003; Huang et al., 2004; Reichhardt and OlsonReichhardt, 2004), this mechanism relies on the dynamical properties of the microswimmers. These results were also compared with numerical simulations [dark gray (blue) polar histograms in Figs. 32(f)-32(j)], which show good agreement with the experimental data.

With the additional possibility of varying the drift force, these observations can be exploited to spatially separate selfpropelled particles with small differences in their individual swimming behavior [Fig. 32(k)]. The sorting mechanism discussed here can be directly applied to other self-propelled objects; in these cases, drift forces can be created, e.g., by electric fields or by a solvent flow through the device.

\section{Chiral particle separation}

Active particles can also be separated on the basis of their chirality (Mijalkov and Volpe, 2013; Reichhardt and OlsonReichhardt, 2013b; Ai, He, and Zhong, 2015; Chen and Ai, 2015; Wu, Chen, and Ai, 2015). This is a particularly interesting option because it may provide a better technique to separate levogyre and dextrogyre chiral molecules by chemically coupling them to chiral propellers, sorting the resulting chiral microswimmers, and finally detaching the propellers, as theoretically suggested by Mijalkov and Volpe (2013). This is interesting because often only one specific chirality is needed by the biochemical and pharmaceutical industries (Ahuja, 2011), and separation can hardly be achieved by mechanical means due to the extremely small Reynolds numbers (Marcos, Powers, and Stocker, 2009). Figure 33 shows a possible approach to sorting active particles based on the sign of their motion chirality in the presence of some chiral patterns in the environment (Mijalkov and Volpe, 2013), such as arrangements of tilted rectangles along circles forming chiral flowers. Two chiral flowers with opposite chiralities are enclosed in a $100-\mu \mathrm{m}$-side box where the particles can move freely. At time $t=0 \mathrm{~s}$ a racemic mixture is placed inside each flower [Fig. 33(a)]. With time passing by, most of the levogyre (dextrogyre) microswimmers escape the right (left) chiral flower, while the ones with the opposite chirality remain trapped. At $t=1000 \mathrm{~s}$ most of the microswimmers are stably trapped, as can be seen in Fig. 33(c).
Other approaches have also been proposed to separate chiral microswimmers, e.g., periodically patterned channels (Mijalkov and Volpe, 2013; Y. Li et al., 2014; Ai, He, and Zhong, 2015; Ao et al., 2015) and rotary obstacles (Chen and Ai, 2015).

\section{TOWARD THE NANOSCALE}

Scaling down microswimmers to the nanoscale and, therefore, implementing effective nanoswimmers is an open technological and scientific challenge (Peplow, 2015). Being successful in this task would be extremely beneficial: it would in fact allow the scaling down of several technologies both increasing their efficiency and decreasing their footprint. The main problem on the way toward the nanoscale is that as the size of an active particle decreases, its motion becomes more random and less directed mainly because of an increase in the particle's rotational diffusion. In fact, by comparing Eqs. (1) and (2), we can see that, while $D_{\mathrm{T}}$ scales according to $R^{-1}, D_{\mathrm{R}}$ scales according to $R^{-3}$, so that when we approach nanometric sizes, $\tau_{\mathrm{R}}$ becomes very short. For instance, for a nanoparticle with hydrodynamic radius $R=50 \mathrm{~nm}, \tau_{\mathrm{R}}=760 \mu \mathrm{s}$. Thus, self-propulsion does not have enough time to alter the way in which a nanoswimmer interacts with its environment; its main effect is, instead, to enhance the particle's diffusion, generating what has been refereed to as hot Brownian motion (Rings et al., 2010).

Nevertheless, not everything is lost. In fact, different solutions can be brought into play to outbalance the increased rotational diffusion at nanometric swimmers' sizes, e.g., by exploiting the following:

Taxis: Taxis is the movement of a system in response to an external stimulus such as light or the presence of a chemical plume. For example, nanoswimmers can be designed to respond to the gradient in an external (e.g., chemical, optical, acoustic) field that powers their propulsion mechanism so that a long-term drift appears in their motion as a response to the gradient. The gradient can even counteract the rotational diffusion by inducing alignment of the swimmers. Because of the increased rotational motion at the nanoscale, however, (a)

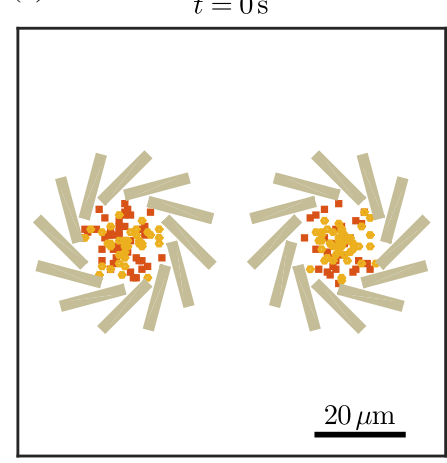

(b)

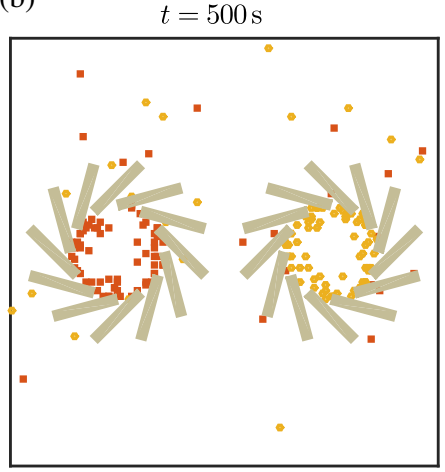

(c)

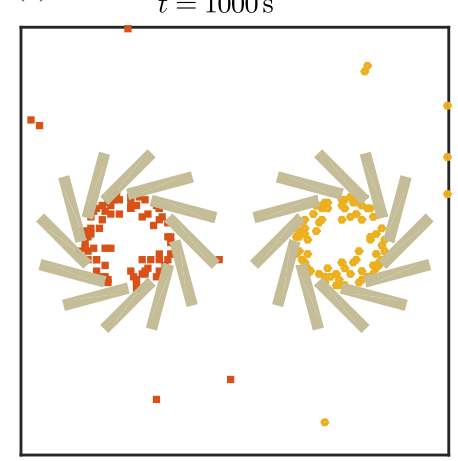

FIG. 33. Sorting of chiral microswimmers $\left(R=1 \mu \mathrm{m}, v=31 \mu \mathrm{m} \mathrm{s}^{-1}\right.$, and $\omega= \pm 3.14 \mathrm{rad} \mathrm{s}^{-1}$ ) with chiral flowers (rectangles). (a) At $t=0 \mathrm{~s}$ a racemic mixture of active particles is released inside two chiral flowers with opposite chiralities. (b), (c) As time progresses, the levogyre [dark gray (red) squares] active particles are trapped in the left chiral flower, while the dextrogyre ones [light gray (yellow) circles] are trapped in the right chiral flower. 
there exists a size limit of $\approx 600 \mathrm{~nm}$ below which tactic strategies (e.g., swim-and-tumble motion) are no longer efficient (Dusenbery, 1997).

Environmental properties: Nanoswimmers can be steered through non-Newtonian media exploiting their interaction with the fine structure of the surrounding media. For example, some screw propellers that have a filament diameter of about $70 \mathrm{~nm}$ have been shown to be capable of moving through high-viscosity solutions with velocities comparable to those of larger micropropellers, even though they are so small that Brownian forces suppress their actuation in pure water: when actuated in viscoelastic hyaluronan gels, these nanopropellers appear to have a significant advantage because they are of the same size range as the gel's mesh size (Schamel et al., 2014).

Swarming effects: Finally, collective effects in the interaction of several nanoswimmers can also lead to controllable directed motion due to short-range coupling of the motion of the individual entities (Saha, Golestanian, and Ramaswamy, 2014).

\section{OUTLOOK AND FUTURE DIRECTIONS}

Active particles have the potential to have far-reaching impact on many fields of science and technology. From the fundamental side, their study can shed light on the far-fromequilibrium physics underlying the adaptive and collective behavior of biological entities such as chemotactic bacteria and eukaryotic cells. From the more applied side, they provide options to perform tasks not easily achievable with other available techniques, such as the targeted localization, pickup, and delivery of microscopic and nanoscopic cargoes, e.g., in health care, environmental sustainability, and security.

However, there are still several open challenges that need to be tackled in order to achieve the full scientific and technological potential of microswimmers in real-life settings. In our opinion, the main scientific and technological challenges are the following:

(1) to understand more in depth and exploit their behavior in complex and crowded environments,

(2) to learn how to engineer emergent behaviors,

(3) to identify biocompatible propulsion mechanisms and energy supplies capable of lasting for the whole particle life cycle, and

(4) to scale down their dimensions toward the nanoscale. These challenges will necessarily take years or decades to be met and will need to involve concerted effort from academy and industry: in fact, although significant research progress has been accomplished over the past decade, the applicability of microswimmers to real-life problems is still in its infancy since current proof-of-concept research efforts on microswimmers and nanoswimmers need to be transformed into larger-scale pilot studies, and eventually into field applications. In all the future envisioned applications, in particular, understanding the physical interaction of microswimmers and nanoswimmers with their complex environment (e.g., tissues in living organisms, microchannels in lab-on-a-chip devices, obstacles and physical barriers in soils) represents an enabling step for the design of autonomous micromachines and nanomachines that can navigate efficiently in realistic conditions of operation.

Concretely, a series of so far unexplored (and potentially extremely interesting) research directions can be identifiedgoing from the most straightforward to the most imaginative as follows:

Addressing fundamental questions: Some fundamental problems are still open and need to be addressed in order for the field to be able to progress. These include, in particular, to understand how self-propelled Janus particles swim and interact, to explore under what conditions can thermodynamic equilibrium concepts be applied to detailed-balance-violating systems such as active matter, and to make synthetic microswimmers that will behave well at high densities avoiding problems with the formation of gas bubbles and fuel starvation.

Time-varying environments: Studying the behavior of microswimmers in time-varying potentials and complex environments is an interesting future direction of both fundamental and applied research.

Colloidal molecules: Formation of colloidal molecules between active particles (or passive particles in an active bath) with matching shapes is an interesting possibility. This has already been explored for the case of passive colloidal particles, e.g., the depletion-driven lock-and-key mechanism presented by Sacanna et al. (2010) and the self-assembly of tunable nanostructures using critical Casimir forces presented by Faber et al. (2013). The concept of passive colloidal molecules (Manoharan, Elsesser, and Pine, 2003; van Blaaderen, 2003; Kraft, Groenewold, and Kegel, 2009) was indeed recently generalized to active colloidal molecules. The latter comprise clusters of either diffusiophoretic particles (Soto and Golestanian, 2014, 2015) or tightly bound (Babel, Löwen, and Menzel, 2015) or magnetically attractive microswimmers (Kaiser, Popowa, and Löwen, 2015). In this context some new directions emerge from the possibility of using critical Casimir forces to fine-tune the particle assembly (Hertlein et al., 2008; Paladugu et al., 2016).

Hybrid systems: An interesting possibility is to use a combination of microfabricated and biological elements to generate motion on the nanoscale. For example, Gao et al. (2015) trapped smooth swimming bacteria in micron-sized structures and used their rotating flagella to generate a flow capable of transporting materials along designed trajectories.

Emergent collective organization: Albeit recently some studies have found interesting novel forms of aggregation in crowded suspensions of microswimmers (Buttinoni et al., 2013; Palacci et al., 2013), they still considered only one kind of microswimmer at a time. Nevertheless, we know from recent experiments in robotics (Halloy et al., 2007; Rubenstein, Cornejo, and Nagpal, 2014; Mijalkov et al., 2016; Volpe and Wehr, 2016) and biology (Gravish et al., 2015; Tennenbaum et al., 2016) that complex and robust collective 
behaviors can arise also in the presence of very simple constituent agents. The possibility of translating these results from robotic swarms to microswimmers and exploring how several different kinds of microswimmers can synergistically interact to perform a task is appealing.

Stochastic thermodynamics in active baths: Stochastic thermodynamics is an emergent field of research (Jarzynski, 2011; Seifert, 2012). Until now mostly systems coupled to a thermal bath and, therefore, satisfying Boltzmann statistics have been considered. However, systems satisfying these conditions are limited and, in particular, do not include many systems that are intrinsically far from equilibrium such as living matter, for which fluctuations are expected to be nonGaussian. For example, biomolecules within the cell are coupled with an active bath due to the presence of molecular motors within the cytoplasm, which leads to striking and largely not yet understood phenomena such as the emergence of anomalous diffusion (Barkai, Garini, and Metzler, 2012). Also, protein folding might be facilitated by the presence of active fluctuations (Harder, Valeriani, and Cacciuto, 2014) and active matter dynamics could play a central role in several biological functions (Mallory, Valeriani, and Cacciuto, 2015; Shin et al., 2015; Suzuki et al., 2015). It is therefore an open and compelling question to assess whether and to what degree stochastic thermodynamics can be applied to systems coupled to active baths (Argun et al., 2016).

Nanoswimmers: Active matter systems have so far been confined to microscopic (or larger) scales. However, with advances in creating artificial swimmers it could be possible to realize active matter systems on much smaller scales. In these regimes, thermal effects will be much more pronounced at room temperature and could mask the effects of the activity as seen in Sec. VI. However, experiments could be performed at lower temperatures and a new field of low-temperature active matter systems could be realized. At these smaller scales new effects may arise such as van der Waals forces, charging, or other fluctuation forces, which can lead to new types of active matter emergent behaviors. In terms of applications, activity on the nanoscale can be useful for creating new methods for self-assembly or pattern formation, changing the nature of fluids on the nanoscale, or serving as the first step to nanoscale probes and machines for medical applications.

Active polymers: The interaction of passive or active polymers with an active bath is a very interesting field. Open questions include how reptation dynamics might change when a chain is active rather than thermal, whether active polymer chains could produce unusual rheological properties, whether entanglement would increase or decrease under different activity levels, what would happen if the polymers were breakable, and whether glassy or jamming behavior can arise in dense active polymer mixtures. Extensions of such studies could be applied to understanding membranes, sheets, or flexible tubes in the presence of active matter baths.

Underdamped active particles: In most active matter systems the swimmers are considered to be moving in an overdamped medium. An alternative area of exploration is to consider cases of active systems where the damping is significantly reduced so that inertial effects can play an important role. One example might be to make active Janus particles that move through a dusty plasma (Morfill and Ivlev, 2009), air, or even a vacuum. In this context, an important class of systems is dusty plasma bilayers governed by effective nonreciprocal wake forces (Ivlev et al., 2015), which under appropriate conditions form self-propelled pairs (Bartnick et al., 2015). Another example is selfpropelling microdiodes (Chang et al., 2007; Sharma and Velev, 2015). Examples of questions that could be asked are whether activity-induced clustering transitions remain robust when inertial effects are present, or whether clustering can happen in a vacuum where dissipation occurs only at the particle-particle interaction level. When inertia is present, many nonlinear systems can exhibit a range of collective dynamical behaviors such as solitons, nonlinear waves, and shock phenomena (Infeld and Rowlands, 2000). If the medium is active, then new kinds of active soliton behaviors could occur, as well as new classes of selfpropagating waves or shocklike phenomena.

Swimmers in nonviscous fluids: Another question to ask is how artificial swimmers would behave in different kinds of fluids. Such effects could include shearthickening or shear-thinning fluids. It is also possible to explore anisotropic swimmers moving in a superfluid (Leggett, 1999), where new effects could arise due to the frictionless nature of the flow, as well as the generation of quantized vortex-antivortex couples when the velocity of the active particles is above the superfluid velocity (Bewley, Lathrop, and Sreenivasan, 2006). Possible experiments could be performed using swimmers in liquid helium (Bewley, Lathrop, and Sreenivasan, 2006) or in superfluids created in Bose-Einstein condensates (Madison et al., 2000).

Nonlocal interactions to mimic quantum effects: There have been a variety of efforts to create collective classical systems that mimic quantum phenomena (Couder and Fort, 2006; Bush, 2015). Another future direction for active matter systems is to explore the feasibility of making artificial swimmers that have effective nonlocal interactions generated via coupling or feedback responses, such as by using light fields or arrays of swimming robots with transmitters. For the correct choice of coupling rules, the active matter assembly could mimic the behavior of quantum systems. In this way swarms of swimmers could coordinate to form an effective wave-function-type object. 
On a more theoretical side it could be interesting to consider how activity would affect tunneling, interference, and entanglement.

Topological active matter systems on lattices: Recently there has been growing interest in topological effects in both quantum (Ran, Zhang, and Vishwanath, 2009; Hasan and Kane, 2010) and classical systems (Kane and Lubensky, 2014; Paulose, Chen, and Vitelli, 2015), where the behavior at the edge of the system is different from that in the bulk. Active particles moving on various kinds of finite lattices offer a system in which such topological effects could be investigated. For example, the activity could be enhanced at or occur only on the edges of the system. Additionally, in dense active matter systems higher-order topological objects such as dislocations could arise that could have dynamical properties that are significantly different from those of similar objects in purely thermal systems. For active matter on substrates, certain constraints could be imposed by the geometry of the substrate lattice that would allow only certain topological modes to propagate.

Active matter on active substrates: So far, active matter systems have been studied in the absence of a substrate or interacting with rigid substrates. However, in many real-world systems, particularly biological systems, the substrate is flexible rather than rigid, and in some cases the substrate itself is active. Thus, another future direction is understanding how active matter systems couple to flexible environments where the activity could in principle change the environment or cause some form of self-organization to occur. If the substrate itself is active, then some type of resonance could arise when the time scales of the substrate and the active matter match, and it is possible that biological active matter systems could harness the activity of a coupled system to enhance certain functions.

\section{ACKNOWLEDGMENTS}

We have greatly benefited from discussions with many colleagues and friends in the last few years. We especially thank Aykut Argun, Ivo Buttinoni, Agnese Callegari, Frank Cichos, Peer Fisher, Gerhard Gompper, Andreas Kaiser, Felix Kümmel, Mite Mijalkov, Roland Netz, Cynthia J. Olson-Reichhardt, Fernando Peruani, Roberto Piazza, Maurizio Righini, Holger Stark, Borge ten Hagen, Sabareeh K. P. Velu, Jan Wehr, Roland Winkler, and Raphael Wittkowski. C. B. and H. L. acknowledge funding from the SPP 1726 of the DFG. R.D.L. acknowledges funding from the European Research Council Grant Agreement No. 307940. G. V. acknowledges support from the Marie Curie Career Integration Grant (MC-CIG) No. PCIG11GA-2012-321726, a Distinguished Young Scientist award of the Turkish Academy of Sciences (TÜBA), and COST Actions No. MP1205, No. IC1208, and No. MP1305.

\section{REFERENCES}

Abdelmohsen, L. K. E. A., F. Peng, Y. Tu, and D. A. Wilson, 2014, "Micro- and nano-motors for biomedical applications," J. Mater. Chem. B 2, 2395-2408.

Ahuja, S., 2011, Ed., Chiral separation methods for pharmaceutical and biotechnological products (John Wiley \& Sons, New York).

Ai, B.-Q., Q.-Y. Chen, Y.-F. He, F.-G. Li, and W.-R. Zhong, 2013, "Rectification and diffusion of self-propelled particles in a two-dimensional corrugated channel," Phys. Rev. E 88, 062129.

Ai, B.-Q., Y.-F. He, and W.-R. Zhong, 2014, "Entropic ratchet transport of interacting active Brownian particles," J. Chem. Phys. 141, 194111.

Ai, B.-Q., Y.-F. He, and W.-R. Zhong, 2015, "Chirality separation of mixed chiral microswimmers in a periodic channel," Soft Matter 11, 3852-3859.

Ai, B.-Q., and J.-C. Wu, 2014, "Transport of active ellipsoidal particles in ratchet potentials," J. Chem. Phys. 140, 094103.

Ajdari, A., 2000, "Pumping liquids using asymmetric electrode arrays," Phys. Rev. E 61, R45.

Alexander, G.P., C. M. Pooley, and J. M. Yeomans, 2009, "Hydrodynamics of linked sphere model swimmers," J. Phys. Condens. Matter 21, 204108.

Alizadehrad, D., T. Krüger, M. Engstler, and H. Stark, 2015, "Simulating the complex cell design of trypanosoma brucei and its motility," PLoS Comput. Biol. 11, e1003967.

Angelani, L., A. Costanzo, and R. Di Leonardo, 2011, "Active ratchets," Europhys. Lett. 96, 68002.

Angelani, L., and R. Di Leonardo, 2010, "Geometrically biased random walks in bacteria-driven micro-shuttles," New J. Phys. 12, 113017.

Angelani, L., R. Di Leonardo, and G. Ruocco, 2009, "Self-starting micromotors in a bacterial bath," Phys. Rev. Lett. 102, 048104.

Angelani, L., C. Maggi, M. L. Bernardini, A. Rizzo, and R. Di Leonardo, 2011, "Effective interactions between colloidal particles suspended in a bath of swimming cells," Phys. Rev. Lett. 107, 138302.

Ao, X., P. K. Ghosh, Y. Li, G. Schmid, P. Hänggi, and F. Marchesoni, 2014, "Active Brownian motion in a narrow channel," Eur. Phys. J. Spec. Top. 223, 3227-3242.

Ao, X., P. K. Ghosh, Y. Li, G. Schmid, P. Hänggi, and F. Marchesoni, 2015, "Diffusion of chiral Janus particles in a sinusoidal channel," Europhys. Lett. 109, 10003.

Argun, A., A.-R. Moradi, E. Pince, G. B. Bagci, and G. Volpe, 2016, "Experimental evidence of the failure of Jarzynski equality in active baths," arXiv:1601.01123.

Asakura, S., and F. Oosawa, 1954, "On interaction between two bodies immersed in a solution of macromolecules," J. Chem. Phys. 22, 1255-1256.

Babel, S., H. Löwen, and A. M. Menzel, 2015, "Dynamics of a linear magnetic "microswimmer molecule'," arXiv:1511.03918.

Babel, S., B. ten Hagen, and H. Löwen, 2014, "Swimming path statistics of an active Brownian particle with time-dependent selfpropulsion,” J. Stat. Mech. P02011.

Babič, D., C. Schmitt, and C. Bechinger, 2005, "Colloids as model systems for problems in statistical physics," Chaos 15, 026114.

Barkai, E., Y. Garini, and R. Metzler, 2012, "Strange kinetics of single molecules in living cells," Phys. Today 65, 29-35.

Bartnick, J., A. Kaiser, H. Löwen, and A. Ivlev, 2015, "Emerging activity in bilayered dispersions with wake-mediated interactions," arXiv:1507.08962. 
Baskaran, A., and M. C. Marchetti, 2009, "Statistical mechanics and hydrodynamics of bacterial suspensions," Proc. Natl. Acad. Sci. U.S.A. 106, 15567-15572.

Behringer, H., and R. Eichhorn, 2011, "Hard-wall interactions in soft matter systems: Exact numerical treatment," Phys. Rev. E 83, 065701.

Behringer, H., and R. Eichhorn, 2012, "Brownian dynamics simulations with hard-body interactions: Spherical particles," J. Chem. Phys. 137, 164108.

Berdakin, I., Y. Jeyaram, V. V. Moshchalkov, L. Venken, S. Dierckx, S. J. Vanderleyden, A. V. Silhanek, C. A. Condat, and V. I. Marconi, 2013, "Influence of swimming strategy on microorganism separation by asymmetric obstacles," Phys. Rev. E 87, 052702.

Berg, H. C., 2003, "The rotary motor of bacterial flagella," Annu. Rev. Biochem. 72, 19-54.

Berg, H. C., 2004, E. coli in Motion (Springer-Verlag, Heidelberg, Germany).

Berg, H. C., and D. A. Brown, 1972, "Chemotaxis in Escherichia coli analysed by three-dimensional tracking," Nature (London) 239, 500-504.

Berg, H. C., and L. Turner, 1979, "Movement of microorganisms in viscous environments," Nature (London) 278, 349-351.

Berg, H. C., and L. Turner, 1990, "Chemotaxis of bacteria in glasscapillary arrays. Escherichia coli, motility, microchannel plate, and light-scattering," Biophys. J. 58, 919-930.

Berke, A.P., L. Turner, H.C. Berg, and E. Lauga, 2008, "Hydrodynamic attraction of swimming microorganisms by surfaces," Phys. Rev. Lett. 101, 038102.

Berthier, L., 2014, "Nonequilibrium glassy dynamics of selfpropelled hard disks," Phys. Rev. Lett. 112, 220602.

Berthier, L., H. Jacquin, and F. Zamponi, 2011, "Microscopic theory of the jamming transition of harmonic spheres," Phys. Rev. E 84, 051103.

Berthier, L., and J. Kurchan, 2013, "Non-equilibrium glass transitions in driven and active matter," Nat. Phys. 9, 310-314.

Bertin, E., M. Droz, and G. Grégoire, 2009, "Hydrodynamic equations for self-propelled particles: Microscopic derivation and stability analysis," J. Phys. A 42, 445001.

Bet, B., G. Boosten, M. Dijkstra, and R. van Roij, 2016, "Efficient shapes for microswimming: From three-body swimmers to helical flagella," arXiv:1603.02629.

Bewley, G. P., D. P. Lathrop, and K. R. Sreenivasan, 2006, "Superfluid helium: Visualization of quantized vortices," Nature (London) 441, 588-588.

Bialké, J., H. Löwen, and T. Speck, 2013, "Microscopic theory for the phase separation of self-propelled repulsive disks," Europhys. Lett. 103, 30008.

Bialké, J., T. Speck, and H. Löwen, 2012, "Crystallization in a dense suspension of self-propelled particles," Phys. Rev. Lett. 108, 168301.

Bialké, J., T. Speck, and H. Löwen, 2015, "Active colloidal suspensions: Clustering and phase behavior," J. Non-Cryst. Solids 407, 367-375.

Bianchi, S., F. Saglimbeni, A. Lepore, and R. Di Leonardo, 2015, "Polar features in the flagellar propulsion of E. coli bacteria," Phys. Rev. E 91, 062705.

Blake, J. R., 1971, "A spherical envelope approach to ciliary propulsion," J. Fluid Mech. 46, 199-208.

Blake, J. R., and M. A. Sleigh, 1974, "Mechanics of ciliary locomotion," Biol. Rev. Camb. Philos. Soc. 49, 85-125.

Brambilla, M., E. Ferrante, M. Birattari, and M. Dorigo, 2013, "Swarm robotics: A review from the swarm engineering perspective," Swarm Intelligence 7, 1-41 [http://citeseerx.ist.psu .edu/viewdoc/summary?doi=10.1.1.469.3725].
Bratanov, V., F. Jenko, and E. Frey, 2015, "New class of turbulence in active fluids," Proc. Natl. Acad. Sci. U.S.A. 112, 15048-15053.

Bregulla, A., H. Yang, and F. Cichos, 2013, "Individually tunable micromachines driven by laser induced self propelled thermophoresis," in Optics in the Life Sciences, p. JT2A.29.

Breier, R. E., R. L. B. Selinger, G. Ciccotti, S. Herminghaus, and M. G. Mazza, 2014, "Spontaneous chiral symmetry breaking in model bacterial suspensions," arXiv:1406.4423.

Bricard, A., J.-B. Caussin, D. Das, C. Savoie, V. Chikkadi, K. Shitara, O. Chepizhko, F. Peruani, D. Saintillan, and D. Bartolo, 2015, "Emergent vortices in populations of colloidal rollers," Nat. Commun. 6, 7470.

Bricard, A., J.-B. Caussin, N. Desreumaux, O. Dauchot, and D. Bartolo, 2013, "Emergence of macroscopic directed motion in populations of motile colloids," Nature (London) 503, 95-98.

Brokaw, C. J., 1958, "Chemotaxis of bracken spermatozoids-the role of bimalate ions," J. Exp. Biol. 35, 192-196 [http://jeb .biologists.org/content/35/1/192.abstract].

Brokaw, C. J., 1959, "Random and oriented movements of bracken spermatozoids," J. Cell. Comp. Physiol. 54, 95-101.

Brokaw, C.J., 1965, "Non-sinusoidal bending waves of sperm flagella," J. Exp. Biol. 43, 155-169 [http://jeb.biologists.org/ content/43/1/155].

Brown, A., and W. Poon, 2014, "Ionic effects in self-propelled Pt-coated Janus swimmers," Soft Matter 10, 4016-4027.

Buhl, J., D. J. T. Sumpter, I. D. Couzin, J. J. Hale, E. Despland, E. R. Miller, and S.J. Simpson, 2006, "From disorder to order in marching locusts," Science 312, 1402-1406.

Bush, J. W. M., 2015, "The new wave of pilot-wave theory," Phys. Today $\mathbf{6 8 , 4 7 - 5 3 .}$

Buttinoni, I., J. Bialké, F. Kümmel, H. Löwen, C. Bechinger, and T. Speck, 2013, "Dynamical clustering and phase separation in suspensions of self-propelled colloidal particles," Phys. Rev. Lett. 110, 238301.

Buttinoni, I., G. Volpe, F. Kümmel, G. Volpe, and C. Bechinger, 2012, "Active Brownian motion tunable by light," J. Phys. Condens. Matter 24, 284129.

Candelier, R., and O. Dauchot, 2009, "Creep motion of an intruder within a granular glass close to jamming," Phys. Rev. Lett. 103, 128001 .

Carbone, L., and P. D. Cozzoli, 2010, "Colloidal heterostructured nanocrystals: Synthesis and growth mechanisms," Nano Today 5, 449-493.

Carlsson, T., T. Ekholm, and C. Elvingson, 2010, "Algorithm for generating a Brownian motion on a sphere," J. Phys. A 43, 505001.

Casimir, H. B. G., 1948, "On the attraction between two perfectly conducting plates,” Proc. K. Ned. Akad. Wet. 51, 793-795 [https:// inspirehep.net/record/24990/].

Cates, M.E., 2012, "Diffusive transport without detailed balance: Does microbiology need statistical physics?” Rep. Prog. Phys. 75, 042601.

Cates, M. E., and J. Tailleur, 2013, "When are active Brownian particles and run-and-tumble particles equivalent? Consequences for motility induced phase separation," Europhys. Lett. 101, 20010.

Cates, M. E., and J. Tailleur, 2015, "Motility-induced phase separation," Annu. Rev. Condens. Matter Phys. 6, 219-244.

Cēbers, A., 2011, "Diffusion of magnetotactic bacterium in rotating magnetic field," J. Magn. Magn. Mater. 323, 279-282.

Chang, S. T., V. N. Paunov, D. N. Petsev, and O. D. Velev, 2007, "Remotely powered self-propelling particles and micropumps based on miniature diodes," Nat. Mater. 6, 235-240. 
Chaté, H., F. Ginelli, G. Grégoire, F. Peruani, and F. Raynaud, 2008a, "Modeling collective motion: Variations on the Vicsek model," Eur. Phys. J. B 64, 451-456.

Chaté, H., F. Ginelli, G. Grégoire, and F. Raynaud, 2008, "Collective motion of self-propelled particles interacting without cohesion," Phys. Rev. E 77, 046113.

Chattopadhyay, S., R. Moldovan, C. Yeung, and X. L. Wu, 2006, "Swimming efficiency of bacterium Escherichia coli," Proc. Natl. Acad. Sci. U.S.A. 103, 13712-13717.

Chen, H.-Y., and K.-T. Leung, 2006, "Rotating states of selfpropelling particles in two dimensions," Phys. Rev. E 73, 056107.

Chen, Q., and B.-Q. Ai, 2015, "Sorting of chiral active particles driven by rotary obstacles," J. Chem. Phys. 143, 104113.

Chen, X., X. Yang, M. Yang, and H. P. Zhang, 2015, "Dynamic clustering in suspension of motile bacteria," Europhys. Lett. 111, 54002.

Chen, Y.-F., S. Xiao, H.-Y. Chen, Y.-J. Sheng, and H.-K. Tsao, 2015, "Enhancing rectification of a nano-swimmer system by multilayered asymmetric barriers," Nanoscale 7, 16451-16459.

Chepizhko, O., E. G. Altmann, and F. Peruani, 2013, "Optimal noise maximizes collective motion in heterogeneous media," Phys. Rev. Lett. 110, 238101.

Chepizhko, O., and F. Peruani, 2013, "Diffusion, subdiffusion, and trapping of active particles in heterogeneous media," Phys. Rev. Lett. 111, 160604.

Chepizhko, O., and F. Peruani, 2015, "Active particles in heterogeneous media display new physics," Eur. Phys. J. Spec. Top. 224, 1287-1302.

Chilukuri, S., C. H. Collins, and P. T. Underhill, 2014, "Impact of external flow on the dynamics of swimming microorganisms near surfaces," J. Phys. Condens. Matter 26, 115101.

Chin, C. D., V. Linder, and S. K. Sia, 2007, "Lab-on-a-chip devices for global health: Past studies and future opportunities," Lab Chip 7, 41-57.

Cichocki, B., M. L. Ekiel-Jezewska, and E. Wajnryb, 2015, "Brownian motion of a particle with arbitrary shape," J. Chem. Phys. 142, 214902.

Cisneros, L. H., R. Cortez, C. Dombrowski, R. E. Goldstein, and J. O. Kessler, 2007, "Fluid dynamics of self-propelled microorganisms, from individuals to concentrated populations," Exp. Fluids 43, 737-753.

Contino, M., E. Lushi, I. Tuval, V. Kantsler, and M. Polin, 2015, "Microalgae scatter off solid surfaces by hydrodynamic and contact forces," Phys. Rev. Lett. 115, 258102.

Corkidi, G., B. Taboada, C. D. Wood, A. Guerrero, and A. Darszon, 2008, "Tracking sperm in three-dimensions," Biochem. Biophys. Res. Commun. 373, 125-129.

Costanzo, A., J. Elgeti, T. Auth, G. Gompper, and M. Ripoll, 2014, "Motility-sorting of self-propelled particles in microchannels," Europhys. Lett. 107, 36003.

Couder, Y., and E. Fort, 2006, "Single-particle diffraction and interference at a macroscopic scale," Phys. Rev. Lett. 97, 154101.

Coulais, C., R. P. Behringer, and O. Dauchot, 2014, "How the ideal jamming point illuminates the world of granular media," Soft Matter 10, 1519-1536.

Crenshaw, H. C., 1996, "A new look at locomotion in microorganisms: Rotating and translating," American Zoologist 36, 608-618.

Czirók, A., H.E. Stanley, and T. Vicsek, 1997, "Spontaneously ordered motion of self-propelled particles," J. Phys. A 30, 1375-1385.

Czirók, A., and T. Vicsek, 2000, "Collective behavior of interacting self-propelled particles," Physica A (Amsterdam) 281, 17-29.
Das, S. K., S. A. Egorov, B. Trefz, P. Virnau, and K. Binder, 2014, "Phase behavior of active swimmers in depletants: Molecular dynamics and integral equation theory," Phys. Rev. Lett. 112, 198301.

de Gennes, P. G., 1979, Scaling concepts in polymer physics (Cornell University Press, Ithaca, NY).

Derjaguin, B., and L. Landau, 1993, "Theory of the stability of strongly charged lyophobic sols and of the adhesion of strongly charged particles in solutions of electrolytes," Prog. Surf. Sci. 43, 30-59.

Deseigne, J., S. Leonard, O. Dauchot, and H. Chate, 2012, "Vibrated polar disks: Spontaneous motion, binary collisions, and collective dynamics," Soft Matter 8, 5629-5639.

Di Leonardo, R., L. Angelani, D. Dell'Arciprete, G. Ruocco, V. Iebba, S. Schippa, M. P. Conte, F. Mecarini, F. De Angelis, and E. Di Fabrizio, 2010, "Bacterial ratchet motors," Proc. Natl. Acad. Sci. U.S.A. 107, 9541-9545.

Di Leonardo, R., D. Dell'Arciprete, L. Angelani, and V. Iebba, 2011, "Swimming with an image," Phys. Rev. Lett. 106, 038101.

Di Leonardo, R., S. Keen, J. Leach, C. D. Saunter, G. D. Love, G. Ruocco, and M. Padgett, 2007, "Eigenmodes of a hydrodynamically coupled micron-size multiple-particle ring," Phys. Rev. E 76, 061402.

DiLuzio, W. R., L. Turner, M. Mayer, P. Garstecki, D. B. Weibel, H. C. Berg, and G. M. Whitesides, 2005, "Escherichia coli swim on the right-hand side," Nature (London) 435, 1271-1274.

Dombrowski, C., L. Cisneros, S. Chatkaew, R. E. Goldstein, and J. O. Kessler, 2004, "Self-concentration and large-scale coherence in bacterial dynamics," Phys. Rev. Lett. 93, 098103.

Downton, M. T., and H. Stark, 2009, "Simulation of a model microswimmer," J. Phys. Condens. Matter 21, 204101.

Drescher, K., J. Dunkel, L. H. Cisneros, S. Ganguly, and R. E. Goldstein, 2011, "Fluid dynamics and noise in bacterial cell-cell and cell-surface scattering," Proc. Natl. Acad. Sci. U.S.A. 108, 10940-10945.

Drescher, K., R. E. Goldstein, N. Michel, M. Polin, and I. Tuval, 2010, "Direct measurement of the flow field around swimming microorganisms," Phys. Rev. Lett. 105, 168101.

Dreyfus, R., J. Baudry, M. L. Roper, M. Fermigier, H. A. Stone, and J. Bibette, 2005, "Microscopic artificial swimmers," Nature (London) 437, 862-865.

Drocco, J. A., C. J. Olson-Reichhardt, and C. Reichhardt, 2012, "Bidirectional sorting of flocking particles in the presence of asymmetric barriers," Phys. Rev. E 85, 056102.

Dunkel, J., S. Heidenreich, K. Drescher, H. H. Wensink, M. Bär, and R. E. Goldstein, 2013, "Fluid dynamics of bacterial turbulence," Phys. Rev. Lett. 110, 228102.

Dusenbery, D. B., 1997, "Minimum size limit for useful locomotion by free-swimming microbes," Proc. Natl. Acad. Sci. U.S.A. 94, 10949-10954.

Ebbens, S. J., 2016, "Active colloids: Progress and challenges towards realising autonomous applications," Curr. Opin. Colloid Interface Sci. 21, 14-23.

Ebbens, S. J., and J. R. Howse, 2010, "In pursuit of propulsion at the nanoscale," Soft Matter 6, 726-738.

Ebel, J.P., J. L. Anderson, and D.C. Prieve, 1988, "Diffusiophoresis of latex particles in electrolyte gradients," Langmuir 4, 396-406.

Elgeti, J., and G. Gompper, 2009, "Self-propelled rods near surfaces,” Europhys. Lett. 85, 38002.

Elgeti, J., and G. Gompper, 2013, "Wall accumulation of selfpropelled spheres,” Europhys. Lett. 101, 48003. 
Elgeti, J., R. G. Winkler, and G. Gompper, 2015, "Physics of microswimmers: Single particle motion and collective behavior," Rep. Prog. Phys. 78, 056601.

Erdmann, U., W. Ebeling, L. Schimansky-Geier, and F. Schweitzer, 2000, “Brownian particles far from equilibrium," Eur. Phys. J. B 15, 105-113.

Erhardt, R., A. Böker, H. Zettl, H. Kaya, W. Pyckhout-Hintzen, G. Krausch, V. Abetz, and A. H. E. Müller, 2001, "Janus micelles," Macromolecules 34, 1069-1075.

Ermak, D. L., and J. A. McCammon, 1978, "Brownian dynamics with hydrodynamic interactions," J. Chem. Phys. 69, 1352-1360.

Espinosa-Garcia, J., E. Lauga, and R. Zenit, 2013, "Fluid elasticity increases the locomotion of flexible swimmers," Phys. Fluids 25, 031701.

Ezhilan, B., R. Alonso-Matilla, and D. Saintillan, 2015, "On the distribution and swim pressure of run-and-tumble particles in confinement," J. Fluid Mech. 781, R4.

Faber, S., Z. Hu, G. H. Wegdam, and P. Schall, 2013, "Controlling colloidal phase transitions with critical Casimir forces," Nat. Commun. 4, 1584

Farage, T. F. F., P. Krinninger, and J. M. Brader, 2015, "Effective interactions in active Brownian suspensions," Phys. Rev. E 91, 042310 .

Felderhof, B. U., 2006, “The swimming of animalcules," Phys. Fluids 18, 063101.

Felderhof, B. U., 2014a, "Collinear swimmer propelling a cargo sphere at low Reynolds number," Phys. Rev. E 90, 053013.

Felderhof, B. U., 2014b, "Swimming of an assembly of rigid spheres at low Reynolds number," Eur. Phys. J. E 37, 110.

Felderhof, B. U., and R. B. Jones, 2014, "Optimal translational swimming of a sphere at low Reynolds number," Phys. Rev. E 90, 023008

Fenchel, T., and N. Blackburn, 1999, "Motile chemosensory behaviour of phagotrophic protists: Mechanisms for and efficiency in congregating at food patches," Protist 150, 325-336.

Fernandes, M. X., and J. G. de la Torre, 2002, "Brownian dynamics simulation of rigid particles of arbitrary shape in external fields," Biophys. J. 83, 3039-3048.

Fily, Y., A. Baskaran, and M. F. Hagan, 2015, "Dynamics and density distribution of strongly confined noninteracting nonaligning selfpropelled particles in a nonconvex boundary," Phys. Rev. E 91, 012125.

Fily, Y., S. Henkes, and M. C. Marchetti, 2014, "Freezing and phase separation of self-propelled disks," Soft Matter 10, 2132-2140.

Fily, Y., and M. C. Marchetti, 2012, "Athermal phase separation of self-propelled particles with no alignment," Phys. Rev. Lett. 108, 235702.

Foffano, G., J. S. Lintuvuori, K. Stratford, M.E. Cates, and D. Marenduzzo, 2012, "Colloids in active fluids: Anomalous microrheology and negative drag," Phys. Rev. Lett. 109, 028103.

Fournier-Bidoz, S., A. C. Arsenault, I. Manners, and G. A. Ozin, 2005, "Synthetic self-propelled nanorotors," Chem. Commun. (Cambridge), 441-443.

Franke, K., and H. Gruler, 1990, "Galvanotaxis of human granulocytes: Electric field jump studies," Eur. Biophys. J. 18, 334-346.

Franosch, T., M. Grimm, M. Belushkin, F. M. Mor, G. Foffi, L. Forró, and S. Jeney, 2011, "Resonances arising from hydrodynamic memory in Brownian motion," Nature (London) 478, 85-88.

Friedrich, B. M., and F. Jülicher, 2008, "The stochastic dance of circling sperm cells: Sperm chemotaxis in the plane," New J. Phys. 10, 123025.

Friedrich, B. M., and F. Jülicher, 2009, "Steering chiral microswimmers along noisy helical paths," Phys. Rev. Lett. 103, 068102.
Frymier, P. D., R. M. Ford, H. C. Berg, and P. T. Cummings, 1995, "Three-dimensional tracking of motile bacteria near a solid planar surface," Proc. Natl. Acad. Sci. U.S.A. 92, 6195-6199.

Fu, H. C., C. W. Wolgemuth, and T. R. Powers, 2009, "Swimming speeds of filaments in nonlinearly viscoelastic fluids," Phys. Fluids 21, 033102.

Fung, Y.C., 1981, Biomechanics (Springer-Verlag, Heidelberg, Germany).

Galajda, P., J. Keymer, P. Chaikin, and R. Austin, 2007, “A wall of funnels concentrates swimming bacteria," J. Bacteriol. 189, 8704-8707.

Galajda, P., J. Keymer, J. Dalland, S. Park, S. Kou, and R. Austin, 2008, "Funnel ratchets in biology at low Reynolds number: Choanotaxis," J. Mod. Opt. 55, 3413-3422.

Gangwal, S., O. J. Cayre, M.Z. Bazant, and O. D. Velev, 2008, "Induced-charge electrophoresis of metallodielectric particles," Phys. Rev. Lett. 100, 058302.

Gao, W., and J. Wang, 2014, "The environmental impact of micro/ nanomachines: A review," ACS Nano 8, 3170-3180.

Gao, Z., H. Li, X. Chen, and H. P. Zhang, 2015, "Using confined bacteria as building blocks to generate fluid flow," Lab Chip 15, 4555-4562.

Gauger, E., and H. Stark, 2006, "Numerical study of a microscopic artificial swimmer," Phys. Rev. E 74, 021907.

Ghosh, A., and P. Fischer, 2009, "Controlled propulsion of artificial magnetic nanostructured propellers," Nano Lett. 9, 2243-2245.

Ghosh, P. K., Y. Li, G. Marchegiani, and F. Marchesoni, 2015, "Memory effects and active Brownian diffusion," J. Chem. Phys. 143, 211101.

Ghosh, P. K., V. R. Misko, F. Marchesoni, and F. Nori, 2013, "Self-propelled Janus particles in a ratchet: Numerical simulations," Phys. Rev. Lett. 110, 268301.

Ginelli, F., and H. Chaté, 2010, "Relevance of metric-free interactions in flocking phenomena," Phys. Rev. Lett. 105, 168103.

Ginot, F., I. Theurkauff, D. Levis, C. Ybert, L. Bocquet, L. Berthier, and C. Cottin-Bizonne, 2015, "Nonequilibrium equation of state in suspensions of active colloids," Phys. Rev. X 5, 011004.

Giomi, L., 2015, "Geometry and topology of turbulence in active nematics," Phys. Rev. X 5, 031003.

Golestanian, R., T. B. Liverpool, and A. Ajdari, 2005, "Propulsion of a molecular machine by asymmetric distribution of reaction products," Phys. Rev. Lett. 94, 220801.

Golestanian, R., T. B. Liverpool, and A. Ajdari, 2007, "Designing phoretic micro-and nano-swimmers," New J. Phys. 9, 126.

Golestanian, R., J.M. Yeomans, and N. Uchida, 2011, "Hydrodynamics synchronisation at low Reynolds number," Soft Matter 7, 3074.

Gompper, G., T. Ihle, D. M. Kroll, and R. G. Winkler, 2009, "Multi-particle collision dynamics: A particle-based mesoscale simulation approach to the hydrodynamics of complex fluids," Adv. Polym. Sci. 221, 1-87.

Gravish, N., G. Gold, A. Zangwill, M. A. D. Goodisman, and D. I. Goldman, 2015, "Glass-like dynamics in confined and congested ant traffic," Soft Matter 11, 6552-6561.

Grégoire, G., and H. Chaté, 2004, "Onset of collective and cohesive motion," Phys. Rev. Lett. 92, 025702.

Grégoire, G., H. Chaté, and Y. Tu, 2001, "Active and passive particles: Modeling beads in a bacterial bath," Phys. Rev. E 64, 011902.

Gu, H., Z. Yang, J. Gao, C. K. Chang, and B. Xu, 2005, "Heterodimers of nanoparticles: Formation at a liquid-liquid interface and particle-specific surface modification by functional molecules," J. Am. Chem. Soc. 127, 34-35. 
Guasto, J. S., K. A. Johnson, and J. P. Gollub, 2010, “Oscillatory flows induced by microorganisms swimming in two dimensions," Phys. Rev. Lett. 105, 168102.

Guidobaldi, A., Y. Jeyaram, I. Berdakin, V. V. Moshchalkov, C. A. Condat, V. I. Marconi, L. Giojalas, and A. V. Silhanek, 2014, "Geometrical guidance and trapping transition of human sperm cells," Phys. Rev. E 89, 032720.

Guzick, D. S., et al., 2001, "Sperm morphology, motility, and concentration in fertile and infertile men," New England J. Med. 345, 1388-1393.

Halloy, J., et al., 2007, "Social integration of robots into groups of cockroaches to control self-organized choices," Science 318, $1155-1158$.

Hänggi, P., and F. Marchesoni, 2009, “Artificial Brownian motors: Controlling transport on the nanoscale," Rev. Mod. Phys. 81, 387-442.

Happel, J., and H. Brenner, 1991, Low Reynolds number hydrodynamics: With special applications to particulate media (Kluwer Academic Publishers, Dordrecht, Netherlands), 2nd ed.

Harder, J., S. A. Mallory, C. Tung, C. Valeriani, and A. Cacciuto, 2014, "The role of particle shape in active depletion," J. Chem. Phys. 141, 194901.

Harder, J., C. Valeriani, and A. Cacciuto, 2014, "Activity-induced collapse and reexpansion of rigid polymers," Phys. Rev. E 90, 062312.

Hasan, M.Z., and C.L. Kane, 2010, "Colloquium: Topological insulators," Rev. Mod. Phys. 82, 3045-3068.

Hauser, M. J. B., and L. Schimansky-Geier, 2015, "Statistical physics of self-propelled particles," Eur. Phys. J. Spec. Top. 224, 1147-1150.

Helbing, D., 2001, "Traffic and related self-driven many-particle systems," Rev. Mod. Phys. 73, 1067-1141.

Henkes, S., Y. Fily, and M. C. Marchetti, 2011, "Active jamming: Self-propelled soft particles at high density," Phys. Rev. E 84, 040301.

Hennes, M., K. Wolff, and H. Stark, 2014, "Self-induced polar order of active Brownian particles in a harmonic trap," Phys. Rev. Lett. 112, 238104.

Hernandez-Ortiz, J.P., C. G. Stoltz, and M. D. Graham, 2005, "Transport and collective dynamics in suspensions of confined swimming particles," Phys. Rev. Lett. 95, 204501.

Hertlein, C., L. Helden, A. Gambassi, S. Dietrich, and C. Bechinger, 2008, "Direct measurement of critical Casimir forces," Nature (London) 451, 172-175.

Hill, J., O. Kalkanci, J.L. McMurry, and H. Koser, 2007, "Hydrodynamic surface interactions enable Escherichia coli to seek efficient routes to swim upstream," Phys. Rev. Lett. 98, 068101.

Hinz, D. F., A. Panchenko, T.-Y. Kim, and E. Fried, 2014, "Motility versus fluctuations in mixtures of self-motile and passive agents," Soft Matter 10, 9082-9089.

Honerkamp, J., 1993, Stochastic dynamical systems: Concepts, numerical methods, data analysis (John Wiley \& Sons, New York).

Howse, J. R., R. A. L. Jones, A. J. Ryan, T. Gough, R. Vafabakhsh, and R. Golestanian, 2007, "Self-motile colloidal particles: From directed propulsion to random walk," Phys. Rev. Lett. 99, 048102.

Huang, L. R., E. C. Cox, R. H. Austin, and J. C. Sturm, 2004, "Displacement continuous particle separation through deterministic lateral displacement," Science 304, 987-990.

Hulme, S. E., W. R. DiLuzio, S. S. Shevkoplyas, L. Turner, M. Mayer, H. C. Berg, and G. M. Whitesides, 2008, "Using ratchets and sorters to fractionate motile cells of Escherichia coli by length," Lab Chip 8, 1888-1895.

Infeld, E., and G. Rowlands, 2000, Nonlinear waves, solitons and chaos (Cambridge University Press, Cambridge, UK).

Intravaia, F., S. Koev, I. W. Jung, A. A. Talin, P. S. Davids, R. S. Decca, D. A. R. Dalvit, V. A. Aksyuk, and D. Lopez, 2013, "Strong Casimir force reduction through metallic surface nanostructuring," Nat. Commun. 4, 3515.

Isele-Holder, R. E., J. Elgeti, and G. Gompper, 2015, "Self-propelled worm-like filaments: Spontaneous spiral formation, structure, and dynamics," Soft Matter 11, 7181-7190.

Ishikawa, T., N. Yoshida, H. Ueno, M. Wiedeman, Y. Imai, and T. Yamaguchi, 2011, "Energy transport in a concentrated suspension of bacteria," Phys. Rev. Lett. 107, 028102.

Ismagilov, R. F., A. Schwartz, N. Bowden, and G. M. Whitesides, 2002, "Autonomous movement and self-assembly," Angew. Chem., Int. Ed. Engl. 41, 652-654.

Ivlev, A. V., J. Bartnick, M. Heinen, C.-R. Du, V. Nosenko, and H. Löwen, 2015, "Statistical mechanics where Newton's third law is broken," Phys. Rev. X 5, 011035.

Izri, Z., M. N. van der Linden, S. Michelin, and O. Dauchot, 2014, "Self-propulsion of pure water droplets by spontaneous Marangoni-stress-driven motion," Phys. Rev. Lett. 113, 248302.

Jarzynski, C., 2011, "Equalities and inequalities: Irreversibility and the second law of thermodynamics at the nanoscale," Annu. Rev. Condens. Matter Phys. 2, 329-351.

Jékely, G., J. Colombelli, H. Hausen, K. Guy, E. Stelzer, F. Nédélec, and D. Arendt, 2008, "Mechanism of phototaxis in marine zooplankton," Nature (London) 456, 395-399.

Jennings, H.S., 1901, "On the significance of the the spiral swimming of organisms," Amer. Soc. Natural. 35, 369-378.

Jiang, H.-R., H. Wada, N. Yoshinaga, and M. Sano, 2009, "Manipulation of colloids by a nonequilibrium depletion force in a temperature gradient," Phys. Rev. Lett. 102, 208301.

Jiang, H.-R., N. Yoshinaga, and M. Sano, 2010, "Active motion of a Janus particle by self-thermophoresis in a defocused laser beam," Phys. Rev. Lett. 105, 268302.

Jones, P. H., O. M. Maragò, and G. Volpe, 2015, Optical tweezers: Principles and applications (Cambridge University Press, Cambridge, UK).

Joseph, A., et al., 2016, "Active delivery to the brain by chemotaxis," bioRxiv 061325 .

Kaiser, A., S. Babel, B. ten Hagen, C. von Ferber, and H. Löwen, 2015, "How does a flexible chain of active particles swell?" J. Chem. Phys. 142, 124905.

Kaiser, A., and H. Löwen, 2014, "Unusual swelling of a polymer in a bacterial bath," J. Chem. Phys. 141, 044903.

Kaiser, A., A. Peshkov, A. Sokolov, B. ten Hagen, H. Löwen, and I. S. Aranson, 2014, "Transport powered by bacterial turbulence," Phys. Rev. Lett. 112, 158101.

Kaiser, A., K. Popowa, and H. Löwen, 2015, "Active dipole clusters: From helical motion to fission," Phys. Rev. E 92, 012301.

Kaiser, A., K. Popowa, H. H. Wensink, and H. Löwen, 2013, "Capturing self-propelled particles in a moving microwedge," Phys. Rev. E 88, 022311.

Kaiser, A., H. H. Wensink, and H. Löwen, 2012, "How to capture active particles," Phys. Rev. Lett. 108, 268307.

Kane, C. L., and T. C. Lubensky, 2014, "Topological boundary modes in isostatic lattices," Nat. Phys. 10, 39-45.

Kantsler, V., J. Dunkel, M. Polin, and R. E. Goldstein, 2013, "Ciliary contact interactions dominate surface scattering of swimming eukaryotes," Proc. Natl. Acad. Sci. U.S.A. 110, 1187-1192. 
Kapral, R., 2008, "Multiparticle collision dynamics: Simulation of complex systems on mesoscales," Adv. Chem. Phys. 140, 89-146.

Keber, F. C., E. Loiseau, T. Sanchez, S. J. DeCamp, L. Giomi, M. J. Bowick, M. C. Marchetti, Z. Dogic, and A. R. Bausch, 2014, "Topology and dynamics of active nematic vesicles," Science $\mathbf{3 4 5}$, 1135-1139.

Keim, N. C., M. Garcia, and P. E. Arratia, 2012, "Fluid elasticity can enable propulsion at low Reynolds number," Phys. Fluids 24, 081703.

Kim, S., and S. J. Karrila, 2005, Microhydrodynamics: Principles and selected applications (Dover, New York).

Kirchhoff, Th., H. Löwen, and R. Klein, 1996, "Dynamical correlations in suspensions of charged rodlike macromolecules," Phys. Rev. E 53, 5011-5022.

Kirkegaard, J. B., A. O. Marron, and R. E. Goldstein, 2016, "Motility of Colonial choanoflagellates and the statistics of aggregate random walkers," Phys. Rev. Lett. 116, 038102.

Kloeden, P. E., and R. A. Pearson, 1999, Numerical solution of stochastic differential equations (Springer-Verlag, Heidelberg, Germany).

Koumakis, N., A. Lepore, C. Maggi, and R. Di Leonardo, 2013, "Targeted delivery of colloids by swimming bacteria," Nat. Commun. 4, 2588.

Koumakis, N., C. Maggi, and R. Di Leonardo, 2014, "Directed transport of active particles over asymmetric energy barriers," Soft Matter 10, 5695-5701.

Kraft, D. J., J. Groenewold, and W. K. Kegel, 2009, "Colloidal molecules with well-controlled bond angles," Soft Matter 5, 3823-3826.

Kraft, D. J., R. Wittkowski, B. ten Hagen, K. V. Edmond, D. J. Pine, and H. Löwen, 2013, "Brownian motion and the hydrodynamic friction matrix for colloidal particles of complex shape," Phys. Rev. E 88, 050301.

Kreuter, C., U. Siems, P. Nielaba, P. Leiderer, and A. Erbe, 2013, "Transport phenomena and dynamics of externally and selfpropelled colloids in confined geometry," Eur. Phys. J. Spec. Top. 222, 2923-2939.

Kudrolli, A., G. Lumay, D. Volfson, and L. S. Tsimring, 2008, "Swarming and swirling in self-propelled polar granular rods," Phys. Rev. Lett. 100, 058001.

Kumar, N., S. Ramaswamy, and A. K. Sood, 2011, "Symmetry properties of the large-deviation function of the velocity of a selfpropelled polar particle," Phys. Rev. Lett. 106, 118001.

Kumar, N., H. Soni, S. Ramaswamy, and A. K. Sood, 2014, "Flocking at a distance in active granular matter," Nat. Commun. 5, 4688 .

Kümmel, F., P. Shabestari, C. Lozano, G. Volpe, and C. Bechinger, 2015, "Formation, compression and surface melting of colloidal clusters by active particles," Soft Matter 11, 6187-6191.

Kümmel, F., B. ten Hagen, R. Wittkowski, I. Buttinoni, R. Eichhorn, G. Volpe, H. Löwen, and C. Bechinger, 2013, "Circular motion of asymmetric self-propelling particles," Phys. Rev. Lett. 110, 198302

Lam, K.-D. N. T., M. Schindler, and O. Dauchot, 2015, "Selfpropelled hard disks: Implicit alignment and transition to collective motion," New J. Phys. 17, 113056.

Lamoreaux, S. K., 1997, "Demonstration of the Casimir force in the 0.6 to $6 \mu \mathrm{m}$ range," Phys. Rev. Lett. 78, 5-8.

Lattuada, M., and T. A. Hatton, 2011, "Synthesis, properties and applications of Janus nanoparticles," Nano Today 6, 286-308.

Lauga, E., 2007, "Propulsion in a viscoelastic fluid," Phys. Fluids 19, 083104.
Lauga, E., W. R. DiLuzio, G. M. Whitesides, and H. A. Stone, 2006, "Swimming in circles: Motion of bacteria near solid boundaries," Biophys. J. 90, 400-412.

Lauga, E., and R.E. Goldstein, 2012, "Dance of the microswimmers," Phys. Today 65, No. 9, 30-35.

Lauga, E., and T. R. Powers, 2009, "The hydrodynamics of swimming microorganisms," Rep. Prog. Phys. 72, 096601.

Leggett, A. J., 1999, Superfluidity (Springer-Verlag, Heidelberg, Germany).

Leoni, M., J. Kotar, B. Bassetti, P. Cicuta, and M. C. Lagomarsino, 2009, "A basic swimmer at low Reynolds number," Soft Matter 5, 472-476.

Leptos, K. C., J. S. Guasto, J. P. Gollub, A. I. Pesci, and R. E. Goldstein, 2009, "Dynamics of enhanced tracer diffusion in suspensions of swimming eukaryotic microorganisms," Phys. Rev. Lett. 103, 198103.

Leshansky, A. M., 2009, "Enhanced low-Reynolds-number propulsion in heterogeneous viscous environments," Phys. Rev. E 80, 051911 .

Li, G., and J. X. Tang, 2009, "Accumulation of microswimmers near a surface mediated by collision and rotational Brownian motion," Phys. Rev. Lett. 103, 078101.

Li, H., and H. P. Zhang, 2013, "Asymmetric gear rectifies random robot motion," Europhys. Lett. 102, 50007.

Li, J., V. V. Singh, S. Sattayasamitsathit, J. Orozco, K. Kaufmann, R. Dong, W. Gao, B. Jurado-Sanchez, Y. Fedorak, and J. Wang, 2014, "Water-driven micromotors for rapid photocatalytic degradation of biological and chemical warfare agents," ACS Nano 8, 11118-11125.

Li, Y., P. K. Ghosh, F. Marchesoni, and B. Li, 2014, "Manipulating chiral microswimmers in a channel," Phys. Rev. E 90, 062301.

Li, Y.-X., R. Lukeman, and L. Edelstein-Keshet, 2008, "Minimal mechanisms for school formation in self-propelled particles," Physica D (Amsterdam) 237, 699-720.

Liao, Q., G. Subramanian, M. P. DeLisa, D. L. Koch, and M. Wu, 2007, "Pair velocity correlations among swimming Escherichia coli bacteria are determined by force-quadrupole hydrodynamic interactions," Phys. Fluids 19, 061701.

Lighthill, J., 1976, "Flagellar hydrodynamics," SIAM Rev. 18, 161-230.

Lighthill, M. J., 1952, “On the squirming motion of nearly spherical deformable bodies through liquids at very small Reynolds numbers," Commun. Pure Appl. Math. 5, 109-118.

Lin, Z., J.-L. Thiffeault, and S. Childress, 2011, "Stirring by squirmers," J. Fluid Mech. 669, 167-177.

Liu, A. J., and S. R. Nagel, 2010, "The jamming transition and the marginally jammed solid," Annu. Rev. Condens. Matter Phys. 1, 347-369.

Liu, B., T. R. Powers, and K. S. Breuer, 2011, "Force-free swimming of a model helical flagellum in viscoelastic fluids," Proc. Natl. Acad. Sci. U.S.A. 108, 19516-19520.

Locatelli, E., F. Baldovin, E. Orlandini, and M. Pierno, 2015, "Active Brownian particles escaping a channel in single file," Phys. Rev. E 91, 022109.

Love, J.C., B.D. Gates, D. B. Wolfe, K.E. Paul, and G. M. Whitesides, 2002, "Fabrication and wetting properties of metallic half-shells with submicron diameters," Nano Lett. 2, 891-894.

Lukić, B., S. Jeney, C. Tischer, A. J. Kulik, L. Forró, and E.-L. Florin, 2005, "Direct observation of nondiffusive motion of a Brownian particle," Phys. Rev. Lett. 95, 160601.

Lushi, E., H. Wioland, and R.E. Goldstein, 2014, "Fluid flows created by swimming bacteria drive self-organization in confined suspensions," Proc. Natl. Acad. Sci. U.S.A. 111, 9733-9738. 
Ma, X., K. Hahn, and S. Sanchez, 2015, "Catalytic mesoporous Janus nanomotors for active cargo delivery," J. Am. Chem. Soc. 137, 4976-4979.

Ma, X., A. Jannasch, U.-R. Albrecht, K. Hahn, A. Miguel-López, E. Schäffer, and S. Sánchez, 2015, "Enzyme-powered hollow mesoporous janus nanomotors," Nano Lett. 15, 7043-7050.

MacDonald, M.P., G.C. Spalding, and K. Dholakia, 2003, "Microfluidic sorting in an optical lattice," Nature (London) 426, 421-424.

Machemer, H., 1972, "Ciliary activity and the origin of metachrony in Paramecium: Effects of increased viscosity," J. Exp. Biol. 57, 239-259 [http://jeb.biologists.org/content/57/1/239].

Machta, B. B., S. L. Veatch, and J.P. Sethna, 2012, "Critical Casimir forces in cellular membranes," Phys. Rev. Lett. 109, 138101

Madison, K. W., F. Chevy, W. Wohlleben, and J. Dalibard, 2000, "Vortex formation in a stirred Bose-Einstein condensate," Phys. Rev. Lett. 84, 806-809.

Magariyama, Y., and S. Kudo, 2002, "A mathematical explanation of an increase in bacterial swimming speed with viscosity in linearpolymer solutions," Biophys. J. 83, 733-739.

Maggi, C., A. Lepore, J. Solari, A. Rizzo, and R. Di Leonardo, 2013, "Motility fractionation of bacteria by centrifugation," Soft Matter 9 , 10885-10890.

Maggi, C., U. M. B. Marconi, N. Gnan, and R. Di Leonardo, 2015, "Multidimensional stationary probability distribution for interacting active particles," Sci. Rep. 5, 10742.

Maggi, C., M. Paoluzzi, N. Pellicciotta, A. Lepore, L. Angelani, and R. Di Leonardo, 2014, "Generalized energy equipartition in harmonic oscillators driven by active baths," Phys. Rev. Lett. 113, 238303.

Maggi, C., F. Saglimbeni, M. Dipalo, F. De Angelis, and R. Di Leonardo, 2015, "Micromotors with asymmetric shape that efficiently convert light into work by thermocapillary effects," Nat. Commun. 6, 7855.

Maggi, C., J. Simmchen, F. Saglimbeni, J. Katuri, M. Dipalo, F. De Angelis, S. Sanchez, and R. Di Leonardo, 2016, "Self-assembly of micromachining systems powered by Janus micromotors," Small 12, 446-451.

Magiera, M. P., and L. Brendel, 2015, "Trapping of interacting propelled colloidal particles in inhomogeneous media," Phys. Rev. E 92, 012304.

Mahmud, G., C. J. Campbell, and B.A. Grzybowski, 2009, "Directing cell motions on micropatterned ratchets," Nat. Phys. 5, 606-612.

Makino, M., and M. Doi, 2004, "Brownian motion of a particle of general shape in Newtonian fluid," J. Phys. Soc. Jpn. 73, 2739-2745.

Mallory, S. A., A. Saric, C. Valeriani, and A. Cacciuto, 2014, "Anomalous thermomechanical properties of a self-propelled colloidal fluid," Phys. Rev. E 89, 052303.

Mallory, S. A., C. Valeriani, and A. Cacciuto, 2014, "Curvatureinduced activation of a passive tracer in an active bath," Phys. Rev. E 90, 032309.

Mallory, S. A., C. Valeriani, and A. Cacciuto, 2015, "Anomalous dynamics of an elastic membrane in an active fluid," Phys. Rev. E 92, 012314 .

Mano, N., and A. Heller, 2005, "Bioelectrochemical propulsion," J. Am. Chem. Soc. 127, 11574-11575.

Manoharan, V. N., M. T. Elsesser, and D. J. Pine, 2003, "Dense packing and symmetry in small clusters of microspheres," Science 301, 483-487.
Marchetti, M. C., J. F. Joanny, S. Ramaswamy, T. B. Liverpool, J. Prost, M. Rao, and R. A. Simha, 2013, "Hydrodynamics of soft active matter," Rev. Mod. Phys. 85, 1143-1188.

Marconi, U. M. B., N. Gnan, M. P. C. Maggi, and R. Di Leonardo, 2015, "Velocity distribution in active particles systems," arXiv:1512.04227.

Marconi, U. M. B., and C. Maggi, 2015, "Towards a statistical mechanical theory of active fluids," Soft Matter 11, 8768-8781.

Marcos, H. C. Fu., T. R. Powers, and R. Stocker, 2009, "Separation of microscale chiral objects by shear flow," Phys. Rev. Lett. 102, 158103.

Martens, K., L. Angelani, R. Di Leonardo, and L. Bocquet, 2012, "Probability distributions for the run-and-tumble bacterial dynamics: An analogy to the Lorentz model," Eur. Phys. J. E 35, 84.

Martinez, V. A., J. Schwarz-Linek, M. Reufer, L. G. Wilson, A. N. Morozov, and W. C. K. Poon, 2014, "Flagellated bacterial motility in polymer solutions," Proc. Natl. Acad. Sci. U.S.A. 111, 17771-17776.

Martinez-Pedrero, F., A. Ortiz-Ambriz, I. Pagonabarraga, and P. Tierno, 2015, "Colloidal microworms propelling via a cooperative hydrodynamic conveyor belt," Phys. Rev. Lett. 115, 138301.

Martinez-Pedrero, F., and P. Tierno, 2015, "Magnetic propulsion of self-assembled colloidal carpets: Efficient cargo transport via a conveyor-belt effect," Phys. Rev. Applied 3, 051003.

McCandlish, S. R., A. Baskaran, and M.F. Hagan, 2012, "Spontaneous segregation of self-propelled particles with different motilities," Soft Matter 8, 2527-2534.

McHenry, M. J., and J.A. Strother, 2003, "The kinematics of phototaxis in larvae of the ascidian Aplidium constellatum," Marine Biology 142, 173-184.

Mehandia, V., and R. N. Prabhu, 2008, "The collective dynamics of self-propelled particles,” J. Fluid Mech. 595, 239-64.

Meiners, J.-C., and S.R. Quake, 1999, "Direct measurement of hydrodynamic cross correlations between two particles in an external potential," Phys. Rev. Lett. 82, 2211-2214.

Mendelson, N. H., A. Bourque, K. Wilkening, K. R. Anderson, and J.C. Watkins, 1999, "Organized cell swimming motions in Bacillus subtilis colonies: Patterns of short-lived whirls and jets," J. Bacteriol. 181, 600-609 [http://jb.asm.org/content/181/2/600 .abstract].

Mijalkov, M., A. McDaniel, J. Wehr, and G. Volpe, 2016, "Engineering sensorial delay to control phototaxis and emergent collective behaviors," Phys. Rev. X 6, 011008.

Mijalkov, M., and G. Volpe, 2013, "Sorting of chiral microswimmers," Soft Matter 9, 6376-6381.

Miño, G., T. E. Mallouk, T. Darnige, M. Hoyos, J. Dauchet, J. Dunstan, R. Soto, Y. Wang, A. Rousselet, and E. Clement, 2011, "Enhanced diffusion due to active swimmers at a solid surface," Phys. Rev. Lett. 106, 048102.

Mognetti, B. M., A. Šarić, S. Angioletti-Uberti, A. Cacciuto, C. Valeriani, and D. Frenkel, 2013, "Living clusters and crystals from low-density suspensions of active colloids," Phys. Rev. Lett. 111, 245702.

Montenegro-Johnson, T. D., D. J. Smith, and D. Loghin, 2013, "Physics of rheologically enhanced propulsion: Different strokes in generalized Stokes," Phys. Fluids 25, 081903.

Morfill, G.E., and A. V. Ivlev, 2009, "Complex plasmas: An interdisciplinary research field," Rev. Mod. Phys. 81, 1353-1404.

Munday, J. N., F. Capasso, and V. A. Parsegian, 2009, "Measured long-range repulsive Casimir-Lifshitz forces," Nature (London) 457, 170-173. 
Najafi, A., and R. Golestanian, 2004, "Simple swimmer at low Reynolds number: Three linked spheres," Phys. Rev. E 69, 062901.

Nakata, S., Y. Iguchi, S. Ose, M. Kuboyama, T. Ishii, and K. Yoshikawa, 1997, "Self-rotation of a camphor scraping on water: New insight into the old problem," Langmuir 13, 4454-4458.

Narayan, V., S. Ramaswamy, and N. Menon, 2007, "Long-lived giant number fluctuations in a swarming granular nematic," Science $\mathbf{3 1 7}$, 105-108.

Nelson, B. J., I. K. Kaliakatsos, and J. J. Abbott, 2010, "Microrobots for minimally invasive medicine," Annu. Rev. Biomed. Eng. 12, $55-85$.

Nelson, B. J., and K.E. Peyer, 2014, "Micro- and nanorobots swimming in heterogeneous liquids," ACS Nano 8, 8718-8724.

Ni, R., M. A. Cohen Stuart, and P. G. Bolhuis, 2015, "Tunable long range forces mediated by self-propelled colloidal hard spheres," Phys. Rev. Lett. 114, 018302.

Ni, R., M. A. Cohen Stuart, and M. Dijkstra, 2013, "Pushing the glass transition towards random close packing using self-propelled hard spheres," Nat. Commun. 4, 2704.

Ni, R., M. A. Cohen Stuart, M. Dijkstra, and P. G. Bolhuis, 2014, "Crystallizing hard-sphere glasses by doping with active particles," Soft Matter 10, 6609-6613.

Nishiguchi, D., and M. Sano, 2015, "Mesoscopic turbulence and local order in Janus particles self-propelling under an ac electric field," Phys. Rev. E 92, 052309.

Nourhani, A., V. H. Crespi, and P. E. Lammert, 2015, "Guiding chiral self-propellers in a periodic potential," Phys. Rev. Lett. 115, 118101.

Obata, T., T. Ohyama, J. Shimada, H. Oshima, H. Hara, and S. Fujita, 2000, "Fluctuations in human's walking (ii)," AIP Conf. Proc. 519, 720.

Ohta, T., and T. Ohkuma, 2009, "Deformable self-propelled particles," Phys. Rev. Lett. 102, 154101.

Oliveira, C. L. N., A. P. Vieira, D. Helbing, J. S. Andrade, Jr., and H. J. Herrmann, 2016, "Keep-left behavior induced by asymmetrically profiled walls," Phys. Rev. X 6, 011003.

Olson-Reichhardt, C. J., and C. Reichhardt, 2010, "Fluctuations, jamming, and yielding for a driven probe particle in disordered disk assemblies," Phys. Rev. E 82, 051306.

Palacci, J., C. Cottin-Bizonne, C. Ybert, and L. Bocquet, 2010, "Sedimentation and effective temperature of active colloidal suspensions," Phys. Rev. Lett. 105, 088304.

Palacci, J., S. Sacanna, A. P. Steinberg, D. J. Pine, and P. M. Chaikin, 2013, "Living crystals of light-activated colloidal surfers," Science 339, 936-940.

Paladugu, S., A. Callegari, Y. Tuna, L. Barth, S. Dietrich, A. Gambassi, and G. Volpe, 2016, "Nonadditivity of critical Casimir forces," Nat. Commun. 7, 11403.

Paoluzzi, M., R. Di Leonardo, and L. Angelani, 2014, "Run-andtumble particles in speckle fields," J. Phys. Condens. Matter 26, 375101.

Parra-Rojas, C., and R. Soto, 2014, "Casimir effect in swimmer suspensions," Phys. Rev. E 90, 013024.

Patra, D., S. Sengupta, W. Duan, H. Zhang, R. Pavlick, and A. Sen, 2013, "Intelligent, self-powered, drug delivery systems," Nanoscale 5, 1273-1283.

Paulose, J., B. G. Chen, and V. Vitelli, 2015, "Topological modes bound to dislocations in mechanical metamaterials," Nat. Phys. 11, 153-156.

Paxton, W. F., P. T. Baker, T. R. Kline, Y. Wang, T. E. Mallouk, and A. Sen, 2006, "Catalytically induced electrokinetics for motors and micropumps," J. Am. Chem. Soc. 128, 14881-14888.
Paxton, W. F., K. C. Kistler, C. C. Olmeda, A. Sen, S. K. St. Angelo, Y. Cao, T.E. Mallouk, P. E. Lammert, and V.H. Crespi, 2004, "Catalytic nanomotors: Autonomous movement of striped nanorods," J. Am. Chem. Soc. 126, 13424-13431.

Peplow, M., 2015, "March of the machines," Nature (London) 525, 18-21.

Peruani, F., A. Deutsch, and M. Bär, 2006, "Nonequilibrium clustering of self-propelled rods," Phys. Rev. E 74, 030904.

Pesce, G., G. Volpe, O. M. Maragó, P. H. Jones, S. Gigan, A. Sasso, and G. Volpe, 2015, "Step-by-step guide to the realization of advanced optical tweezers," J. Opt. Soc. Am. B 32, B84-B98.

Pesce, G., G. Volpe, G. Volpe, and A. Sasso, 2014, "Long-term influence of fluid inertia on the diffusion of a Brownian particle," Phys. Rev. E 90, 042309.

Petroff, A. P., X.-L. Wu, and A. Libchaber, 2015, "Fast-moving bacteria self-organize into active two-dimensional crystals of rotating cells," Phys. Rev. Lett. 114, 158102.

Pinçe, E., S. K. P. Velu, A. Callegari, P. Elahi, S. Gigan, G. Volpe, and G. Volpe, 2016, "Disorder-mediated crowd control in an active matter system," Nat. Commun. 7, 10907.

Pohl, O., and H. Stark, 2014, "Dynamic clustering and chemotactic collapse of self-phoretic active particles," Phys. Rev. Lett. 112, 238303.

Pohl, O., and H. Stark, 2015, "Self-phoretic active particles interacting by diffusiophoresis: A numerical study of the collapsed state and dynamic clustering," Eur. Phys. J. E 38, 93.

Poon, W. C. K., 2013, in "From Clarkia to Escherichia and Janus: The physics of natural and synthetic active colloids," Proceedings of the International School of Physics "Enrico Fermi," Course CLXXXIV "Physics of Complex Colloid" (IOS Press, Amsterdam), pp. 317-386.

Potiguar, F. Q., G. A. Farias, and W. P. Ferreira, 2014, "Self-propelled particle transport in regular arrays of rigid asymmetric obstacles," Phys. Rev. E 90, 012307.

Pototsky, A., A. M. Hahn, and H. Stark, 2013, "Rectification of self-propelled particles by symmetric barriers," Phys. Rev. E 87, 042124.

Pozrikidis, C., 1992, Boundary integral and singularity methods for linearized viscous flow (Cambridge University Press, Cambridge, UK).

Purcell, E. M., 1977, “Life at low Reynolds number,” Am. J. Phys. 45, 3-11.

Pushkin, D. O., and J.M. Yeomans, 2013, "Fluid mixing by curved trajectories of microswimmers," Phys. Rev. Lett. 111, 188101

Pushkin, D. O., and J. M. Yeomans, 2014, "Stirring by swimmers in confined microenvironments," J. Stat. Mech. P04030.

Qiu, T., T. C. Lee, A. G. Mark, K. I. Morozov, R. Münster, O. Mierka, S. Turek, A. M. Leshansky, and P. Fischer, 2014, "Swimming by reciprocal motion at low Reynolds number," Nat. Commun. 5, 5119.

Quint, D. A., and A. Gopinathan, 2013, "Swarming in disordered environments," arXiv:1302.6564.

Ramaswamy, S., 2010, "The mechanics and statics of active matter," Annu. Rev. Condens. Matter Phys. 1, 323-345.

Ramos, A., H. Morgan, N. G. Green, and A. Castellanos, 1998, "Ac electrokinetics: A review of forces in microelectrode structures," J. Phys. D 31, 2338-2353.

Ran, Y., Y. Zhang, and A. Vishwanath, 2009, "One-dimensional topologically protected modes in topological insulators with lattice dislocations," Nat. Phys. 5, 298-303.

Ray, D., C. Reichhardt, and C. J. Olson Reichhardt, 2014, "Casimir effect in active matter systems," Phys. Rev. E 90, 013019. 
Redner, G. S., A. Baskaran, and M. F. Hagan, 2013, "Reentrant phase behavior in active colloids with attraction," Phys. Rev. E 88, 012305.

Redner, G. S., M. F. Hagan, and A. Baskaran, 2013, "Structure and dynamics of a phase-separating active colloidal fluid," Phys. Rev. Lett. 110, 055701.

Reichhardt, C., and C. J. Olson-Reichhardt, 2004, "Dynamic regimes and spontaneous symmetry breaking for driven colloids on triangular substrates," Europhys. Lett. 68, 303-309.

Reichhardt, C., and C.J. Olson-Reichhardt, 2011, "Dynamical freezing of active matter," Proc. Natl. Acad. Sci. U.S.A. 108, 19099-19100.

Reichhardt, C., and C. J. Olson-Reichhardt, 2013a, "Active matter ratchets with an external drift," Phys. Rev. E 88, 062310.

Reichhardt, C., and C. J. Olson-Reichhardt, 2013b, "Dynamics and separation of circularly moving particles in asymmetrically patterned arrays," Phys. Rev. E 88, 042306.

Reichhardt, C., and C. J. Olson-Reichhardt, 2014a, "Absorbing phase transitions and dynamic freezing in running active matter systems," Soft Matter 10, 7502-7510.

Reichhardt, C., and C. J. Olson-Reichhardt, 2014b, "Active matter transport and jamming on disordered landscapes," Phys. Rev. E 90, 012701.

Reichhardt, C., and C. J. Olson-Reichhardt, 2014c, "Aspects of jamming in two-dimensional athermal frictionless systems," Soft Matter 10, 2932-2944.

Reichhardt, C., and C. J. Olson-Reichhardt, 2015, "Active microrheology in active matter systems: Mobility, intermittency, and avalanches," Phys. Rev. E 91, 032313.

Reigh, S. Y., R. G. Winkler, and G. Gompper, 2012, "Synchronization and bundling of anchored bacterial flagella," Soft Matter 8, 4363-4372.

Reimann, P., 2002, "Brownian motors: Noisy transport far from equilibrium," Phys. Rep. 361, 57-265.

Restrepo-Pérez, L., L. Soler, C. Martínez-Cisneros, S. Sanchez, and O. G. Schmidt, 2014, "Trapping self-propelled micromotors with microfabricated chevron and heart-shaped chips," Lab Chip 14, 1515-1518.

Reynolds, C. W., 1987, "Flocks, herds and schools: A distributed behavioral model," ACM Siggraph Computer Graphics 21, 25-34.

Riedel, I. H., K. Kruse, and J. Howard, 2005, "A self-organized vortex array of hydrodynamically entrained sperm cells," Science 309, 300-303.

Rings, D., R. Schachoff, M. Selmke, F. Cichos, and K. Kroy, 2010, "Hot Brownian motion," Phys. Rev. Lett. 105, 090604.

Rodenborn, B., C.-H. Chen, H. L. Swinney, B. Liu, and H. P. Zhang, 2013, "Propulsion of microorganisms by a helical flagellum," Proc. Natl. Acad. Sci. U.S.A. 110, E338-E347.

Romanczuk, P., M. Bär, W. Ebeling, B. Lindner, and L. SchimanskyGeier, 2012, "Active Brownian particles," Eur. Phys. J. Spec. Top. 202, 1-162.

Rubenstein, M., A. Cornejo, and R. Nagpal, 2014, "Programmable self-assembly in a thousand-robot swarm," Science 345, 795-799.

Sacanna, S., W. T. M. Irvine, P. M. Chaikin, and D. J. Pine, 2010, "Lock and key colloids," Nature (London) 464, 575-578.

Saha, S., R. Golestanian, and S. Ramaswamy, 2014, "Clusters, asters, and collective oscillations in chemotactic colloids," Phys. Rev. E 89, 062316.

Saintillan, D., and M. J. Shelley, 2007, "Orientational order and instabilities in suspensions of self-locomoting rods," Phys. Rev. Lett. 99, 058102.
Samin, S., and R. van Roij, 2015, "Self-propulsion mechanism of active Janus particles in near-critical binary mixtures," Phys. Rev. Lett. 115, 188305.

Sanchez, S., A. N. Ananth, V. M. Fomin, M. Viehrig, and O. G. Schmidt, 2011, "Superfast motion of catalytic microjet engines at physiological temperature," J. Am. Chem. Soc. 133, 14860-14863.

Sanchez, T., D. T. N. Chen, S. J. DeCamp, M. Heymann, and Z. Dogic, 2012, "Spontaneous motion in hierarchically assembled active matter," Nature (London) 491, 431-434.

Sandoval, M., and L. Dagdug, 2014, "Effective diffusion of confined active Brownian swimmers," Phys. Rev. E 90, 062711.

Schaar, K., A. Zöttl, and H. Stark, 2015, "Detention times of microswimmers close to surfaces: Influence of hydrodynamic interactions and noise," Phys. Rev. Lett. 115, 038101.

Schaller, V., C. Weber, C. Semmrich, E. Frey, and A. R. Bausch, 2010, "Polar patterns of driven filaments," Nature (London) 467, $73-77$.

Schaller, V., C. A. Weber, B. Hammerich, E. Frey, and A. R. Bausch, 2011, "Frozen steady states in active systems," Proc. Natl. Acad. Sci. U.S.A. 108, 19183-19188.

Schamel, D., A. G. Mark, J. G. Gibbs, C. Miksch, K. I. Morozov, A. M. Leshansky, and P. Fischer, 2014, "Nanopropellers and their actuation in complex viscoelastic media," ACS Nano 8, 8794-8801.

Schirmacher, W., B. Fuchs, F. Höfling, and T. Franosch, 2015, "Anomalous magneto-transport in disordered structures: Classical edge-state percolation," Phys. Rev. Lett. 115, 240602.

Schmidt, S., J. van der Gucht, P. M. Biesheuvel, R. Weinkamer, E. Helfer, and A. Fery, 2008, "Non-Gaussian curvature distribution of actin-propelled biomimetic colloid trajectories," Eur. Biophys. J. 37, 1361-1366.

Schmitt, M., and H. Stark, 2013, "Swimming active droplet: A theoretical analysis," Europhys. Lett. 101, 44008.

Schneider, W. R., and R. N. Doetsch, 1974, "Effect of viscosity on bacterial motility," J. Bacteriol. 117, 696-701 [http://jb.asm.org/ content/117/2/696].

Schnitzer, M. J., 1993, "Theory of continuum random walks and application to chemotaxis," Phys. Rev. E 48, 2553-2568.

Schnitzer, M. J., S. M. Block, and H. C. Berg, 1990, "Strategies for chemotaxis," Biol. Chemotactic Response 46, 15-34.

Schwarz-Linek, J., C. Valeriani, A. Cacciuto, M. E. Cates, D. Marenduzzo, A. N. Morozov, and W. C. K. Poon, 2012, "Phase separation and rotor self-assembly in active particle suspensions," Proc. Natl. Acad. Sci. U.S.A. 109, 4052-4057.

Schweitzer, F., 2007, Brownian agents and active particles: Collective dynamics in the natural and social sciences (Springer-Verlag, Heidelberg, Germany).

Seifert, U., 2012, "Stochastic thermodynamics, fluctuation theorems and molecular machines," Rep. Prog. Phys. 75, 126001.

Sharma, R., and O.D. Velev, 2015, "Remote steering of selfpropelling microcircuits by modulated electric field," Adv. Funct. Mater. 25, 5512-5519.

Shenoy, V. B., D. T. Tambe, A. Prasad, and J. A. Theriot, 2007, "A kinematic description of the trajectories of listeria monocytogenes propelled by actin comet tails," Proc. Natl. Acad. Sci. U.S.A. 104, 8229-8234.

Shin, J., A. G. Cherstvy, W. K. Kim, and R. Metzler, 2015, "Facilitation of polymer looping and giant polymer diffusivity in crowded solutions of active particles," New J. Phys. 17, 113008 . 
Sipos, O., K. Nagy, R. Di Leonardo, and P. Galajda, 2015, "Hydrodynamic trapping of swimming bacteria by convex walls," Phys. Rev. Lett. 114, 258104.

Smallenburg, F., and H. Löwen, 2015, "Swim pressure on walls with curves and corners," Phys. Rev. E 92, 032304.

Snezhko, A., M. Belkin, I. S. Aranson, and W.-K. Kwok, 2009, "Self-assembled magnetic surface swimmers," Phys. Rev. Lett. 102, 118103.

Sokolov, A., M. M. Apodaca, B. A. Grzybowski, and I. S. Aranson, 2010, "Swimming bacteria power microscopic gears," Proc. Natl. Acad. Sci. U.S.A. 107, 969-974.

Sokolov, A., I. S. Aranson, J. O. Kessler, and R. E. Goldstein, 2007, "Concentration dependence of the collective dynamics of swimming bacteria," Phys. Rev. Lett. 98, 158102.

Solon, A. P., M. E. Cates, and J. Tailleur, 2015, "Active Brownian particles and run-and-tumble particles: A comparative study," Eur. Phys. J. Spec. Top. 224, 1231-1262.

Solon, A. P., H. Chaté, and J. Tailleur, 2015, "From phase to microphase separation in flocking models: The essential role of nonequilibrium fluctuations," Phys. Rev. Lett. 114, 068101.

Solon, A. P., Y. Fily, A. Baskaran, M. E. Cates, Y. Kafri, M. Kardar, and J. Tailleur, 2015, "Pressure is not a state function for generic active fluids," Nat. Phys. 11, 673-678.

Solon, A. P., J. Stenhammar, R. Wittkowski, M. Kardar, Y. Kafri, M. E. Cates, and J. Tailleur, 2015, "Pressure and phase equilibria in interacting active Brownian spheres," Phys. Rev. Lett. 114, 198301.

Solovev, A. A., Y. Mei, E. Bermúdez Ureña, G. Huang, and O. G. Schmidt, 2009, "Catalytic microtubular jet engines self-propelled by accumulated gas bubbles," Small 5, 1688-1692.

Solovev, A. A., S. Sanchez, M. Pumera, Y. F. Mei, and O. G. Schmidt, 2010, "Magnetic control of tubular catalytic microbots for the transport, assembly, and delivery of micro-objects," Adv. Funct. Mater. 20, 2430-2435.

Soto, R., and R. Golestanian, 2014, "Self-assembly of catalytically active colloidal molecules: Tailoring activity through surface chemistry," Phys. Rev. Lett. 112, 068301.

Soto, R., and R. Golestanian, 2015, "Self-assembly of active colloidal molecules with dynamic function," Phys. Rev. E 91, 052304.

Spagnolie, S. E., and E. Lauga, 2012, "Hydrodynamics of selfpropulsion near a boundary: Predictions and accuracy of far-field approximations," J. Fluid Mech. 700, 105-147.

Spagnolie, S. E., G. R. Moreno-Flores, D. Bartolo, and E. Lauga, 2015, "Geometric capture and escape of a microswimmer colliding with an obstacle," Soft Matter 11, 3396-3411.

Speck, T., J. Bialké, A. M. Menzel, and H. Löwen, 2014, "Effective Cahn-Hilliard equation for the phase separation of active Brownian particles," Phys. Rev. Lett. 112, 218304.

Squires, T. M., and J. F. Brady, 2005, "A simple paradigm for active and nonlinear microrheology," Phys. Fluids 17, 073101.

Stenhammar, J., R. Wittkowski, D. Marenduzzo, and M. E. Cates, 2015, "Activity-induced phase separation and self-assembly in mixtures of active and passive particles," Phys. Rev. Lett. 114, 018301.

Su, Y.-S., H.-C. Wang, and L. I, 2015, "Suppressing turbulence of self-propelling rods by strongly coupled passive particles," Phys. Rev. E 91, 030302.

Sumino, Y., K. H. Nagai, Y. Shitaka, D. Tanaka, K. Yoshikawa, H. Chaté, and K. Oiwa, 2012, "Large-scale vortex lattice emerging from collectively moving microtubules," Nature (London) $\mathbf{4 8 3}$, $448-452$.
Suzuki, R., C. A. Weber, E. Frey, and A. R. Bausch, 2015, "Polar pattern formation in driven filament systems requires non-binary particle collisions," Nat. Phys. 11, 839-843.

Tailleur, J., and M.E. Cates, 2008, "Statistical mechanics of interacting run-and-tumble bacteria," Phys. Rev. Lett. 100, 218103.

Tailleur, J., and M.E. Cates, 2009, "Sedimentation, trapping, and rectification of dilute bacteria," Europhys. Lett. 86, 60002.

Takagi, D., J. Palacci, A. B. Braunschweig, M. J. Shelley, and J. Zhang, 2014, "Hydrodynamic capture of microswimmers into sphere-bound orbits," Soft Matter 10, 1784-1789.

Takatori, S. C., and J.F. Brady, 2015, "A theory for the phase behavior of mixtures of active particles," Soft Matter 11, 7920-7931.

Takatori, S. C., W. Yan, and J. F. Brady, 2014, "Swim pressure: Stress generation in active matter," Phys. Rev. Lett. 113, 028103.

Taktikos, J., V. Zaburdaev, and H. Stark, 2011, "Modeling a selfpropelled autochemotactic walker," Phys. Rev. E 84, 041924.

ten Hagen, B., R. Wittkowski, and H. Löwen, 2011, "Brownian dynamics of a self-propelled particle in shear flow," Phys. Rev. E 84, 031105.

ten Hagen, B., R. Wittkowski, D. Takagi, F. Kümmel, C. Bechinger, and H. Löwen, 2015, "Can the self-propulsion of anisotropic swimmers be described by forces and torques?" J. Phys. Condens. Matter 27, 194110.

Tennenbaum, M., Z. Liu, D. Hu, and A. Fernandez-Nieves, 2016, "Mechanics of fire ant aggregations," Nat. Mater. 15, 54-59.

Teran, J., L. Fauci, and M. Shelley, 2010, "Viscoelastic fluid response can increase the speed and efficiency of a free swimmer," Phys. Rev. Lett. 104, 038101.

Thar, R., and T. Fenchel, 2001, "True chemotaxis in oxygen gradients of the sulfur-oxidizing bacterium Thiovulum majus," Appl. Environ. Microbiol. 67, 3299-3303.

Theurkauff, I., C. Cottin-Bizonne, J. Palacci, C. Ybert, and L. Bocquet, 2012, "Dynamic clustering in active colloidal suspensions with chemical signaling," Phys. Rev. Lett. 108, 268303.

Thiffeault, J.-L., and S. Childress, 2010, "Stirring by swimming bodies," Phys. Lett. A 374, 3487-3490.

Thüroff, F., C. A. Weber, and E. Frey, 2013, "Critical assessment of the Boltzmann approach to active systems," Phys. Rev. Lett. 111, 190601.

Thüroff, F., C. A. Weber, and E. Frey, 2014, "Numerical treatment of the Boltzmann equation for self-propelled particle systems," Phys. Rev. X 4, 041030.

Thutupalli, S., R. Seemann, and S. Herminghaus, 2011, "Swarming behavior of simple model squirmers," New J. Phys. 13, 073021.

Tierno, P., R. Golestanian, I. Pagonabarraga, and F. Sagués, 2008, "Controlled swimming in confined fluids of magnetically actuated colloidal rotors," Phys. Rev. Lett. 101, 218304.

Toner, J., Y. Tu, and S. Ramaswamy, 2005, "Hydrodynamics and phases of flocks," Ann. Phys. (Amsterdam) 318, 170-244.

Tung, C., J. Harder, C. Valeriani, and A. Cacciuto, 2016, "Microphase separation in two dimensional suspensions of self-propelled spheres and dumbbells," Soft Matter 12, 555-561.

Turner, L., W. S. Ryu, and H. C. Berg, 2000, "Real-time imaging of fluorescent flagellar filaments," J. Bacteriol. 182, 2793-2801.

Uhlenbeck, G. E., and L. S. Ornstein, 1930, "On the theory of the Brownian motion,” Phys. Rev. 36, 823-841.

Uspal, W.E., M.N. Popescu, S. Dietrich, and M. Tasinkevych, 2015a, "Rheotaxis of spherical active particles near a planar wall," Soft Matter 11, 6613-6632. 
Uspal, W. E., M.N. Popescu, S. Dietrich, and M. Tasinkevych, 2015 b, "Self-propulsion of a catalytically active particle near a planar wall: From reflection to sliding and hovering," Soft Matter 11, 434-438.

Valeriani, C., M. Li, J. Novosel, J. Arlt, and D. Marenduzzo, 2011, "Colloids in a bacterial bath: Simulations and experiments," Soft Matter 7, 5228-5238.

van Blaaderen, A., 2003, "Chemistry: Colloidal molecules and beyond," Science 301, 470-471.

van der Meer, B., L. Filion, and M. Dijkstra, 2016, "Fabricating large two-dimensional single colloidal crystals by doping with active particles," Soft Matter 12, 3406-3411.

Van Hecke, M., 2010, "Jamming of soft particles: Geometry, mechanics, scaling and isostaticity," J. Phys. Condens. Matter 22, 033101.

van Teeffelen, S., and H. Löwen, 2008, "Dynamics of a Brownian circle swimmer,” Phys. Rev. E 78, 020101.

van Teeffelen, S., U. Zimmermann, and H. Löwen, 2009, “Clockwisedirectional circle swimmer moves counterclockwise in Petri dishand ring-like confinements," Soft Matter 5, 4510-4519.

Verwey, E. J. W., 1947, "Theory of the stability of lyophobic colloids," J. Phys. Chem. 51, 631-636.

Vicsek, T., A. Czirók, E. Ben-Jacob, I. Cohen, and O. Shochet, 1995, "Novel type of phase transition in a system of self-driven particles," Phys. Rev. Lett. 75, 1226-1229.

Vicsek, T., and A. Zafeiris, 2012, "Collective motion," Phys. Rep. 517, 71-140.

Vigeant, M. A.-S., R. M. Ford, M. Wagner, and L. K. Tamm, 2002, "Reversible and irreversible adhesion of motile Escherichia coli cells analyzed by total internal reflection aqueous fluorescence microscopy," Appl. Environ. Microbiol. 68, 2794-2801.

Vilain, C., F. Goettmann, A. Moores, P. Le Floch, and C. Sanchez, 2007, "Study of metal nanoparticles stabilised by mixed ligand shell: A striking blue shift of the surface-plasmon band evidencing the formation of Janus nanoparticles," J. Mater. Chem. 17, 3509-3514.

Viswanathan, G. M., M. G. E. da Luz, E. P. Raposo, and H.E. Stanley, 2011, The physics of foraging: An introduction to random searches and biological encounters (Cambridge University Press, Cambridge, UK).

Vladescu, I. D., E. J. Marsden, J. Schwarz-Linek, V. A. Martinez, J. Arlt, A. N. Morozov, D. Marenduzzo, M. E. Cates, and W. C. K. Poon, 2014, "Filling an emulsion drop with motile bacteria," Phys. Rev. Lett. 113, 268101.

Volpe, G., I. Buttinoni, D. Vogt, H.-J. Kümmerer, and C. Bechinger, 2011, "Microswimmers in patterned environments," Soft Matter 7, 8810-8815.

Volpe, G., S. Gigan, and G. Volpe, 2014, "Simulation of the active Brownian motion of a microswimmer," Am. J. Phys. 82, 659-664.

Volpe, G., L. Kurz, A. Callegari, G. Volpe, and S. Gigan, 2014, "Speckle optical tweezers: Micromanipulation with random light fields," Opt. Express 22, 18159-18167.

Volpe, G., and G. Volpe, 2013, "Simulation of a Brownian particle in an optical trap," Am. J. Phys. 81, 224-230.

Volpe, G., G. Volpe, and S. Gigan, 2014, "Brownian motion in a speckle light field: Tunable anomalous diffusion and selective optical manipulation," Sci. Rep. 4, 3936.

Volpe, G., and J. Wehr, 2016, "Effective drifts in dynamical systems with multiplicative noise: A review of recent progress," Rep. Prog. Phys. 79, 053901.

Walther, A., and A. H. E. Müller, 2013, "Janus particles: Synthesis, self-assembly, physical properties, and applications," Chem. Rev. 113, 5194-5261.

Wan, M. B., and Y.-S. Jho, 2013, "Directed motion of elongated active polymers," Soft Matter 9, 3255-3261.
Wan, M. B., C. J. O. Reichhardt, Z. Nussinov, and C. Reichhardt, 2008, "Rectification of swimming bacteria and self-driven particle systems by arrays of asymmetric barriers," Phys. Rev. Lett. 101, 018102.

Wang, J., and W. Gao, 2012, "Nano/microscale motors: Biomedical opportunities and challenges," ACS Nano 6, 5745-5751.

Wang, W., L. A. Castro, M. Hoyos, and T.E. Mallouk, 2012, "Autonomous motion of metallic microrods propelled by ultrasound," ACS Nano 6, 6122-6132.

Wang, Z., H.-Y. Chen, Y.-J. Sheng, and H.-K. Tsao, 2014, "Diffusion, sedimentation equilibrium, and harmonic trapping of run-and-tumble nanoswimmers," Soft Matter 10, 3209-3217.

Wensink, H. H., J. Dunkel, S. Heidenreich, K. Drescher, R. E. Goldstein, H. Löwen, and J. M. Yeomans, 2012, "Meso-scale turbulence in living fluids,” Proc. Natl. Acad. Sci. U.S.A. 109, 14308-14313.

Wensink, H. H., V. Kantsler, R. E. Goldstein, and J. Dunkel, 2014, "Controlling active self-assembly through broken particle-shape symmetry,” Phys. Rev. E 89, 010302.

Wensink, H. H., and H. Löwen, 2008, "Aggregation of self-propelled colloidal rods near confining walls," Phys. Rev. E 78, 031409.

Wensink, H. H., and H. Löwen, 2012, "Emergent states in dense systems of active rods: From swarming to turbulence," J. Phys. Condens. Matter 24, 464130.

Wilson, D. A., R. J.M. Nolte, and J.C. M. van Hest, 2012, "Autonomous movement of platinum-loaded stomatocytes," Nat. Chem. 4, 268-274.

Wioland, H., F. G. Woodhouse, J. Dunkel, J. O. Kessler, and R. E. Goldstein, 2013, "Confinement stabilizes a bacterial suspension into a spiral vortex," Phys. Rev. Lett. 110, 268102.

Wittkowski, R., and H. Löwen, 2012, "Self-propelled Brownian spinning top: Dynamics of a biaxial swimmer at low Reynolds numbers," Phys. Rev. E 85, 021406.

Woolley, D. M., 2003, "Motility of spermatozoa at surfaces," Reproduction 126, 259-270.

Wu, J.-C., Q. Chen, and B.-Q. Ai, 2015, "Longitudinal rectification of chiral self-propelled particles induced by the transversal asymmetry," J. Stat. Mech. P07005.

Wu, X.-L., and A. Libchaber, 2000, "Particle diffusion in a quasi-two-dimensional bacterial bath," Phys. Rev. Lett. 84, 3017-3020.

Würger, A., 2015, "Self-diffusiophoresis of Janus particles in nearcritical mixtures," Phys. Rev. Lett. 115, 188304.

Wysocki, A., J. Elgeti, and G. Gompper, 2015, "Giant adsorption of microswimmers: Duality of shape asymmetry and wall curvature," Phys. Rev. E 91, 050302.

Yan, W., and J.F. Brady, 2015, "The force on a body in active matter," arXiv:1510.07731.

Yang, W., V. R. Misko, K. Nelissen, M. Kong, and F. M. Peeters, 2012, "Using self-driven microswimmers for particle separation," Soft Matter 8, 5175-5179.

Yang, X., M. L. Manning, and M. C. Marchetti, 2014, “Aggregation and segregation of confined active particles," Soft Matter 10, 6477-6484.

Yariv, E., and O. Schnitzer, 2014, "Ratcheting of Brownian swimmers in periodically corrugated channels: A reduced Fokker-Planck approach,” Phys. Rev. E 90, 032115.

Zheng, X., B. ten Hagen, A. Kaiser, M. Wu, H. Cui, Z. Silber-Li, and H. Löwen, 2013, "Non-Gaussian statistics for the motion of selfpropelled Janus particles: Experiment versus theory,' Phys. Rev. E 88, 032304.

Zöttl, A., and H. Stark, 2014, "Hydrodynamics determines collective motion and phase behavior of active colloids in quasi-twodimensional confinement," Phys. Rev. Lett. 112, 118101. 